\title{
A Cascade Reaction of Michael Addition and Truce-Smiles Rearrangement to
}

Synthesize Trisubstituted 4-Quinolone Derivatives

Caixia Xie ${ }^{\dagger, \ddagger}$, Di Yang ${ }^{\ddagger}$, Xinfeng Wang ${ }^{\ddagger}$, and Chen Ma*

†School of Chemistry and Chemical Engineering, Shandong University of Technology, Zibo 255049, P. R. China

${ }^{\ddagger}$ School of Chemistry and Chemical Engineering, Shandong University, Jinan 250100, P. R. China

Email: chenma@sdu.edu.cn (C.Ma); Tel.: +86 53188364464; Fax: +86 53188564464 ;

Table of Contents

1. X-ray Crystallography............................................ S2-S4

2. ${ }^{1} \mathrm{H}$ NMR, ${ }^{13} \mathrm{C}$ NMR and ${ }^{19}$ F NMR spectra.................................S5-S41 


\section{X-ray Crystallography}

Light yellow crystals of $3 \mathbf{a}\left(\mathrm{C}_{27} \mathrm{H}_{18} \mathrm{~N}_{2} \mathrm{O}_{3}\right)$ were crystallized from the solution by solvent evaporation after one week at ambient conditions. A suitable crystal was selected and mounted on a Rigaku Oxford Diffraction XtaLAB Synergy diffractometer coupled to a Rigaku Hypix detector with $\mathrm{Cu} \mathrm{K} \alpha$ radiation from PhotonJet micro-focus X-ray source. The crystal was kept at 293(2) K during data collection. The crystal structure was solved by Olex2 with the SHELXT structure solution program using Intrinsic Phasing and refined with the SHELXL refinement package using Least Squares minimisation. Crystallographic data and structure refinement for 3a is listed in Table S1, Table S2 and Table S3.

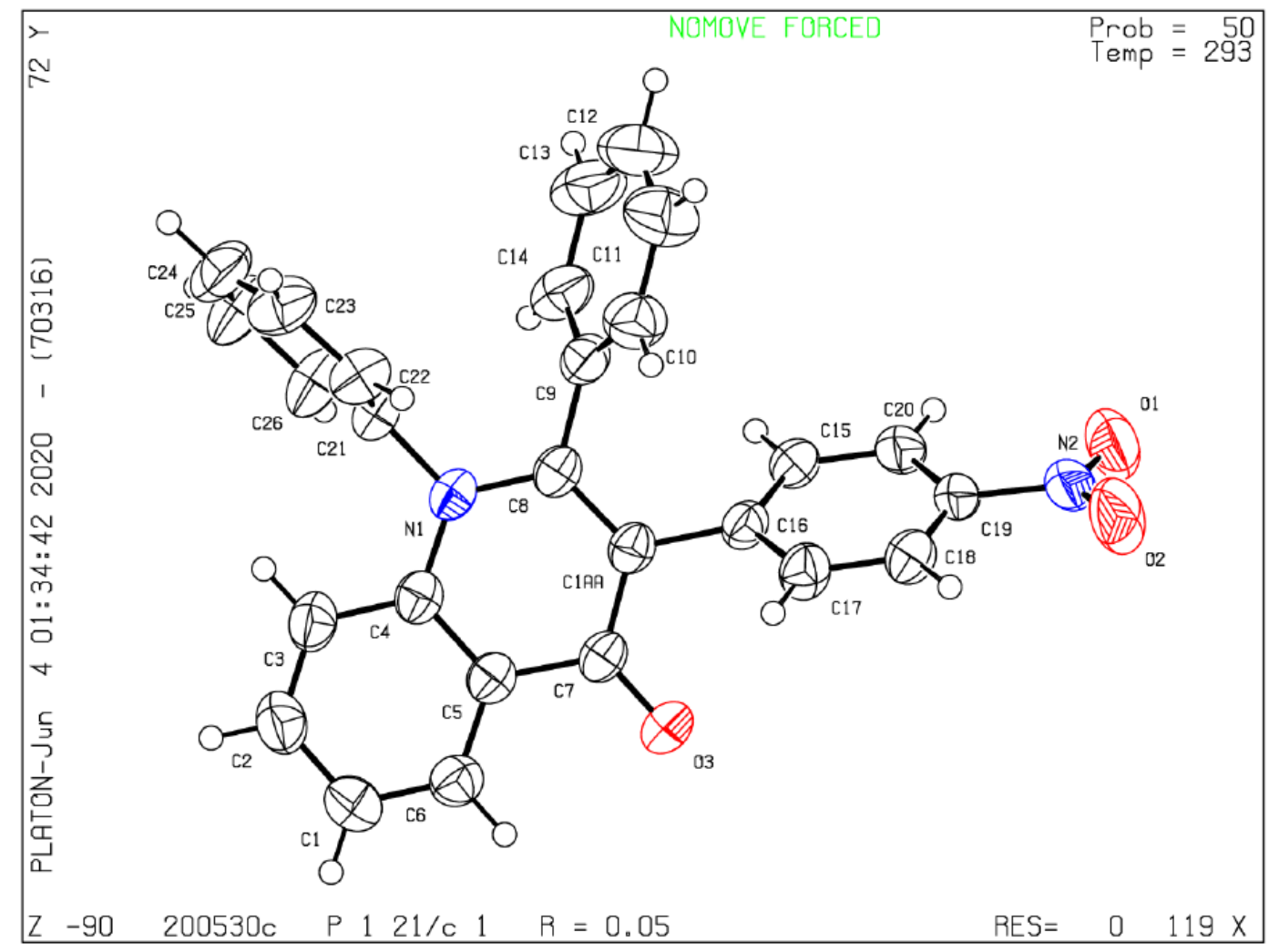

Figure S1. Asymmetric unit of 3a; thermal ellipsoids are drawn at the $\mathbf{5 0 \%}$ probability level.

Table S1 Crystal data and structure refinement for $3 a$.

\begin{tabular}{ll}
\hline Identification code & 3a \\
CCDC NO. & 2008744 \\
Empirical formula & $\mathrm{C}_{27} \mathrm{H}_{18} \mathrm{~N}_{2} \mathrm{O}_{3}$ \\
Formula weight & 418.43 \\
Temperature/K & $293(2)$
\end{tabular}




\begin{tabular}{|c|c|}
\hline Crystal system & monoclinic \\
\hline Space group & $\mathrm{P} 21 / \mathrm{c}$ \\
\hline $\mathrm{a} / \AA ̊$ & $8.9886(2)$ \\
\hline $\mathrm{b} / \AA$ & $23.6062(5)$ \\
\hline$c / \AA$ & $10.9049(2)$ \\
\hline$\alpha /^{\circ}$ & 90 \\
\hline$\beta /{ }^{\circ}$ & 113.831(3) \\
\hline$\gamma /{ }^{\circ}$ & 90 \\
\hline Volume $/ \AA^{3}$ & 2116.59(9) \\
\hline $\mathrm{Z}$ & 4 \\
\hline$\rho_{\text {calc }} \mathrm{g} / \mathrm{cm}^{3}$ & 1.313 \\
\hline$\mu / \mathrm{mm}^{-1}$ & 0.699 \\
\hline $\mathrm{F}(000)$ & 872.0 \\
\hline Crystal size/mm³ & $0.04 \times 0.04 \times 0.04$ \\
\hline Radiation & $\operatorname{CuK} \alpha(\lambda=1.54184)$ \\
\hline Reflections collected & 12544 \\
\hline Independent reflections & $4150\left[\mathrm{R}_{\text {int }}=0.0428, \mathrm{R}_{\text {sigma }}=0.0379\right]$ \\
\hline Data/restraints/parameters & $4150 / 0 / 290$ \\
\hline Goodness-of-fit on $\mathrm{F}^{2}$ & 1.142 \\
\hline Final $R$ indexes $[\mathrm{I}>=2 \sigma(\mathrm{I})]$ & $\mathrm{R}_{1}=0.0487, \mathrm{wR}_{2}=0.1325$ \\
\hline Final R indexes [all data] & $\mathrm{R}_{1}=0.0614, \mathrm{wR}_{2}=0.1370$ \\
\hline
\end{tabular}

Table S2. Bond Lengths for 3a.

\begin{tabular}{|c|c|c|c|c|c|}
\hline Atom & Atom & Length/Å & Atom & Atom & Length/Å \\
\hline $\mathrm{O} 3$ & C7 & $1.235(2)$ & C16 & C17 & $1.393(2)$ \\
\hline N1 & C8 & $1.378(2)$ & C15 & C20 & $1.380(3)$ \\
\hline N1 & C4 & $1.397(2)$ & C9 & C14 & $1.390(3)$ \\
\hline N1 & C21 & $1.453(2)$ & C9 & C10 & $1.384(3)$ \\
\hline $\mathrm{O} 1$ & N2 & $1.221(3)$ & C19 & C20 & $1.379(3)$ \\
\hline C1AA & C7 & $1.452(3)$ & C19 & C18 & $1.377(3)$ \\
\hline C1AA & C8 & $1.377(2)$ & C17 & C18 & $1.375(3)$ \\
\hline C1AA & C16 & $1.490(2)$ & C6 & C1 & $1.367(3)$ \\
\hline C7 & C5 & $1.465(3)$ & C22 & C23 & $1.380(3)$ \\
\hline N2 & $\mathrm{O} 2$ & $1.221(3)$ & C3 & $\mathrm{C} 2$ & $1.365(3)$ \\
\hline N2 & C19 & $1.464(3)$ & C14 & C13 & $1.382(4)$ \\
\hline C8 & C9 & $1.489(3)$ & C1 & C2 & $1.395(3)$ \\
\hline $\mathrm{C} 4$ & C5 & $1.400(2)$ & C26 & C25 & $1.388(3)$ \\
\hline $\mathrm{C} 4$ & C3 & $1.405(3)$ & C23 & C24 & $1.372(4)$ \\
\hline
\end{tabular}




\begin{tabular}{l|l|l|l|l|r|}
\hline C5 & C6 & $1.400(3)$ & C10 & C11 & $1.378(4)$ \\
\hline C21 & C22 & $1.372(3)$ & C24 & C25 & $1.366(4)$ \\
\hline C21 & C26 & $1.374(3)$ & C13 & C12 & $1.378(5)$ \\
\hline C16 & C15 & $1.389(3)$ & C11 & C12 & $1.381(5)$ \\
\hline
\end{tabular}

Table S3. Bond Angles for 3a.

\begin{tabular}{|c|c|c|c|c|c|c|c|}
\hline Atom & Atom & Atom & Angle ${ }^{\circ}$ & Atom & Atom & Atom & Angle $/^{\circ}$ \\
\hline C8 & N1 & C4 & 121.03(14) & C17 & C16 & C1AA & 118.81(16) \\
\hline $\mathrm{C} 8$ & N1 & $\mathrm{C} 21$ & $120.62(15)$ & $\mathrm{C} 20$ & C15 & C16 & $121.40(16)$ \\
\hline C4 & N1 & C21 & 117.32(15) & C14 & C9 & C8 & 121.15(19) \\
\hline C7 & C1AA & C16 & 116.43(15) & C10 & C9 & C8 & 119.63(18) \\
\hline $\mathrm{C} 8$ & C1AA & C7 & $120.96(16)$ & C10 & C9 & C14 & 119.2(2) \\
\hline C8 & C1AA & C16 & 122.61(16) & C20 & C19 & N2 & 119.19(18) \\
\hline $\mathrm{O} 3$ & C7 & C1AA & 123.23(17) & C18 & C19 & $\mathrm{N} 2$ & 118.88(17) \\
\hline $\mathrm{O} 3$ & C7 & C5 & 121.22(17) & C18 & C19 & $\mathrm{C} 20$ & 121.91(17) \\
\hline C1AA & C7 & $\mathrm{C} 5$ & $115.55(15)$ & C18 & C17 & C16 & 121.05(18) \\
\hline $\mathrm{O} 1$ & N2 & $\mathrm{O} 2$ & 123.1(2) & C19 & C20 & C15 & $118.35(18)$ \\
\hline $\mathrm{O} 1$ & N2 & C19 & 118.76(19) & $\mathrm{C} 1$ & C6 & C5 & 121.36(19) \\
\hline $\mathrm{O} 2$ & N2 & C19 & 118.1(2) & $\mathrm{C} 21$ & C22 & C23 & 119.5(2) \\
\hline N1 & C8 & C9 & $116.50(15)$ & $\mathrm{C} 2$ & C3 & C4 & $120.22(19)$ \\
\hline C1AA & $\mathrm{C} 8$ & N1 & 121.52(17) & C17 & C18 & C19 & 118.91(17) \\
\hline C1AA & $\mathrm{C} 8$ & C9 & 121.98(16) & C13 & C14 & C9 & $120.0(3)$ \\
\hline N1 & $\mathrm{C} 4$ & C5 & 119.52(16) & C6 & C1 & $\mathrm{C} 2$ & 119.1(2) \\
\hline N1 & $\mathrm{C} 4$ & $\mathrm{C} 3$ & 121.36(16) & C3 & $\mathrm{C} 2$ & C1 & 121.1(2) \\
\hline C5 & $\mathrm{C} 4$ & C3 & 119.11(18) & $\mathrm{C} 21$ & C26 & C25 & 118.9(2) \\
\hline $\mathrm{C} 4$ & C5 & C7 & 121.15(16) & $\mathrm{C} 24$ & C23 & C22 & $120.3(2)$ \\
\hline $\mathrm{C} 4$ & C5 & C6 & $119.09(17)$ & C11 & C10 & C9 & $120.8(3)$ \\
\hline C6 & C5 & C7 & 119.72(16) & $\mathrm{C} 25$ & C24 & C23 & 119.8(2) \\
\hline C22 & $\mathrm{C} 21$ & N1 & 119.18(16) & C24 & $\mathrm{C} 25$ & C26 & $120.6(2)$ \\
\hline $\mathrm{C} 22$ & $\mathrm{C} 21$ & $\mathrm{C} 26$ & 120.83(18) & C12 & C13 & C14 & 120.1(3) \\
\hline $\mathrm{C} 26$ & $\mathrm{C} 21$ & N1 & 119.78(17) & C10 & C11 & C12 & 119.6(3) \\
\hline C15 & C16 & C1AA & 122.73(15) & C13 & C12 & C11 & 120.3(3) \\
\hline C15 & C16 & C17 & $118.36(17)$ & & & & \\
\hline
\end{tabular}




\section{2. ${ }^{1} \mathrm{H}$ NMR and ${ }^{13} \mathrm{C}$ NMR spectra}

\section{3-(4-Nitrophenyl)-1,2-diphenylquinolin-4(1H)-one 3a}

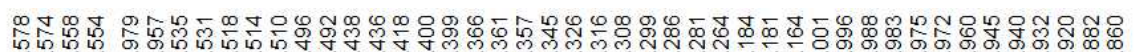
क

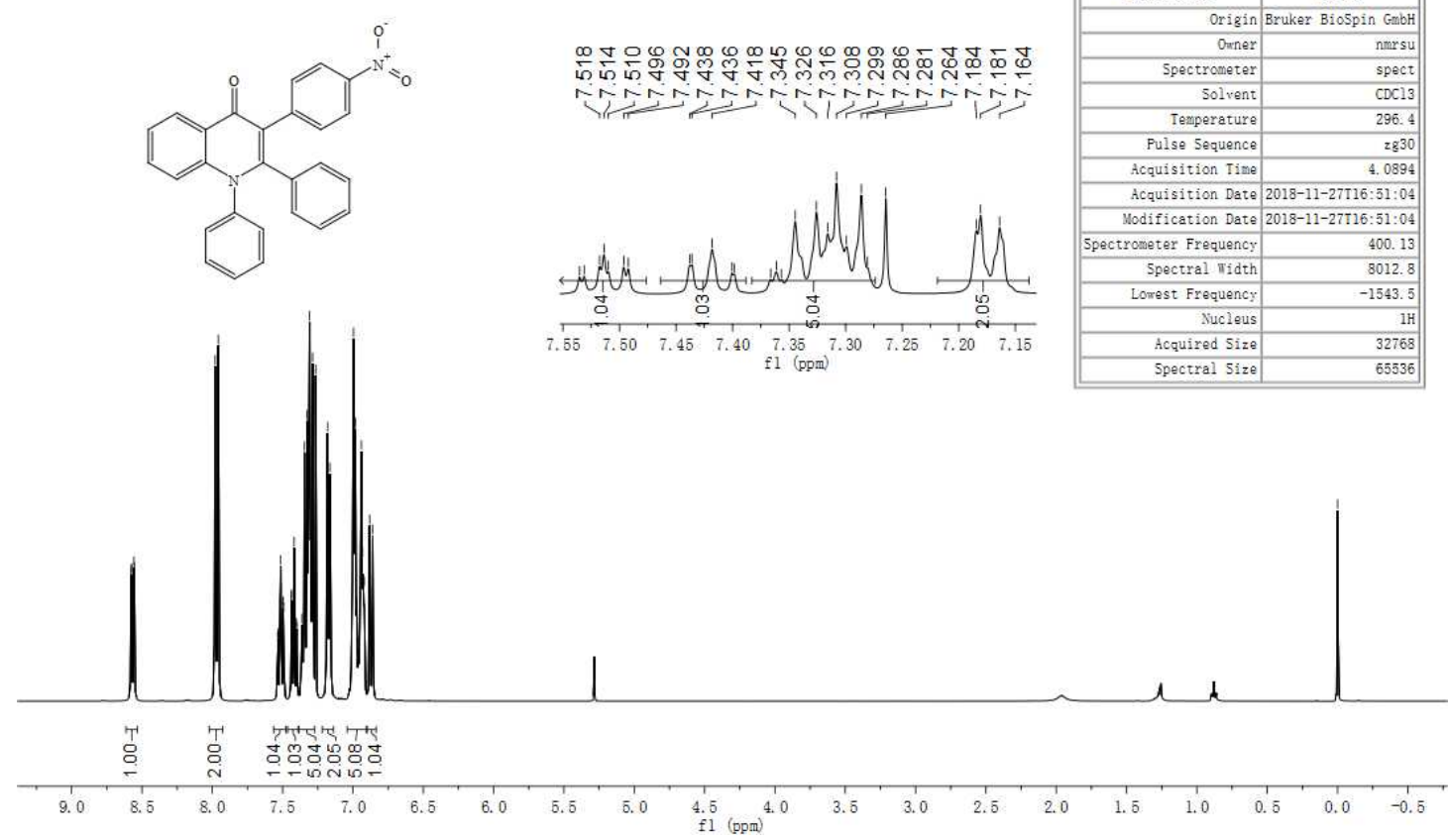

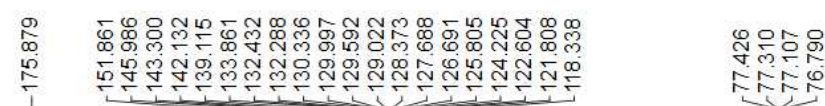
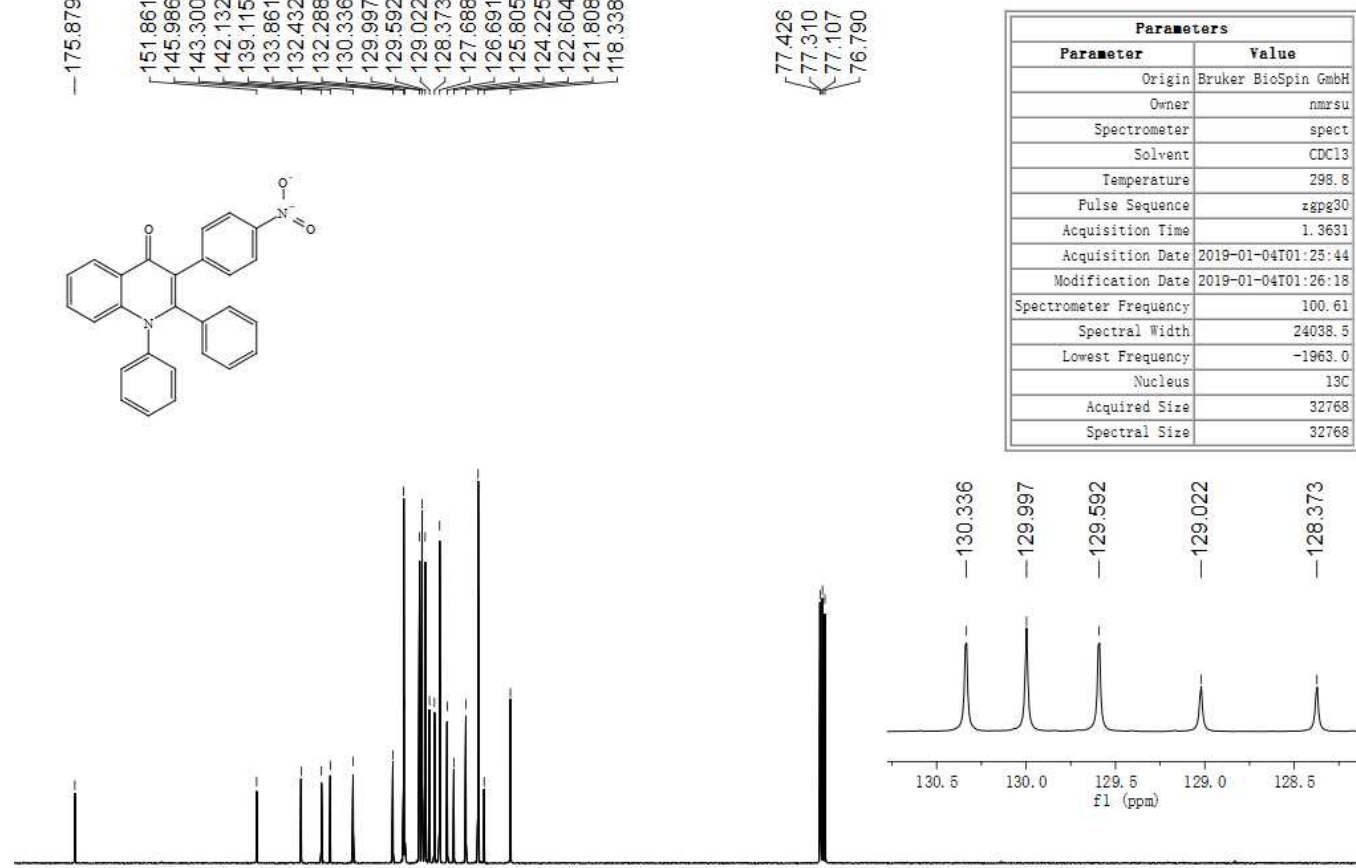

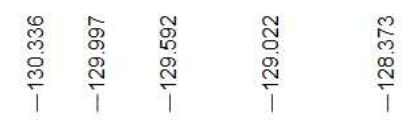
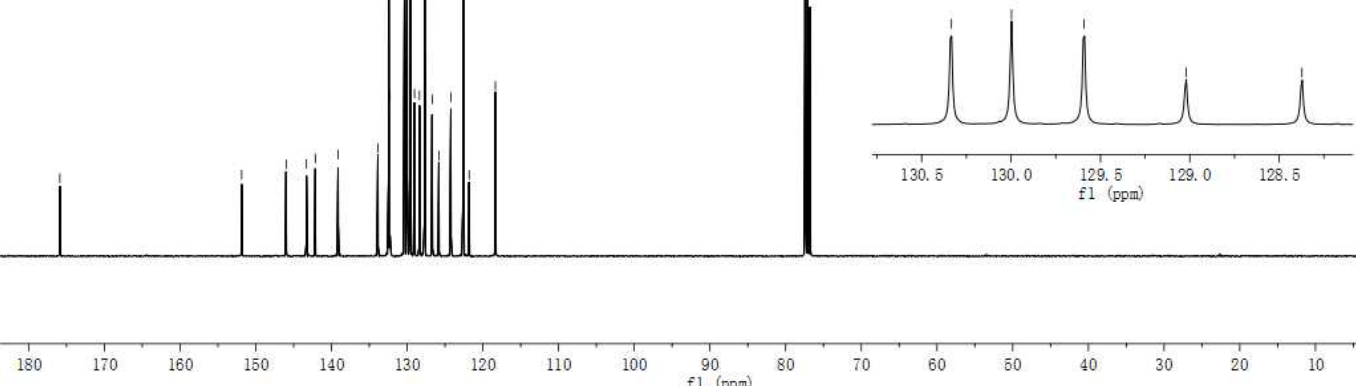
3-(4-Nitrophenyl)-2-phenyl-1-(p-tolyl)quinolin-4(1H)-one (3b)

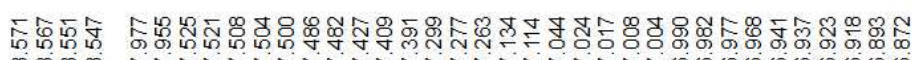

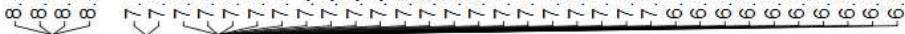

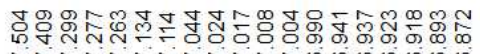

r.Nin
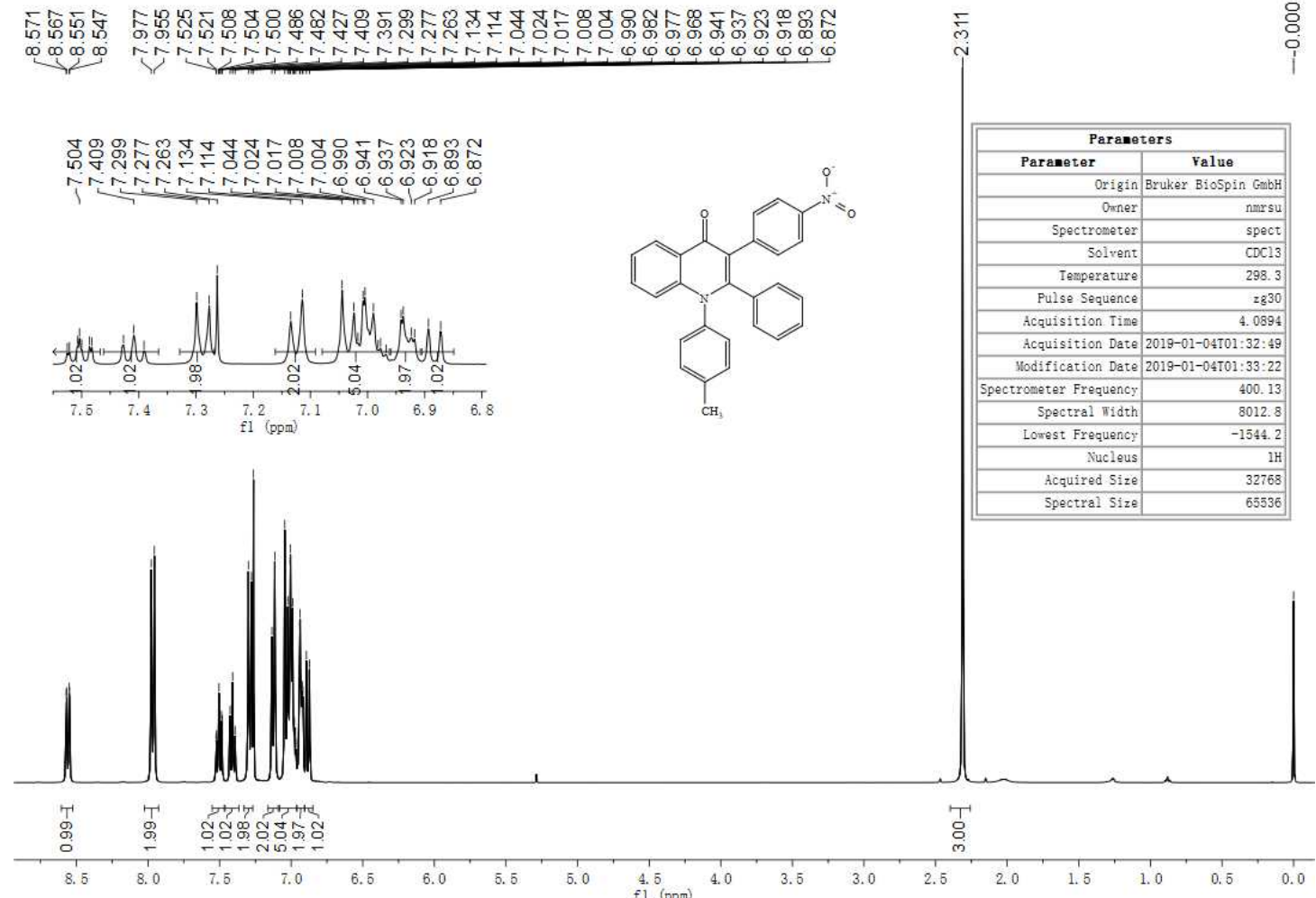

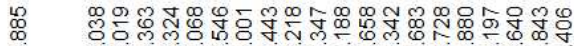

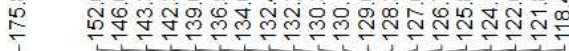

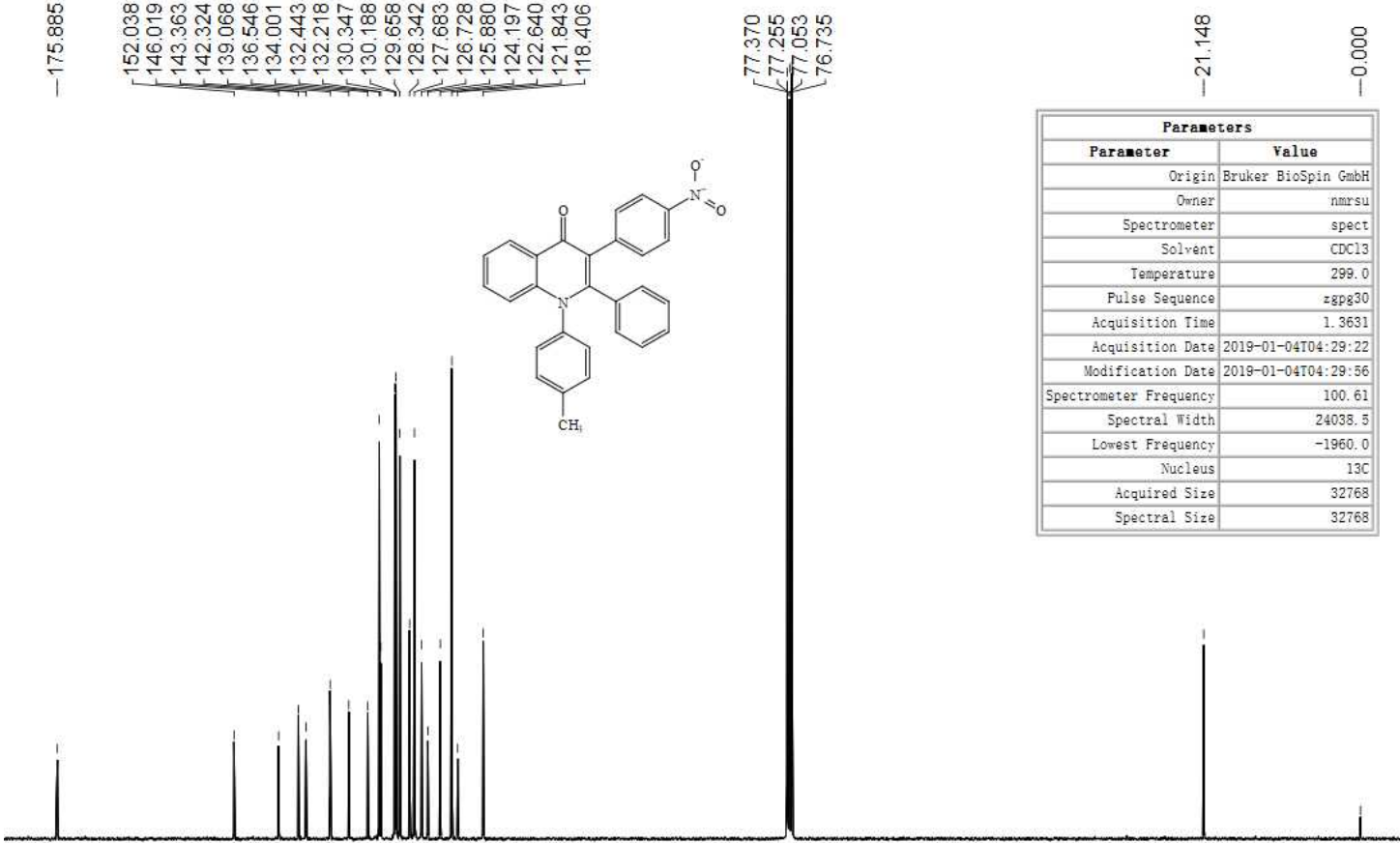


1-(4-Methoxyphenyl)-3-(4-nitrophenyl)-2-phenylquinolin-4(1H)-one (3c)

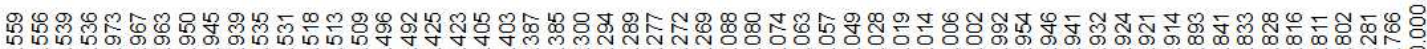

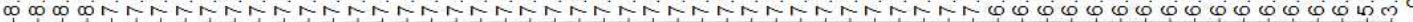
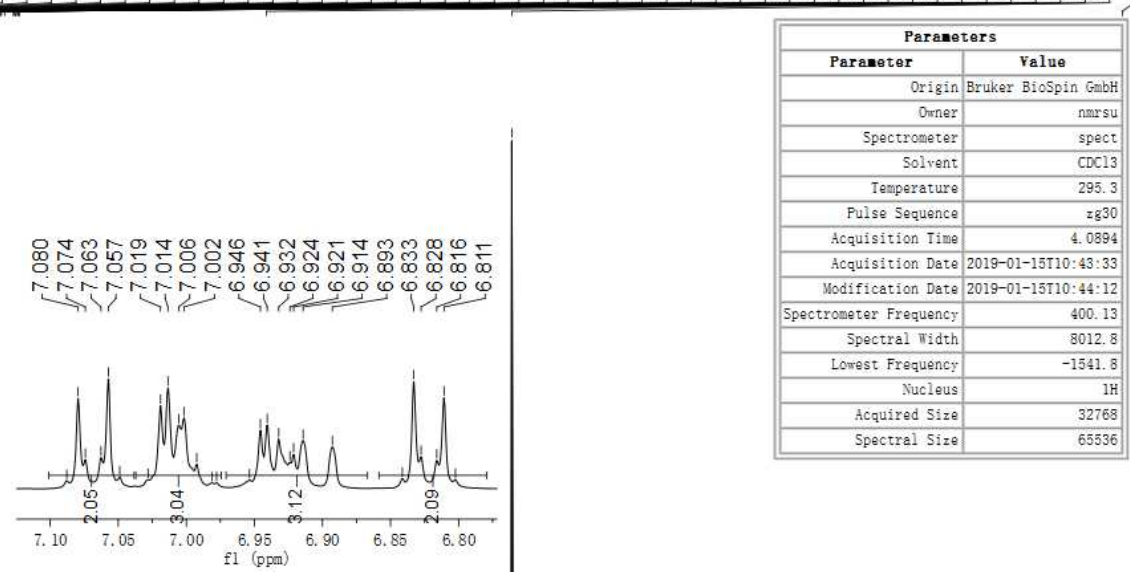

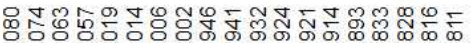
rininingen<smiles>CCN(CC)c1ccc(OC)cc1</smiles>

$$
\mid
$$

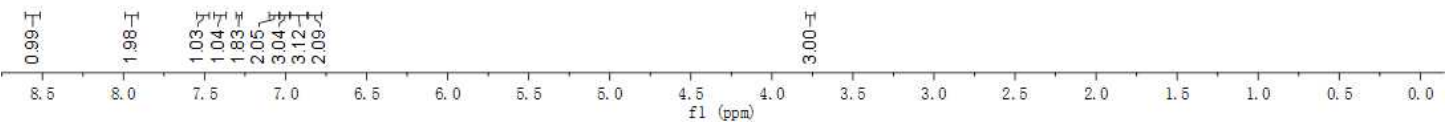
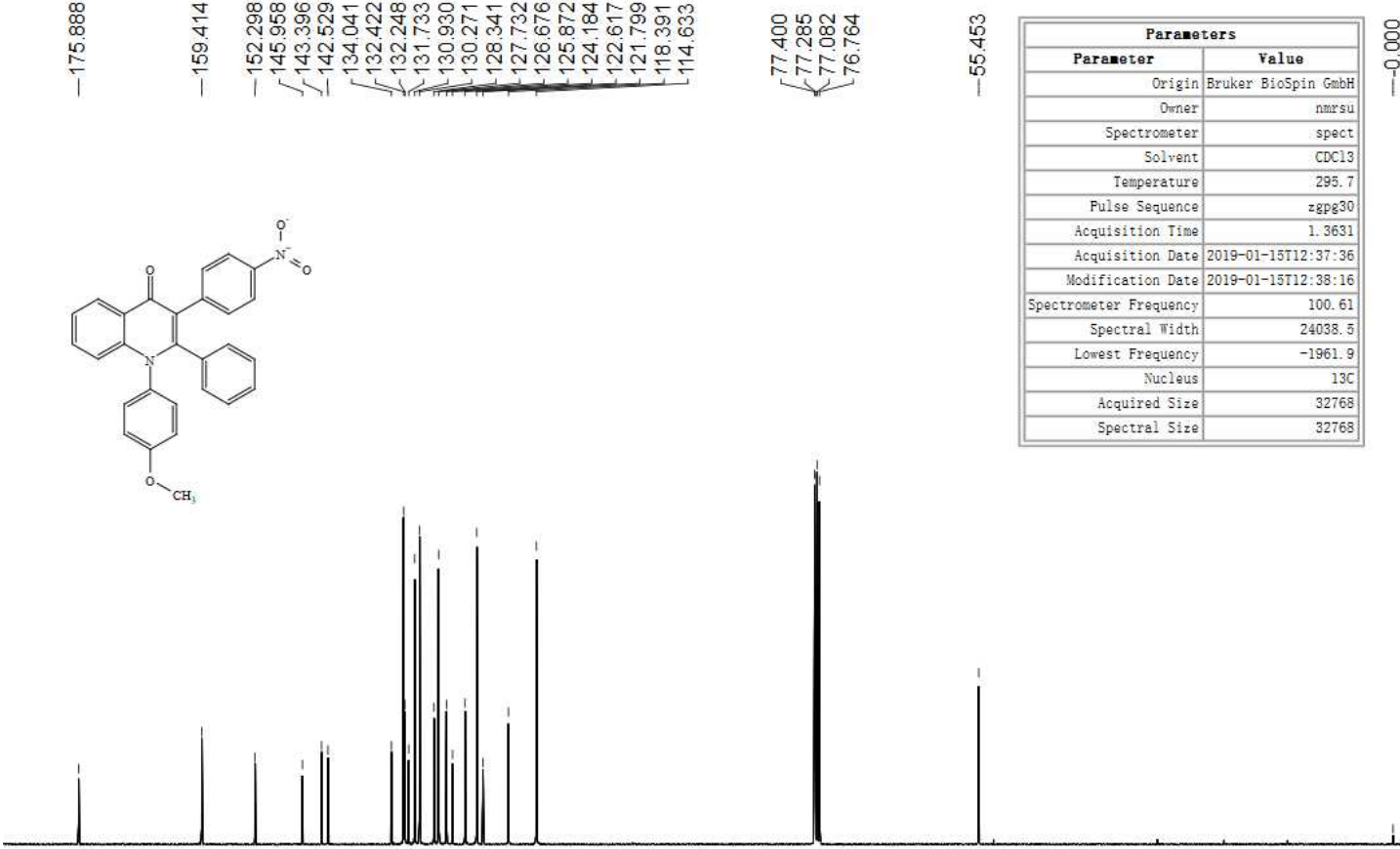

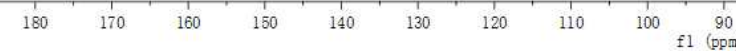


1-(4-Bromophenyl)-3-(4-nitrophenyl)-2-phenylquinolin-4(1H)-one (3d)

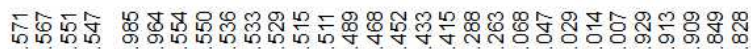

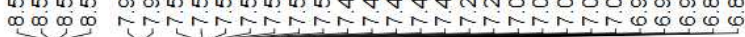

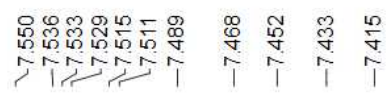

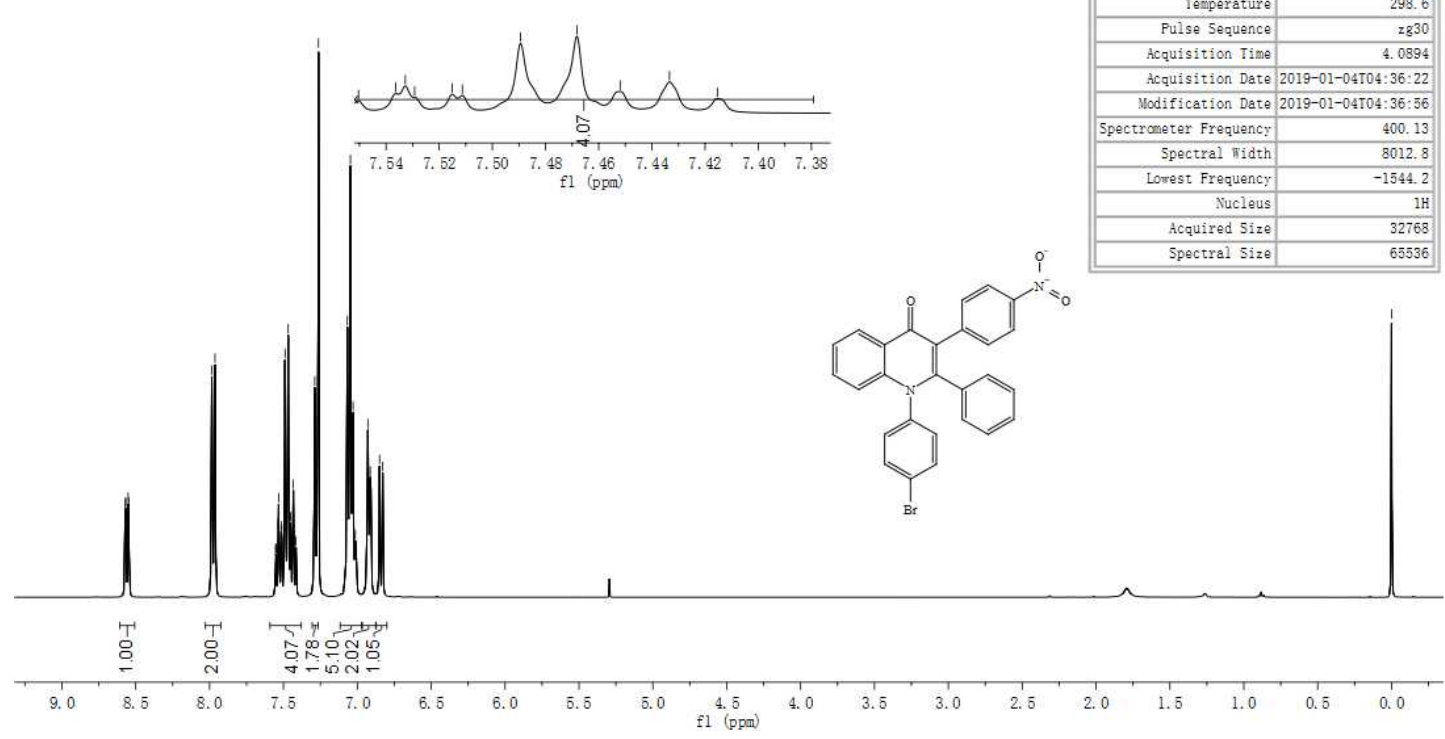

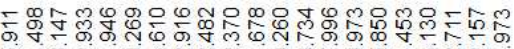

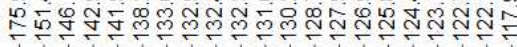

คำ

사요
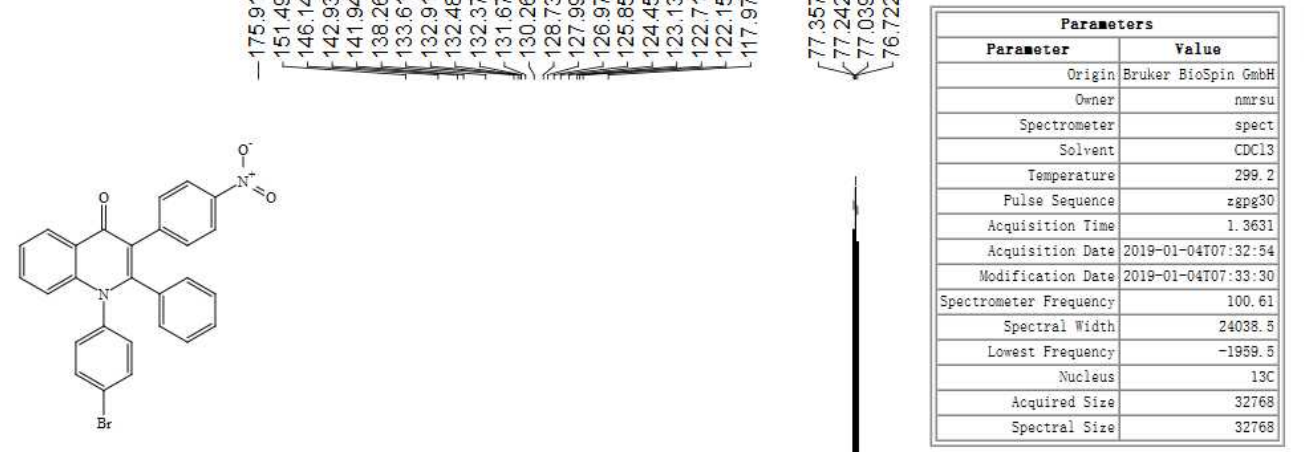

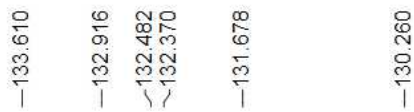
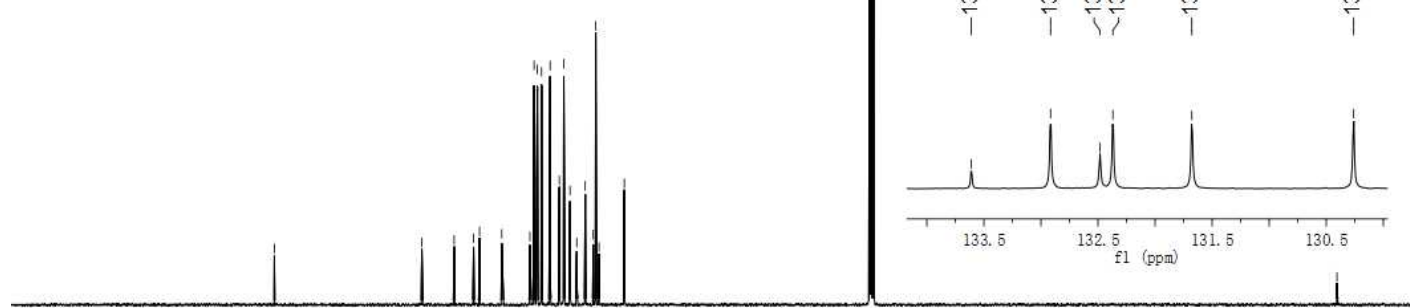

$\begin{array}{llllllllllll}210 & 200 & 190 & 180 & 170 & 160 & 150 & 140 & 130 & 120 & 110 & \underset{\mathrm{fl}}{100}(\mathrm{ppm})\end{array}$ 
1-(4-Chlorophenyl)-3-(4-nitrophenyl)-2-phenylquinolin-4(1H)-one (3e)

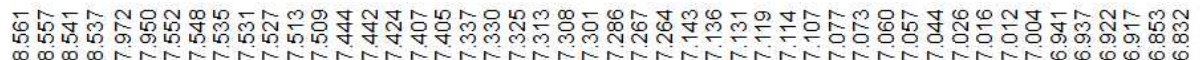

¿
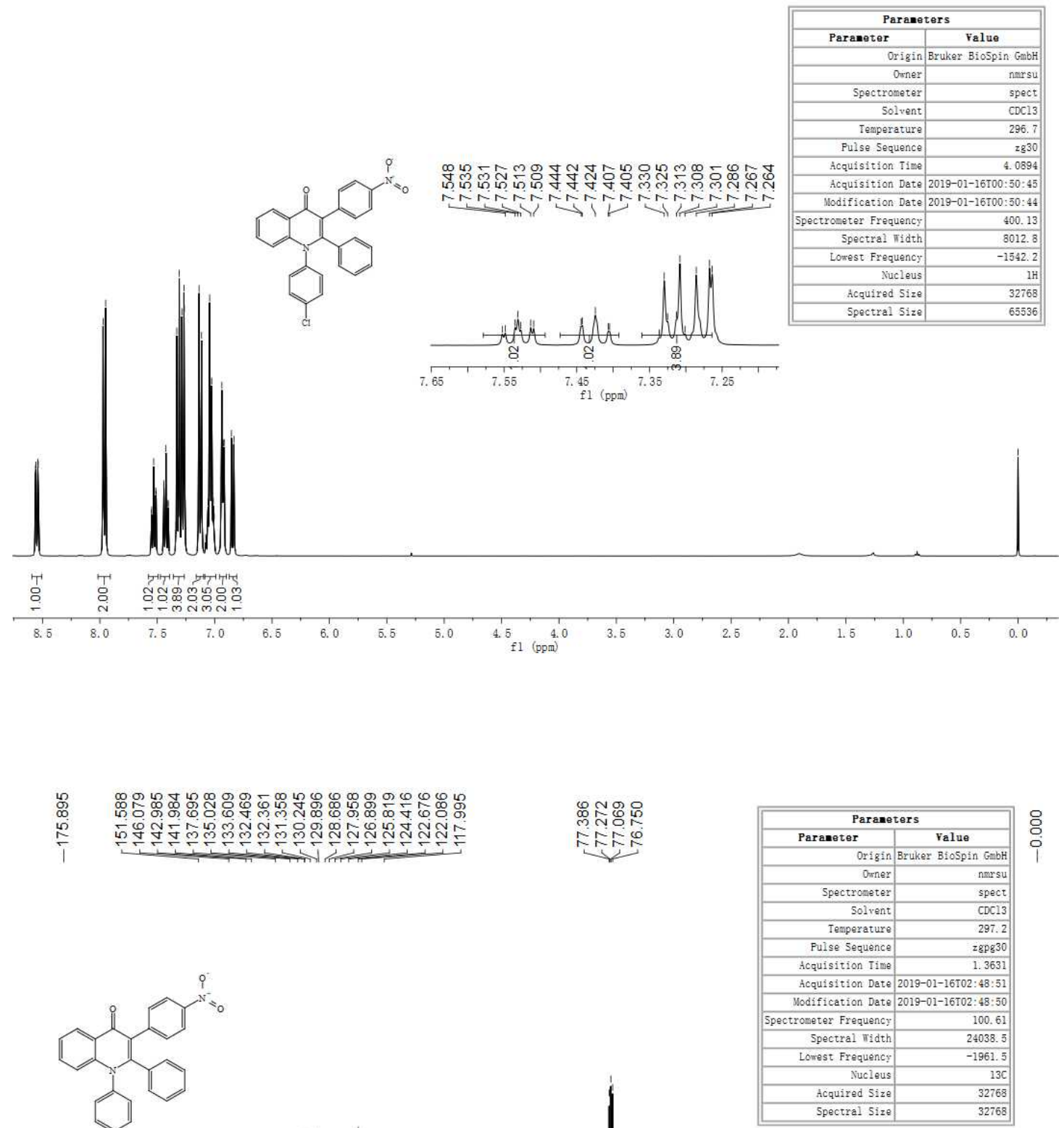

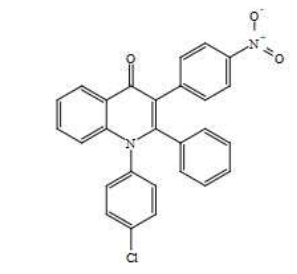

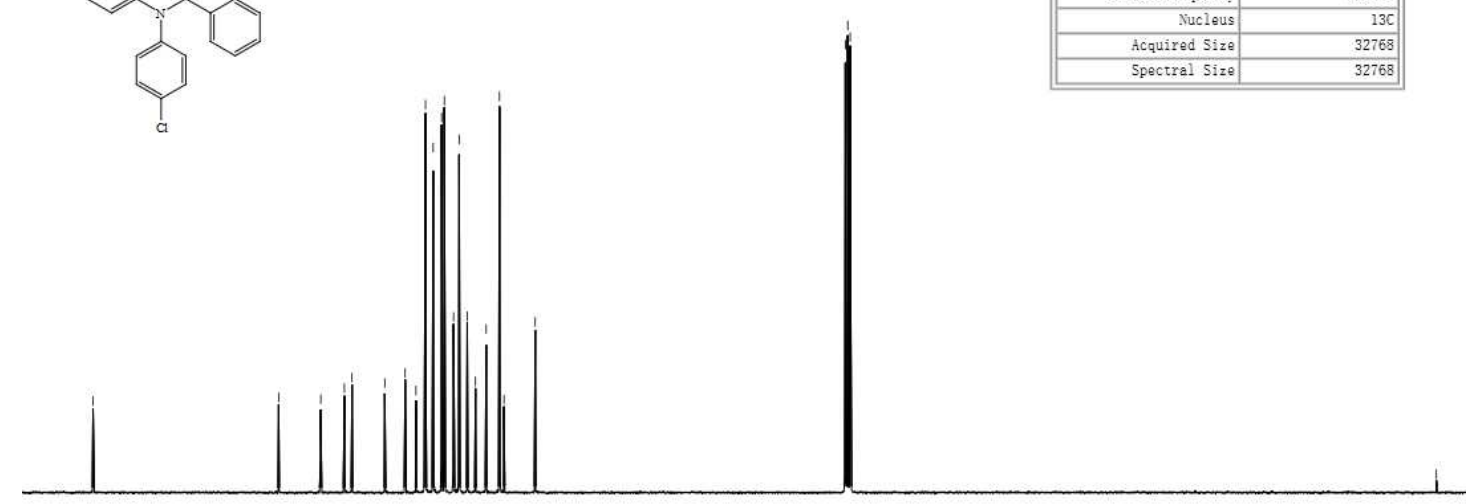

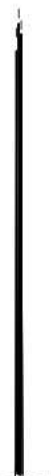

음

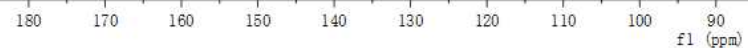


1-(4-Fluorophenyl)-3-(4-nitrophenyl)-2-phenylquinolin-4(1H)-one (3f)
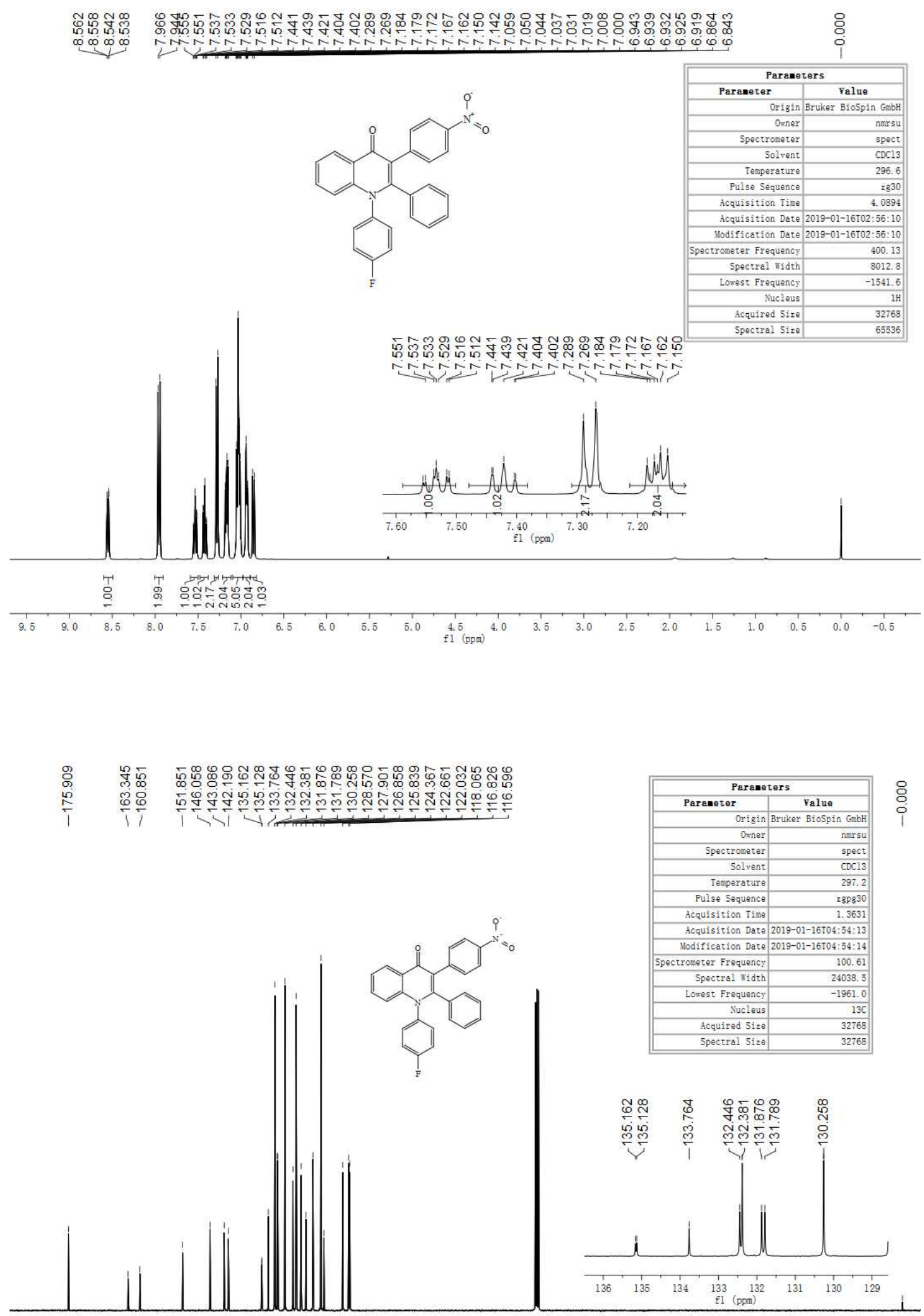


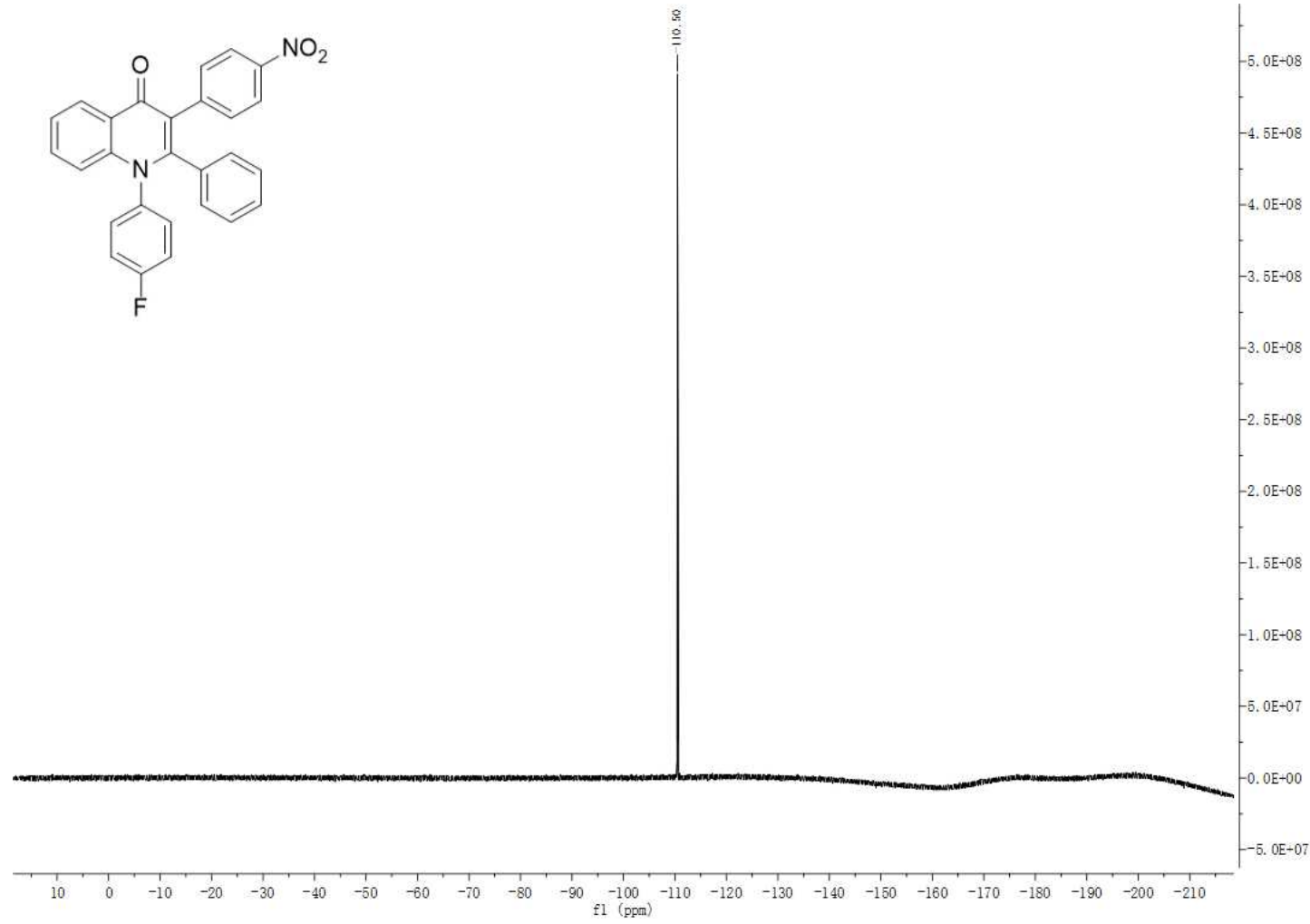

1-(4-Iodophenyl)-3-(4-nitrophenyl)-2-phenylquinolin-4(1H)-one (3g)

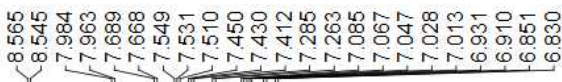

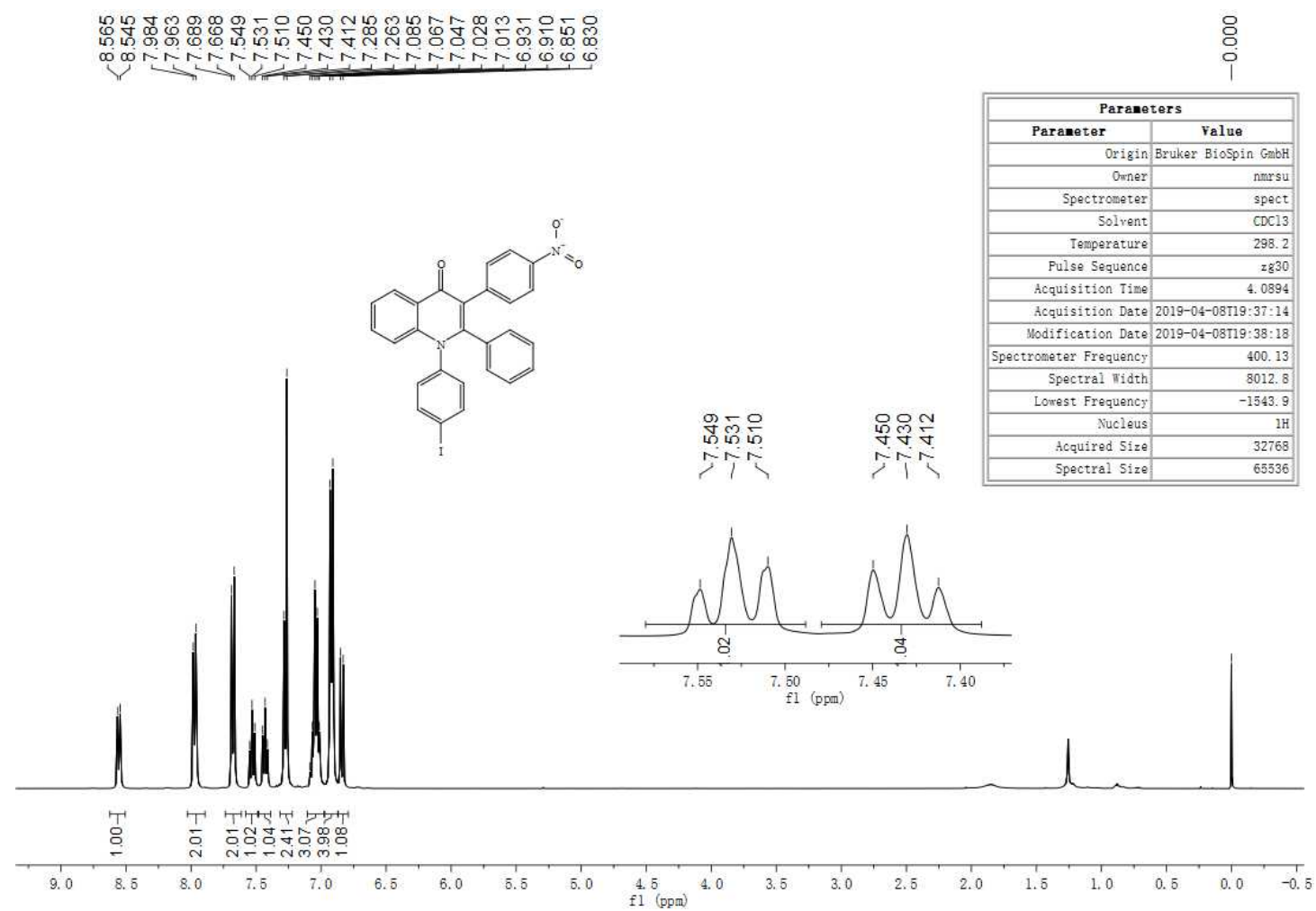



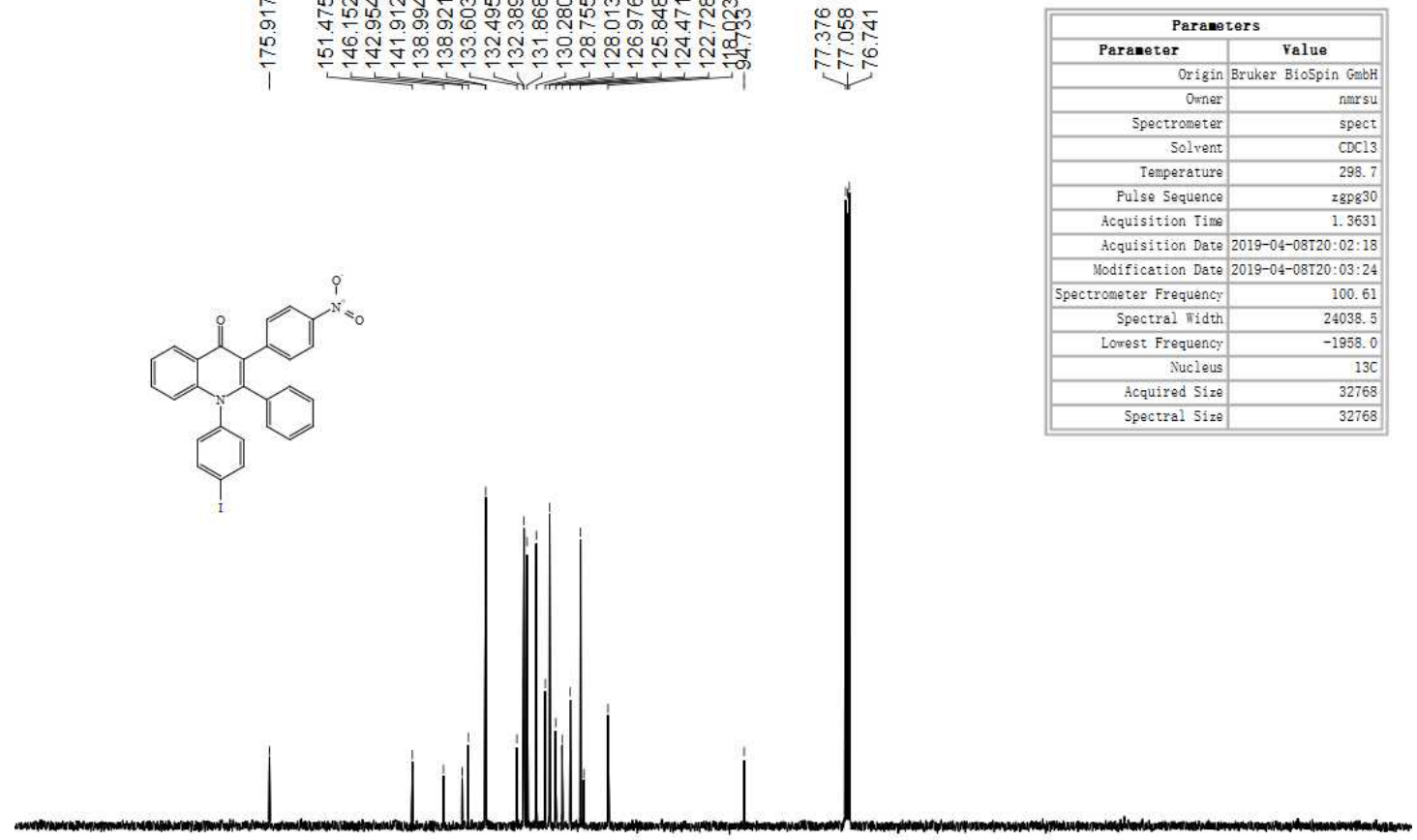

$210 \quad 200$

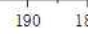

$80 \quad 170 \quad 160 \quad 150$

1-Benzyl-3-(4-nitrophenyl)-2-phenylquinolin-4(1H)-one (3h)

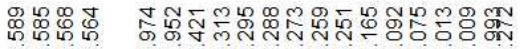

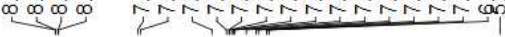
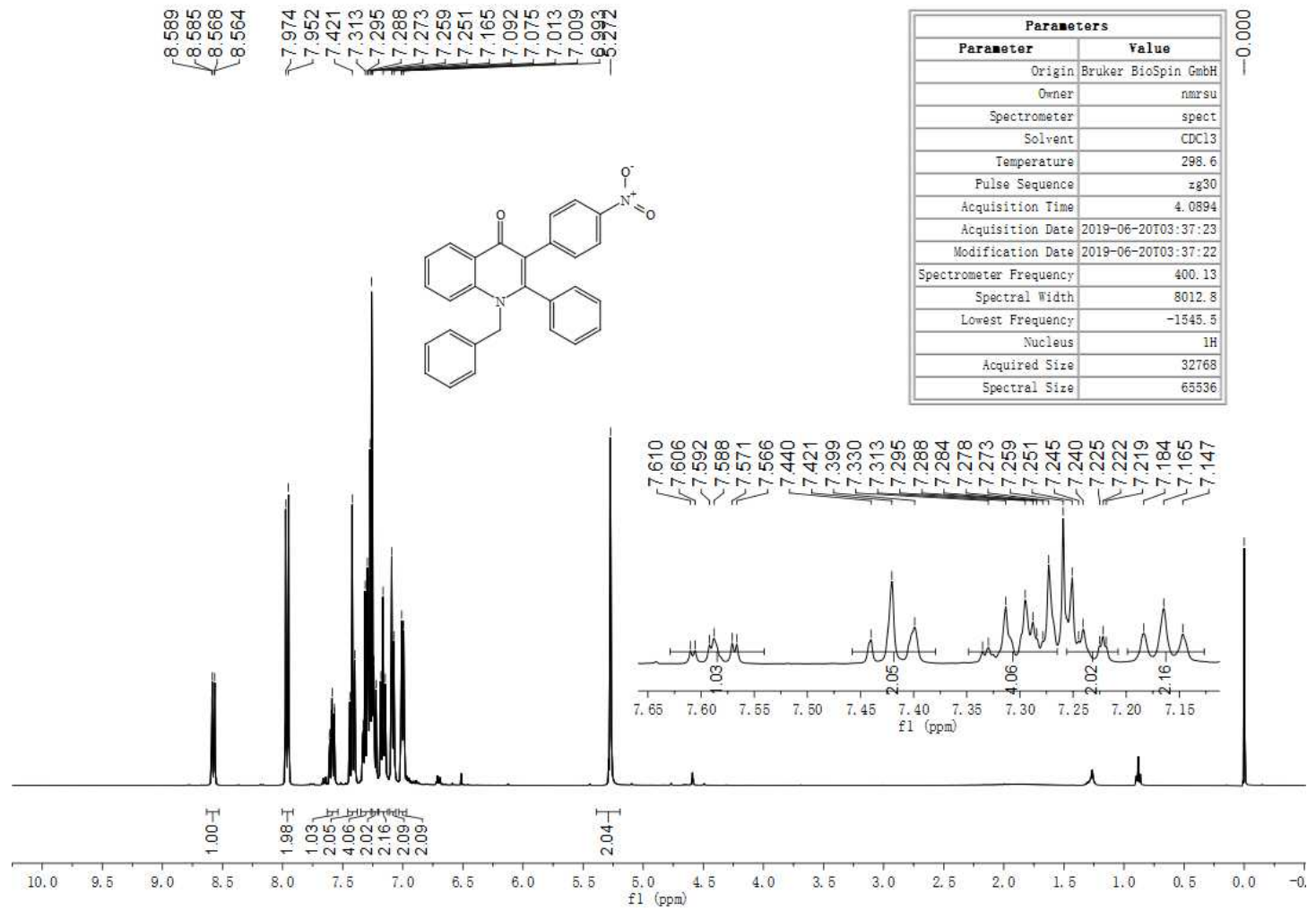


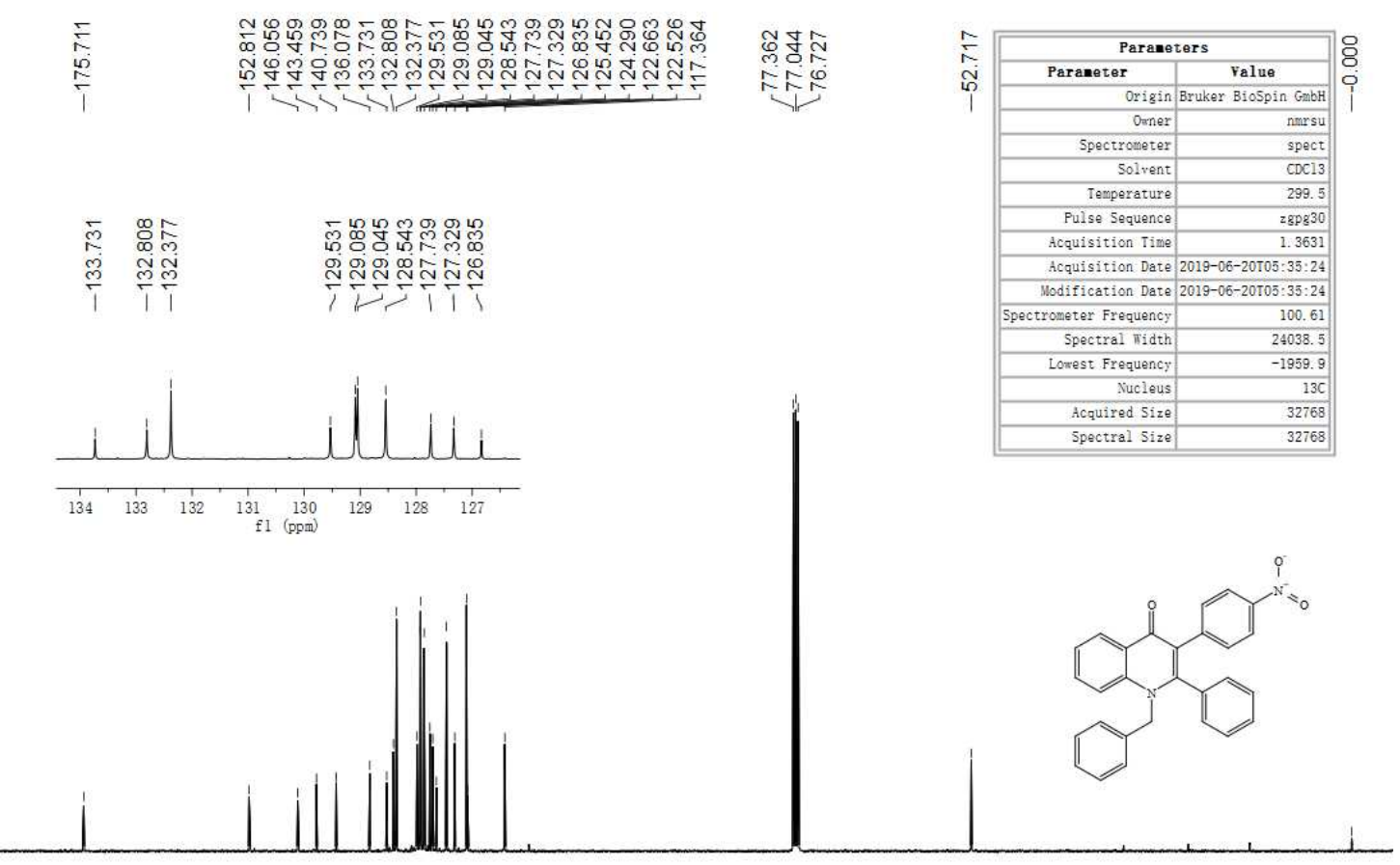

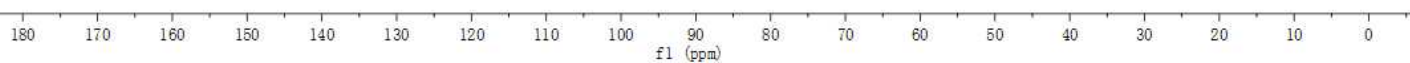

1-(Naphthalen-2-yl)-3-(4-nitrophenyl)-2-phenylquinolin-4(1H)-one (3i)

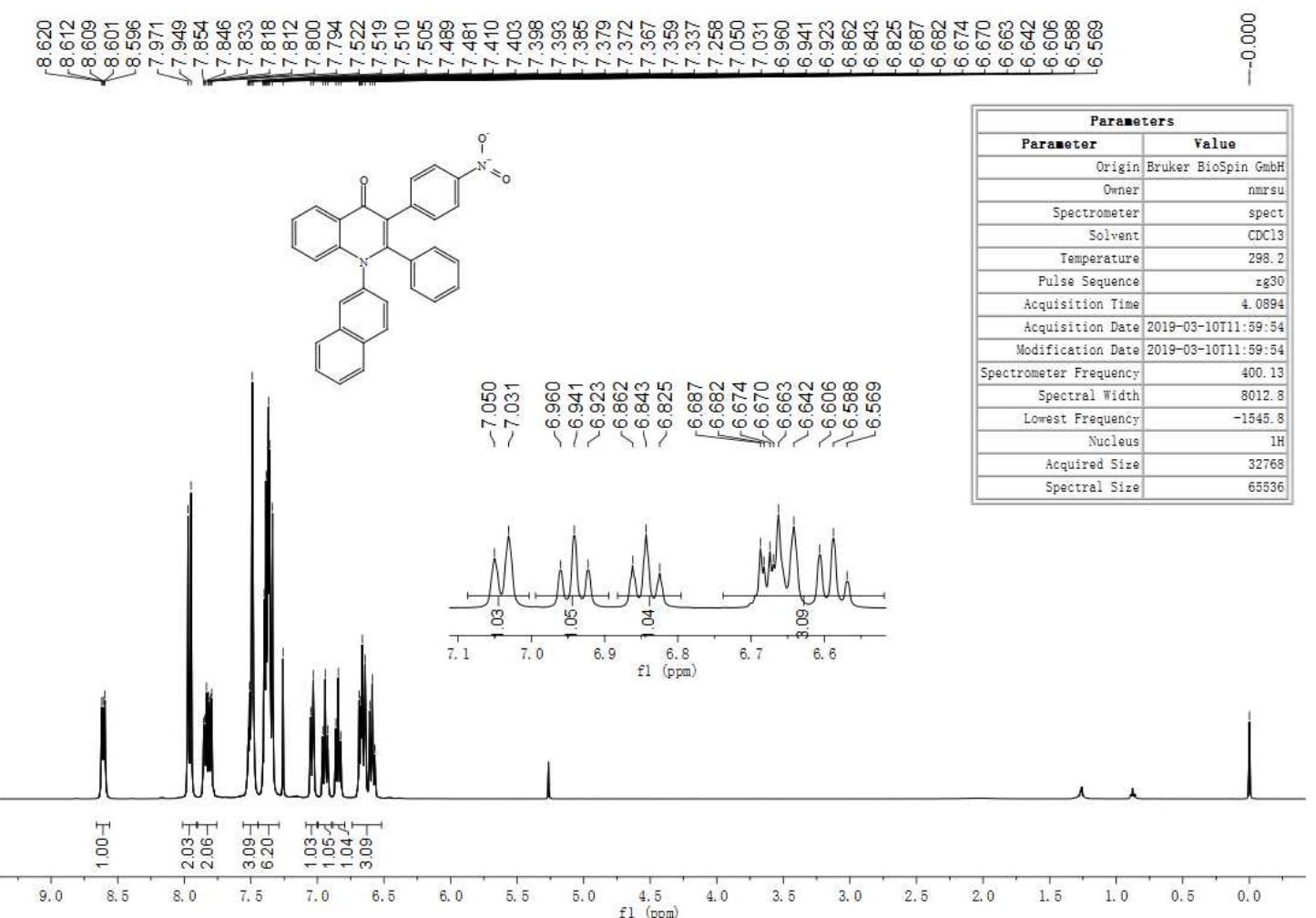



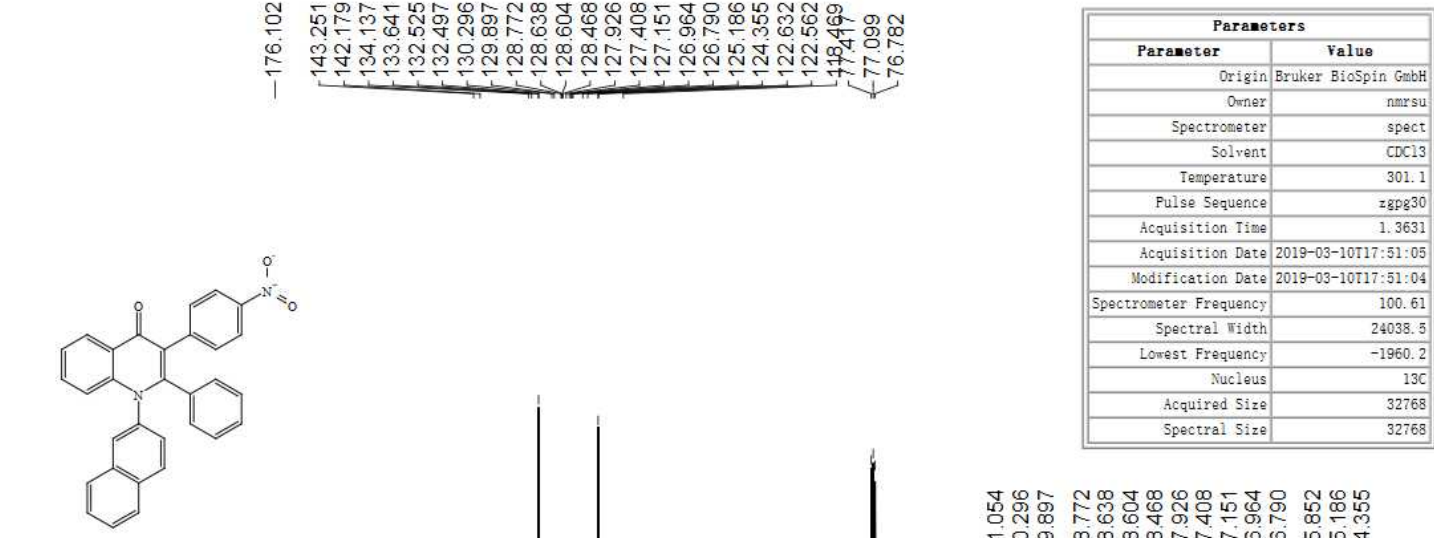

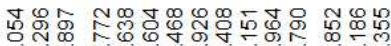
mర్లి

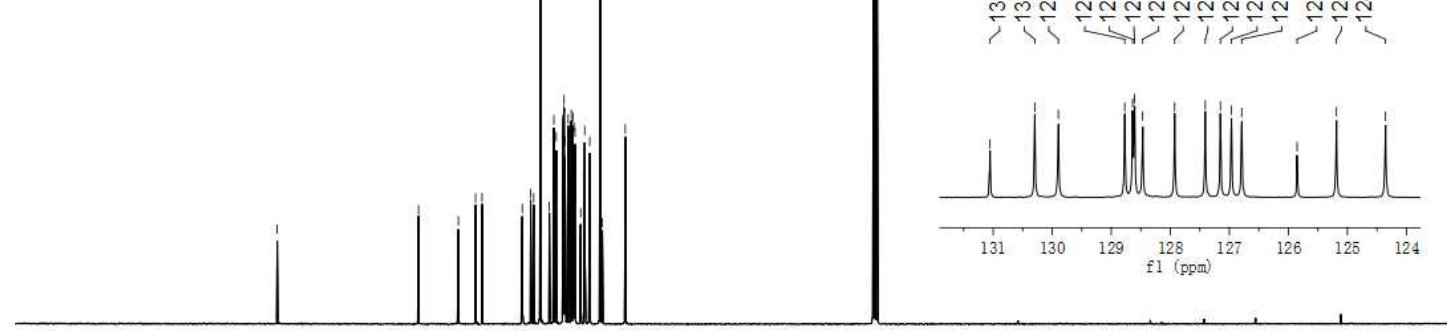

$\begin{array}{lllllll}210 & 200 & 190 & 180 & 170 & 160 & 150\end{array}$

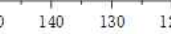

$10 \stackrel{100}{\mathrm{fl}}(\mathrm{ppm})$

1-Methyl-3-(4-nitrophenyl)-2-phenylquinolin-4(1H)-one (3j)

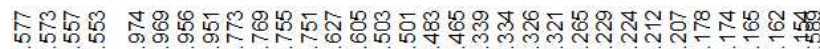
$\underbrace{\infty} \infty$
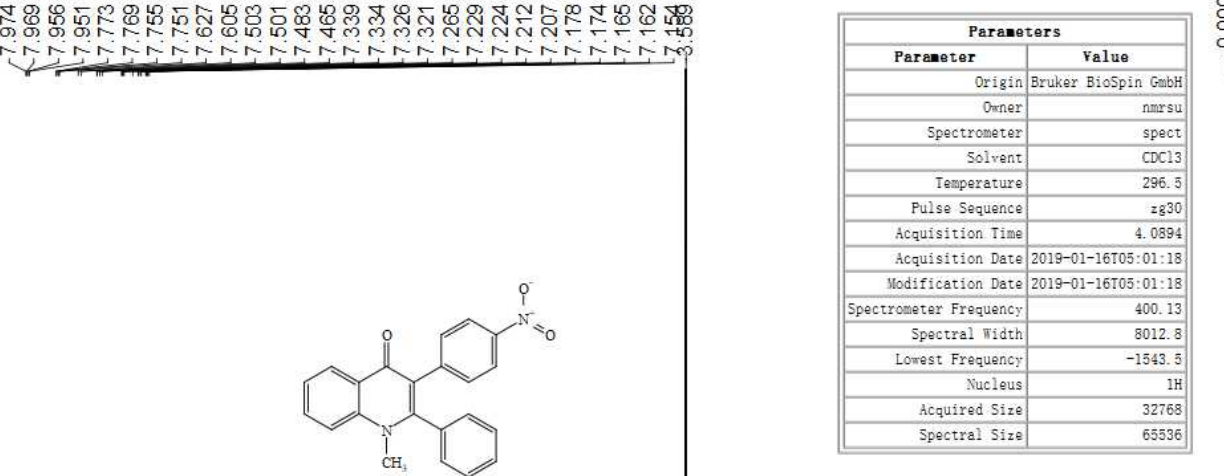

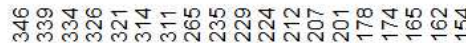
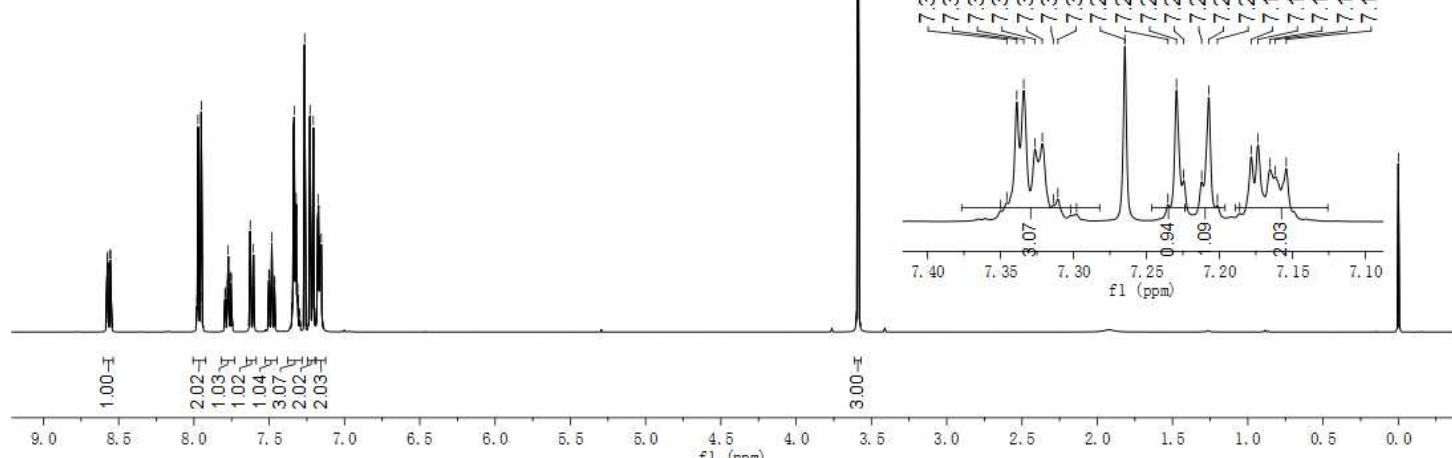


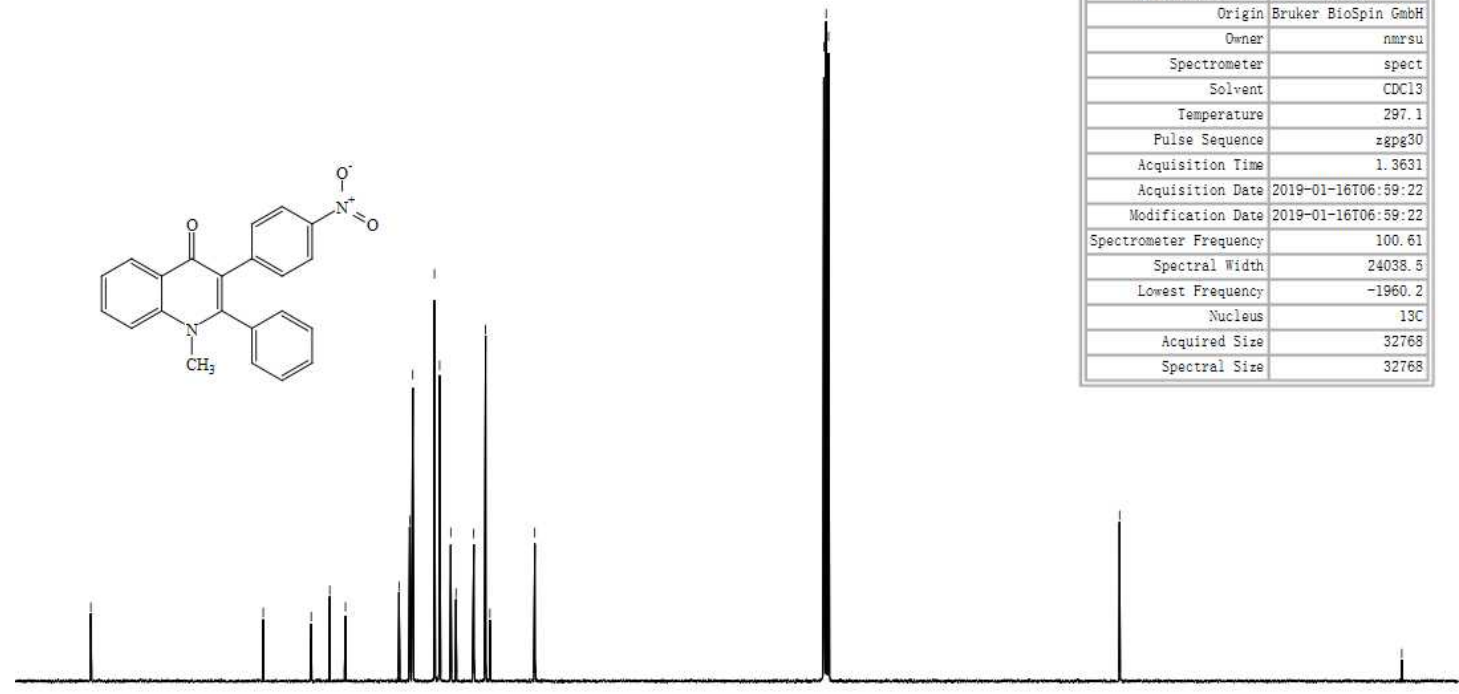

1-Ethyl-3-(4-nitrophenyl)-2-phenylquinolin-4(1H)-one (3k)

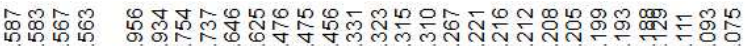

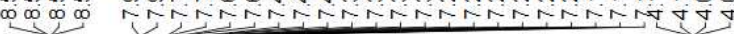
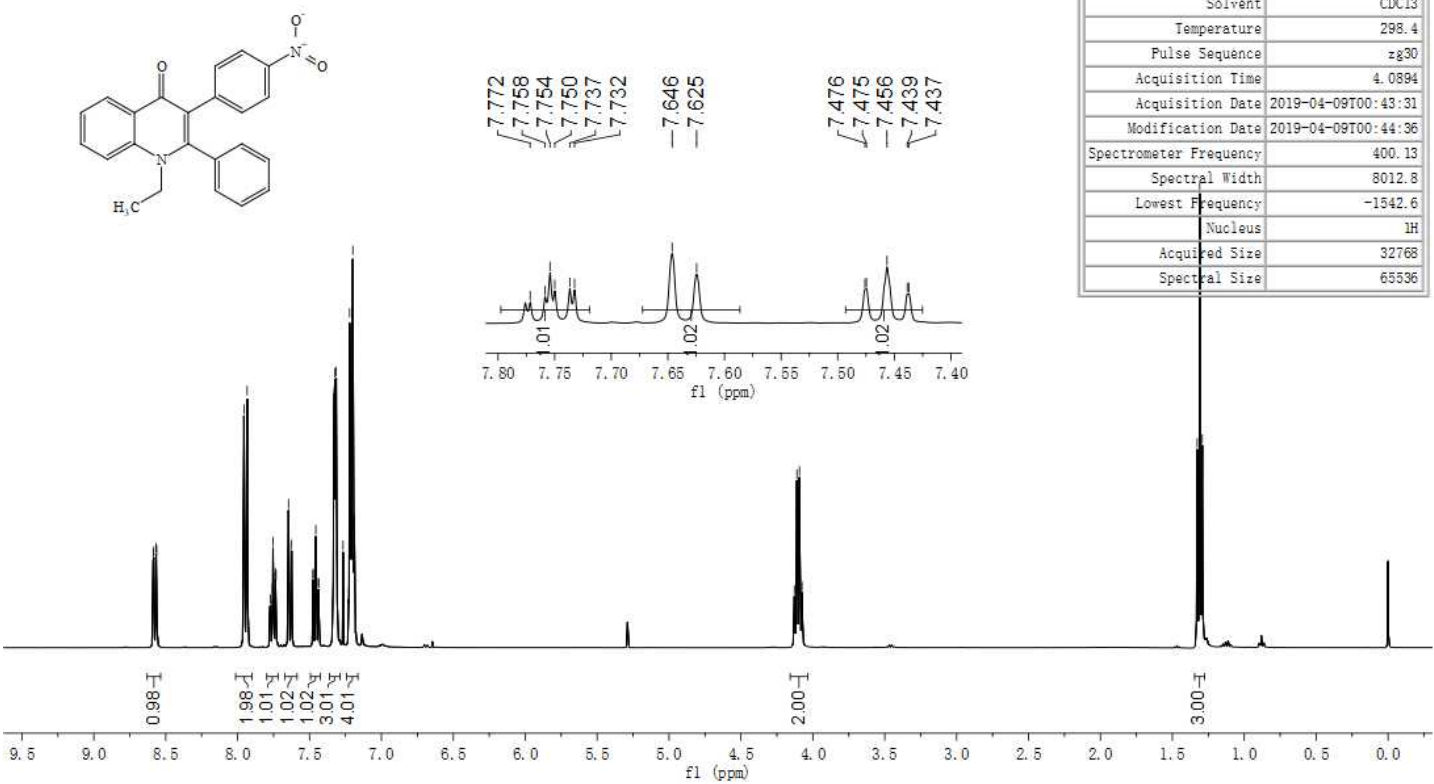

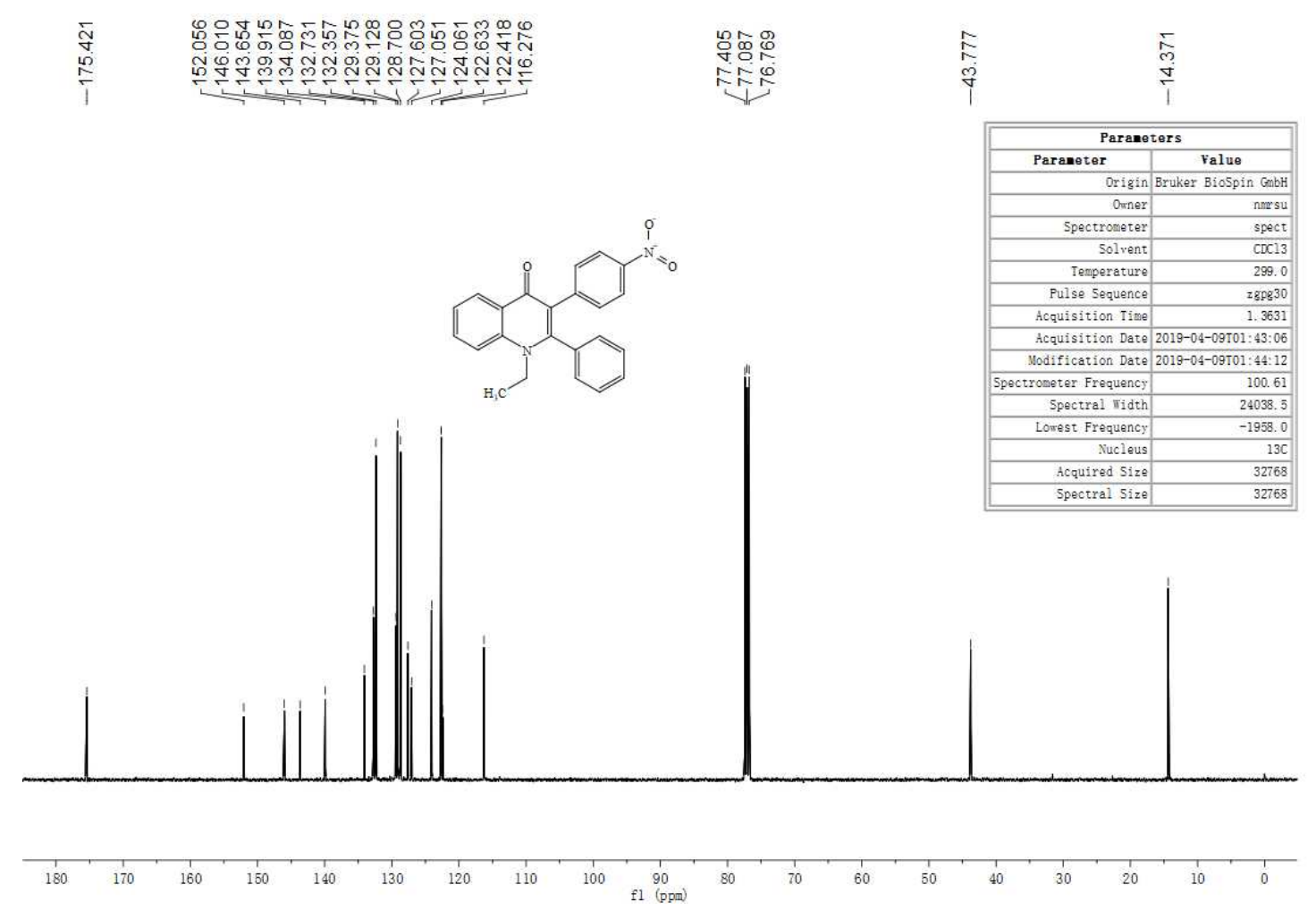

\section{3-(4-Nitrophenyl)-2-phenyl-1-propylquinolin-4(1H)-one (3l)}

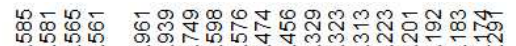

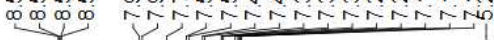
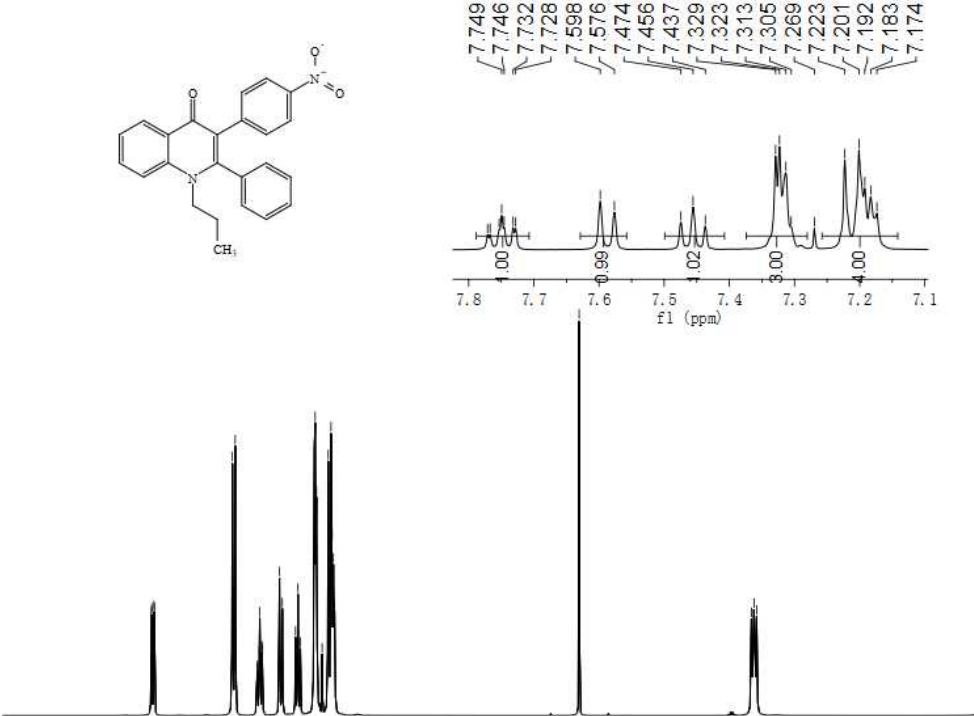

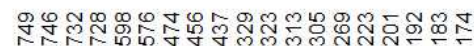

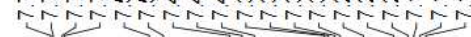

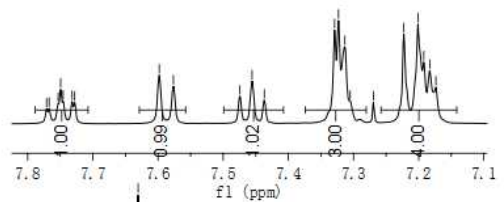

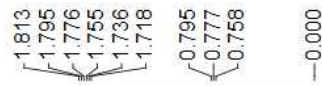
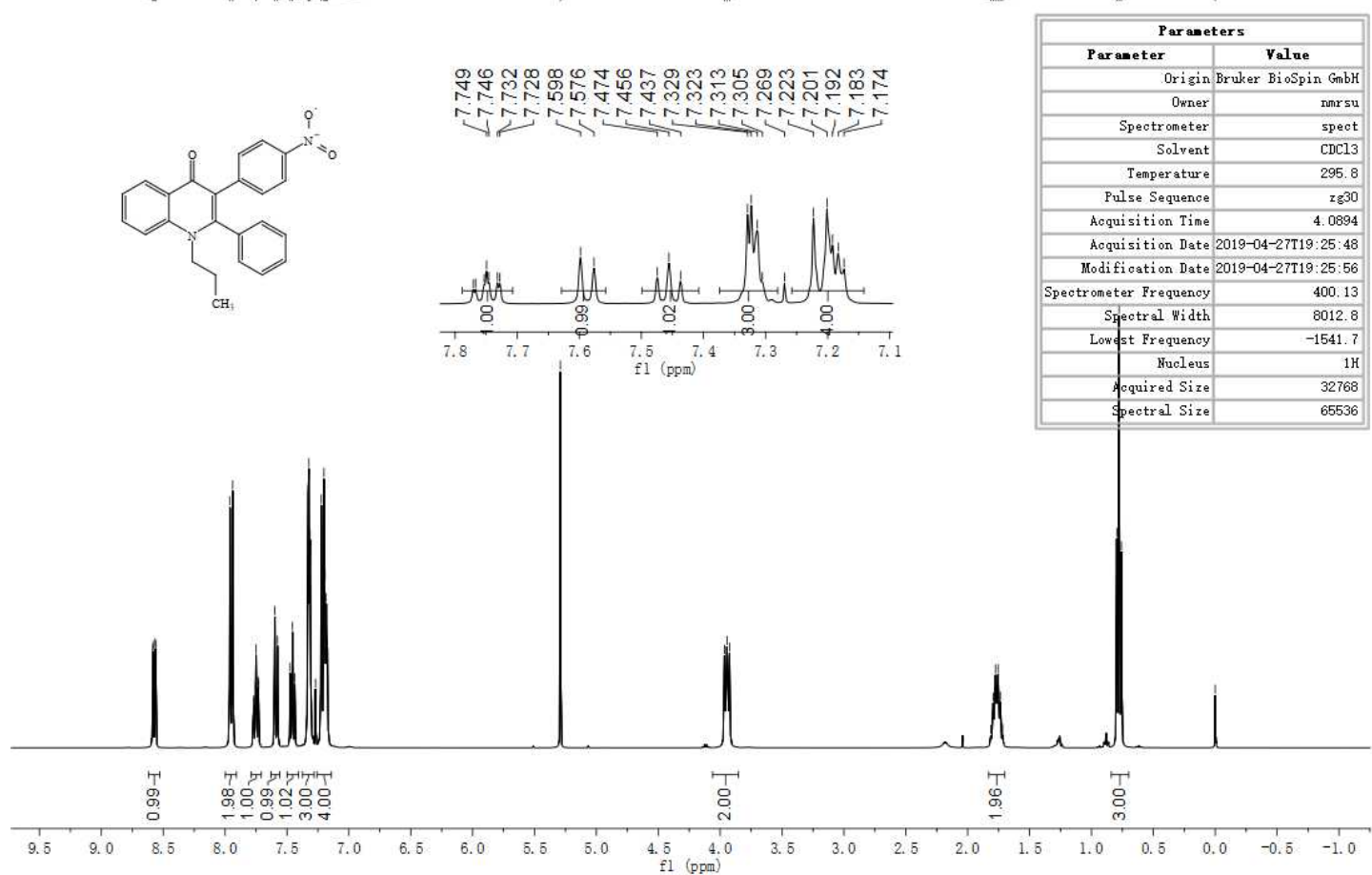


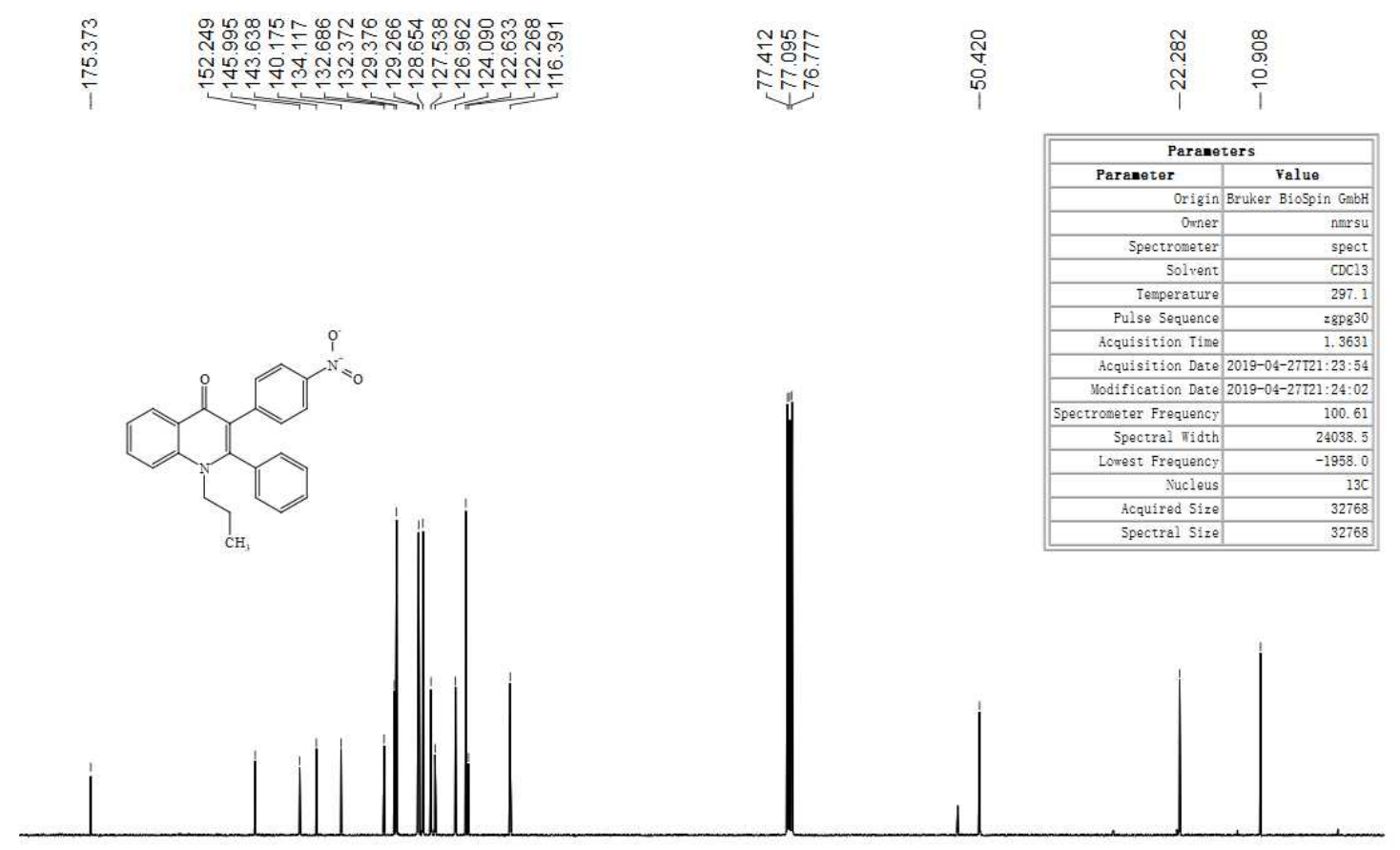

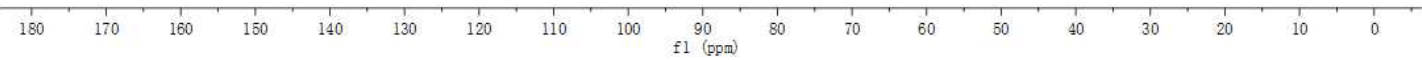

\section{1,2-Diphenyl-3-(4-(trifluoromethyl)phenyl)quinolin-4(1H)-one (3m)}

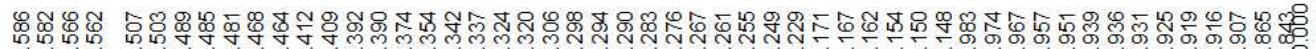

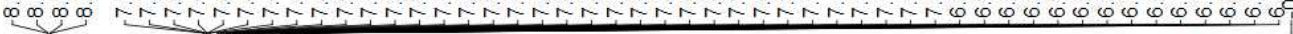
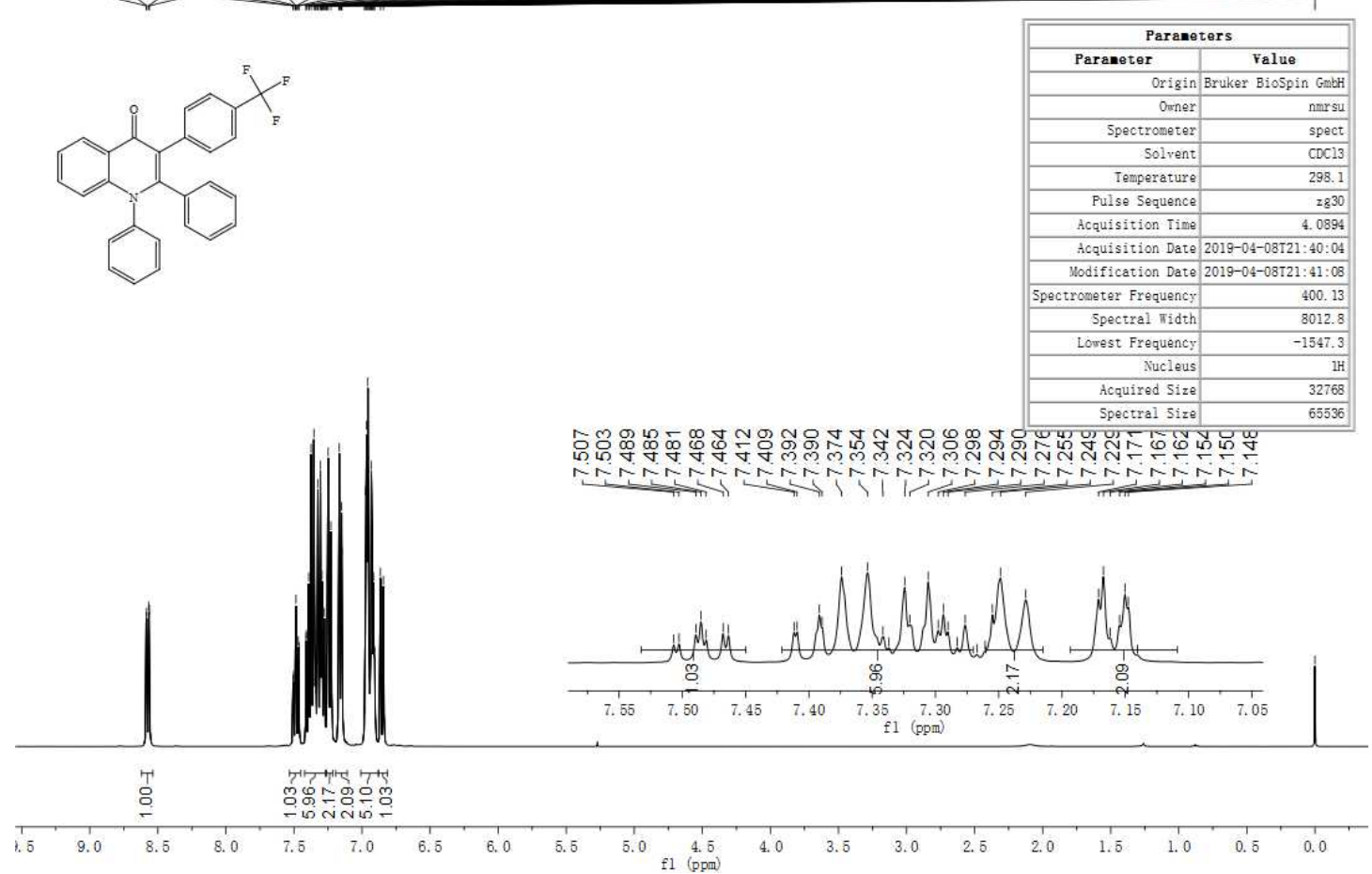

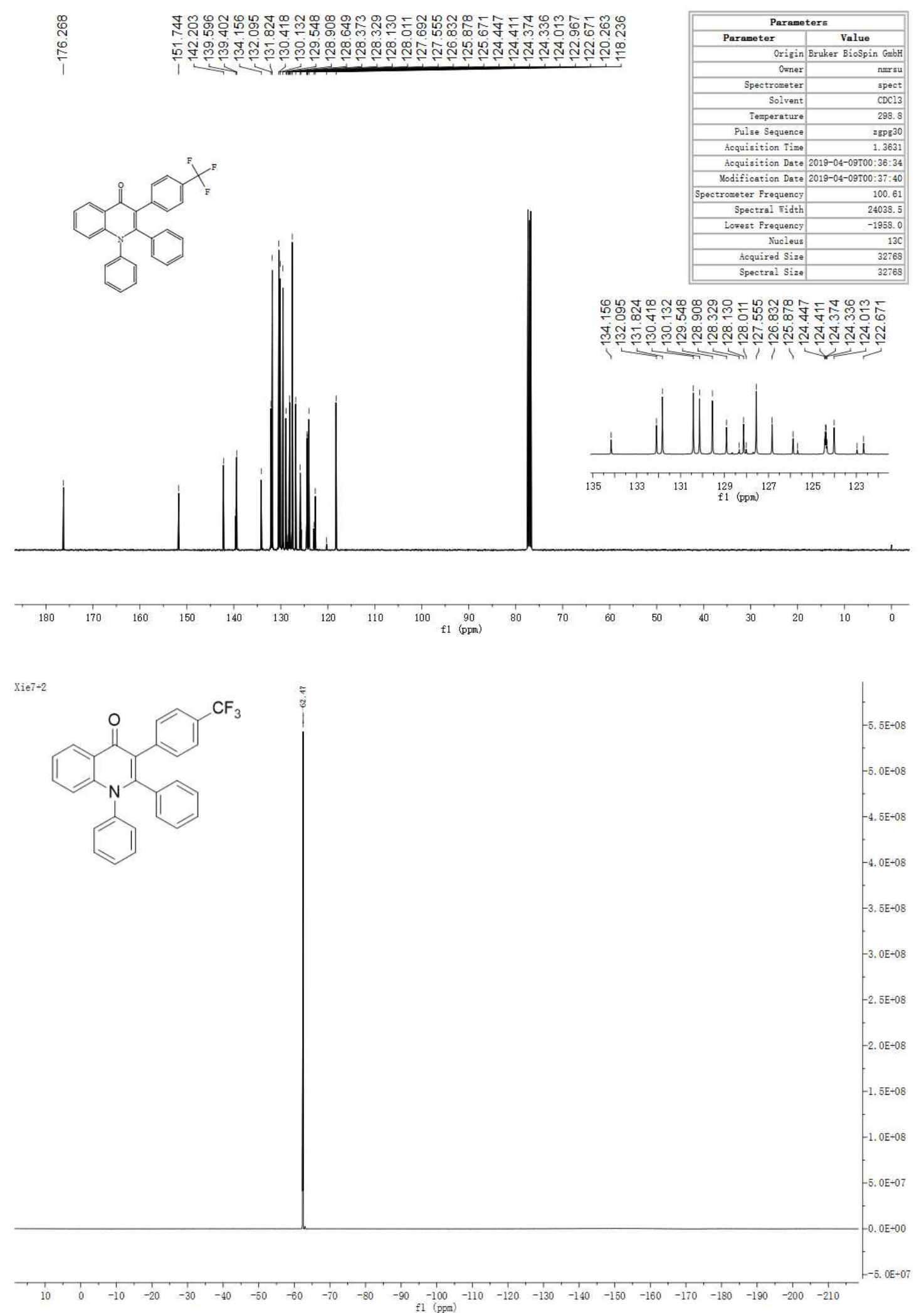
4-(4-Oxo-1,2-diphenyl-1,4-dihydroquinolin-3-yl)benzonitrile (3n)

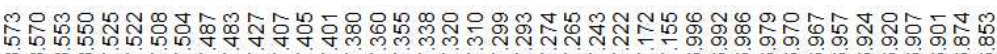

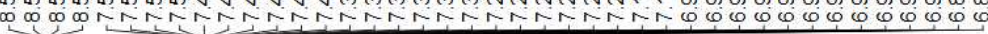

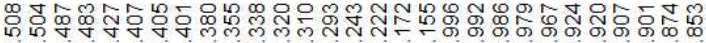

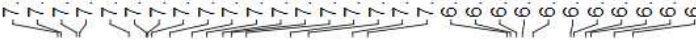
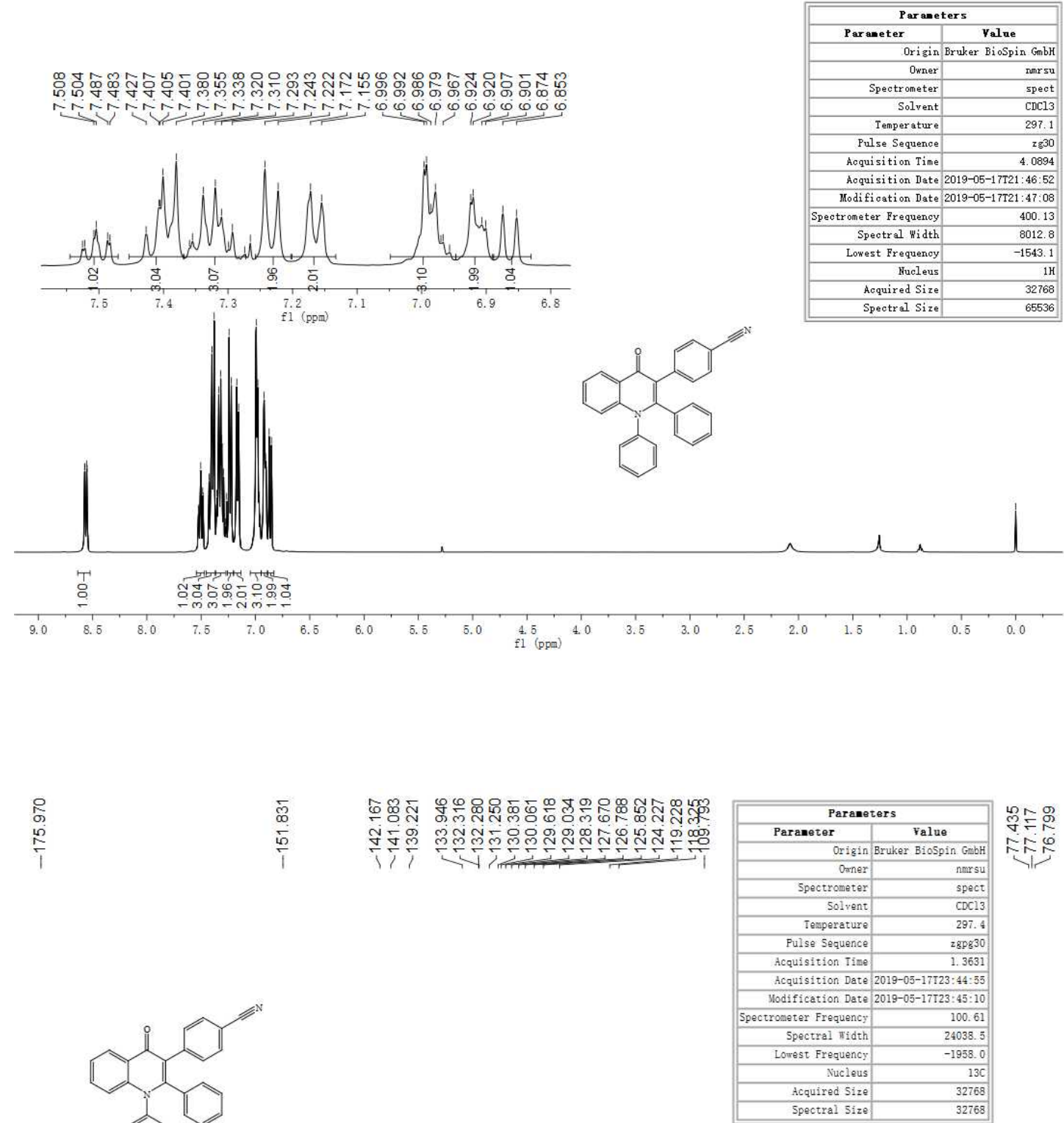

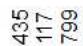

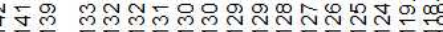<smiles>CC1CCCCC1C1CCC(C)C(c2ccccc2)C1C1CCCCC1</smiles>

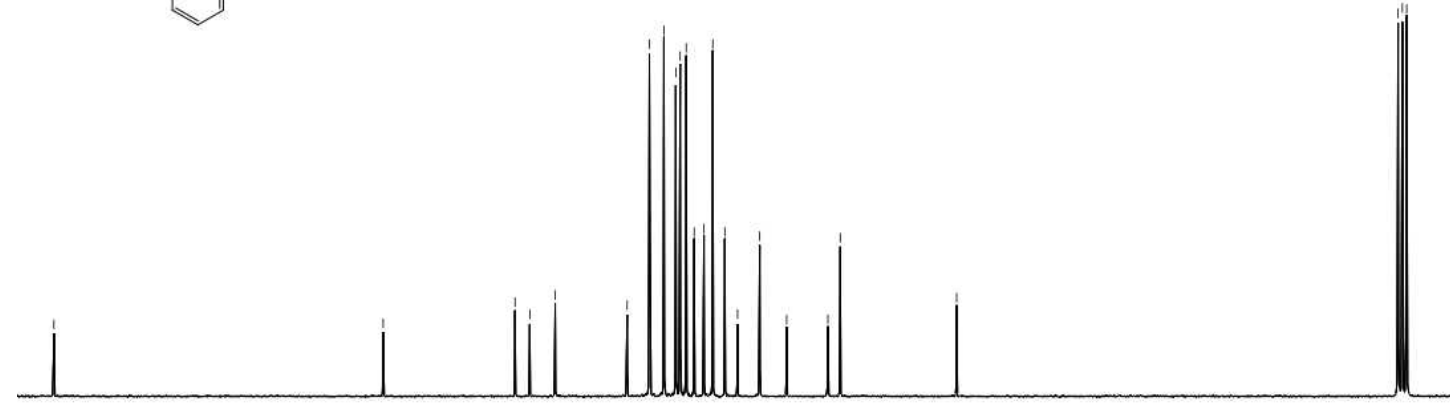

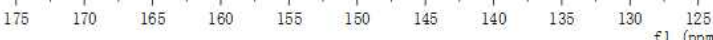
$120 \quad 115 \quad 110$ 75 
3-(2-Nitrophenyl)-1,2-diphenylquinolin-4(1H)-one (3o)

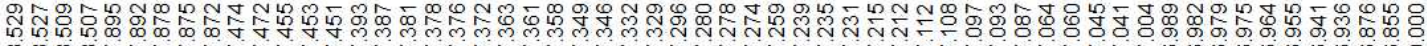

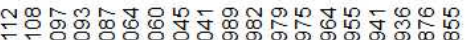

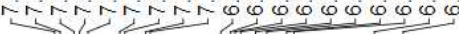
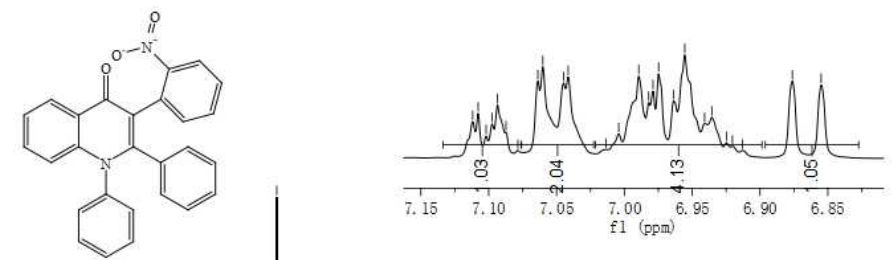

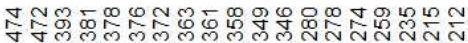

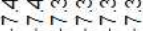
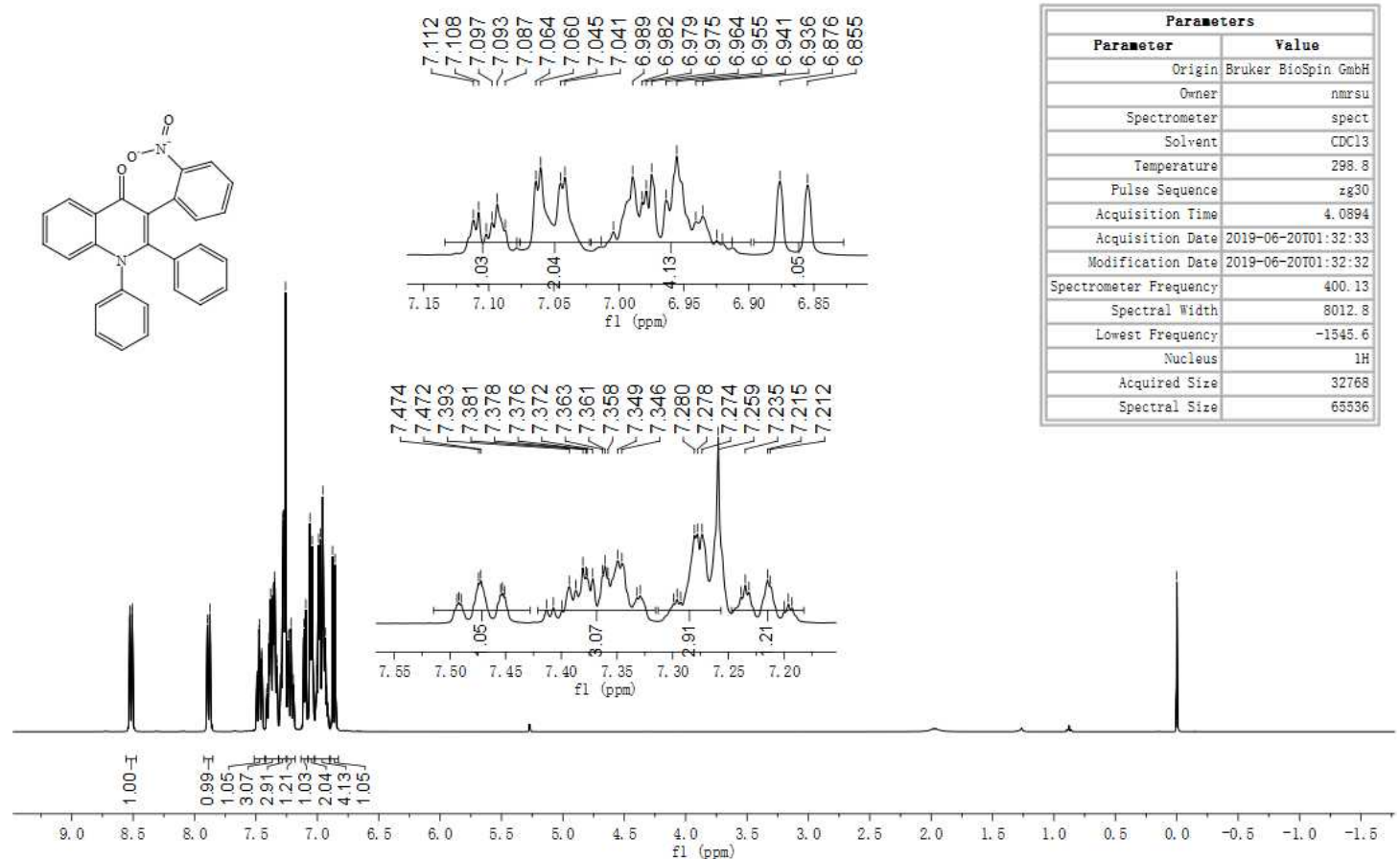

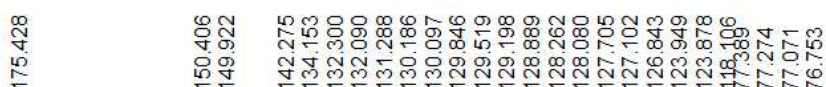

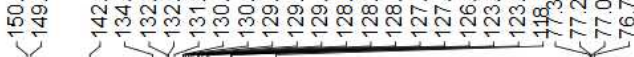
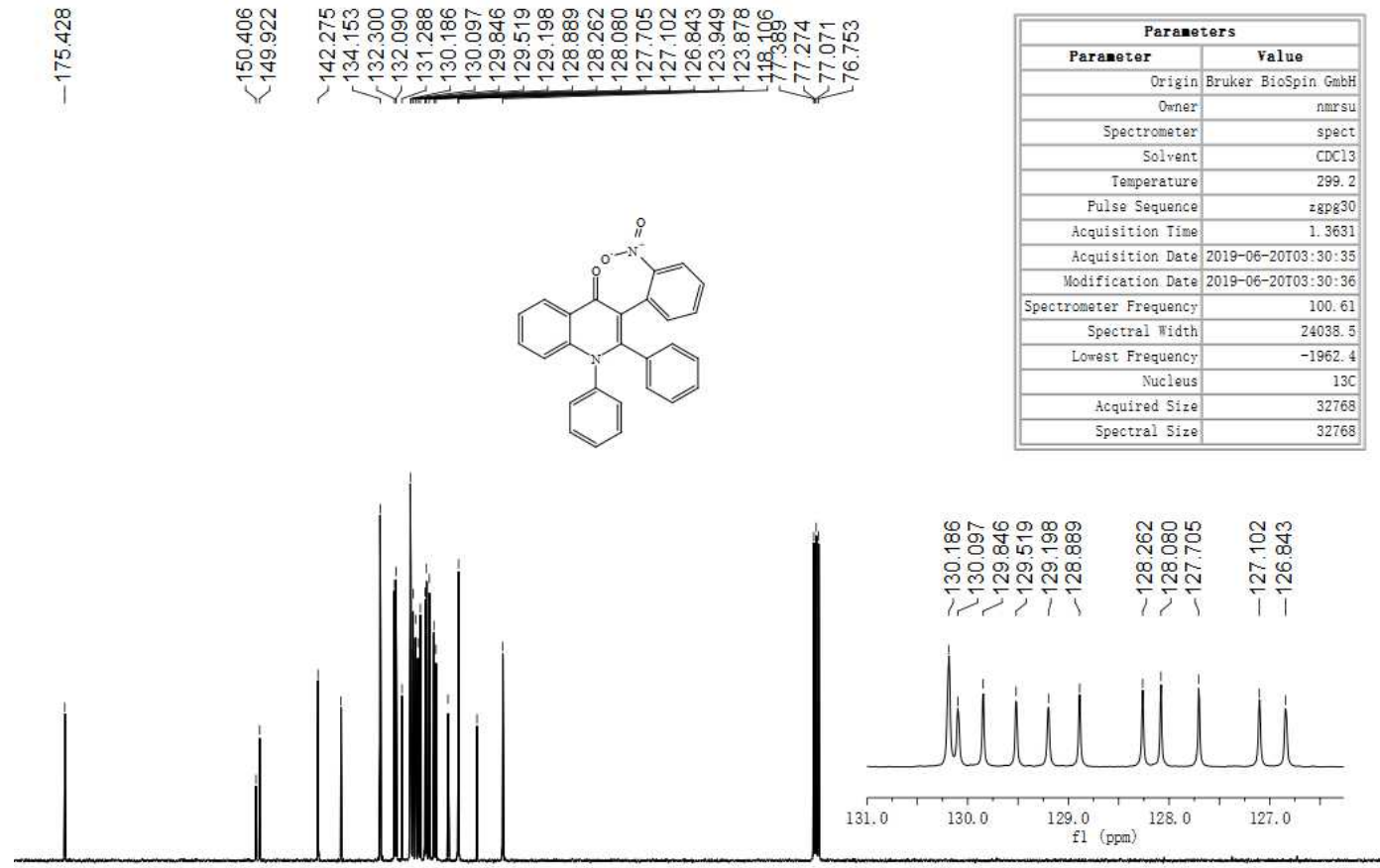

180

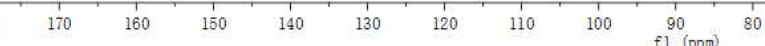


7-Methyl-3-(4-nitrophenyl)-1,2-diphenylquinolin-4(1H)-one (3p)

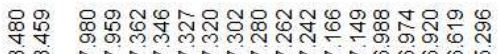

o
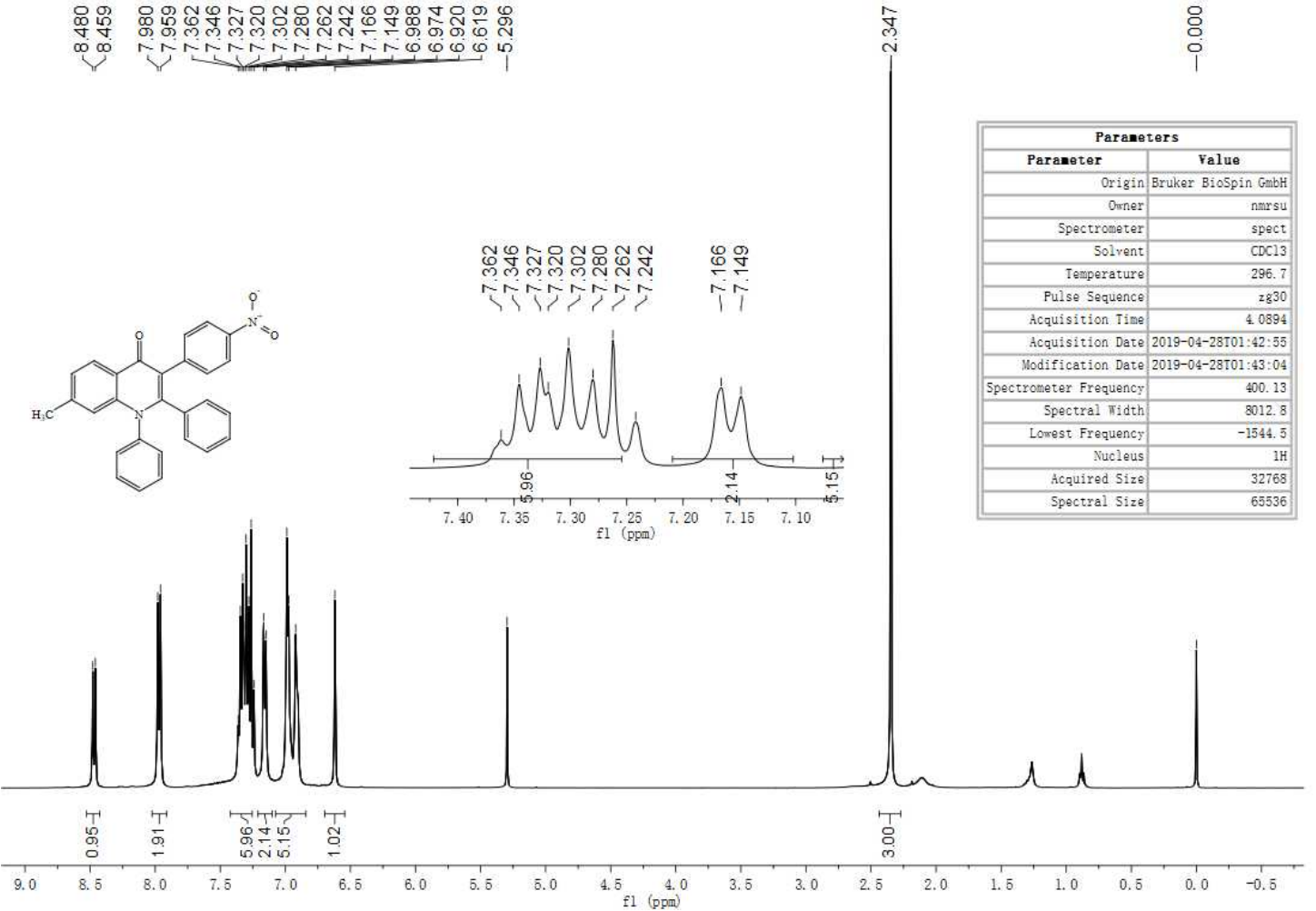

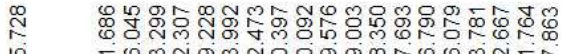

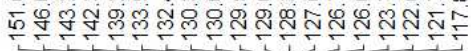
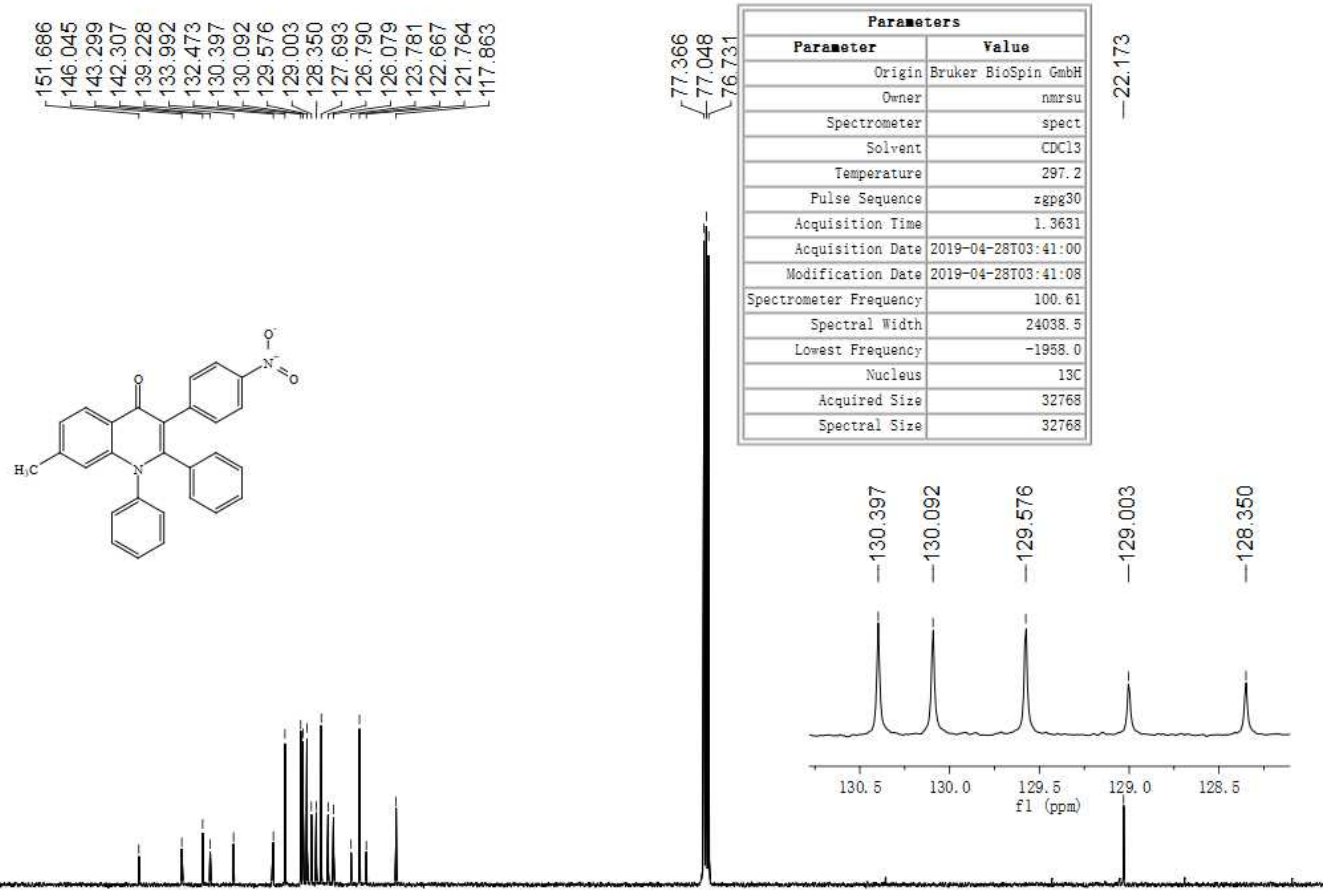
7-Methoxy-3-(4-nitrophenyl)-1,2-diphenylquinolin-4(1H)-one (3q)

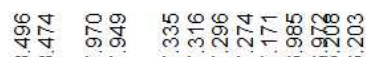

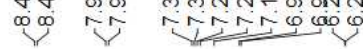

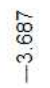

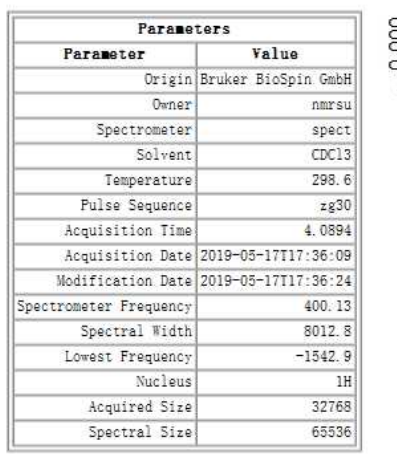

(1)

品
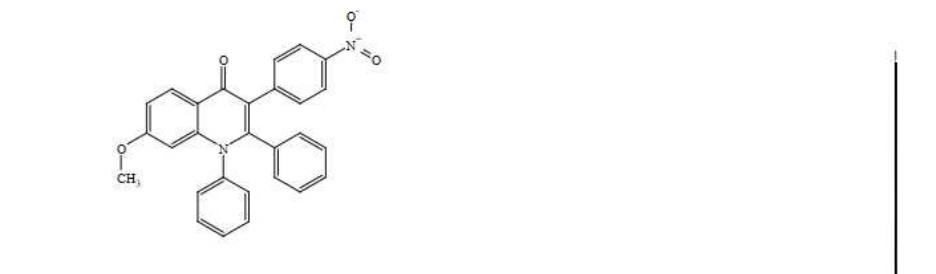

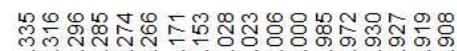

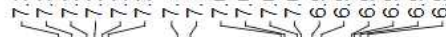
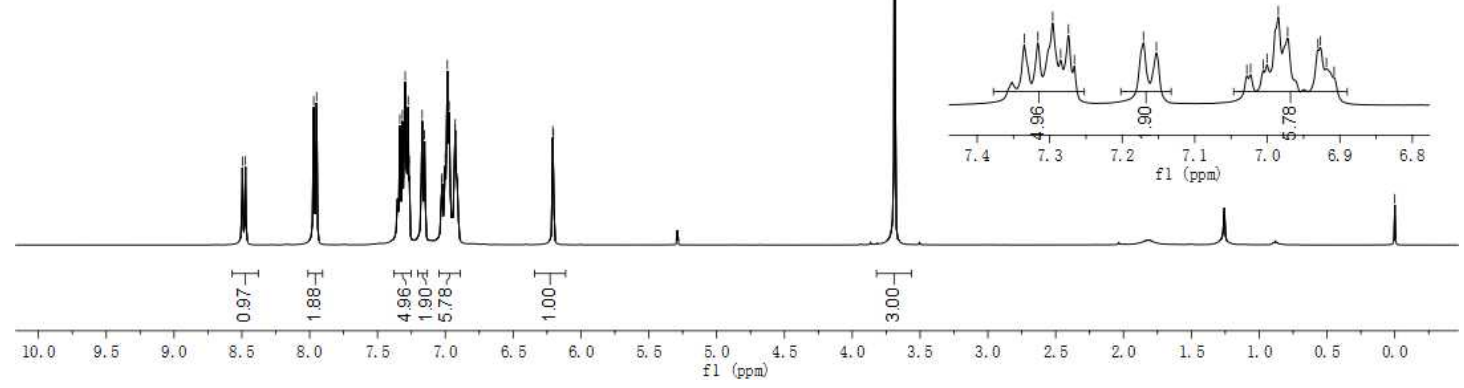

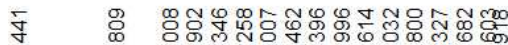

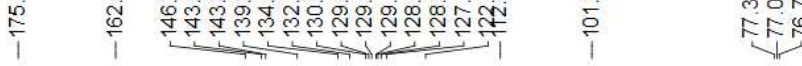
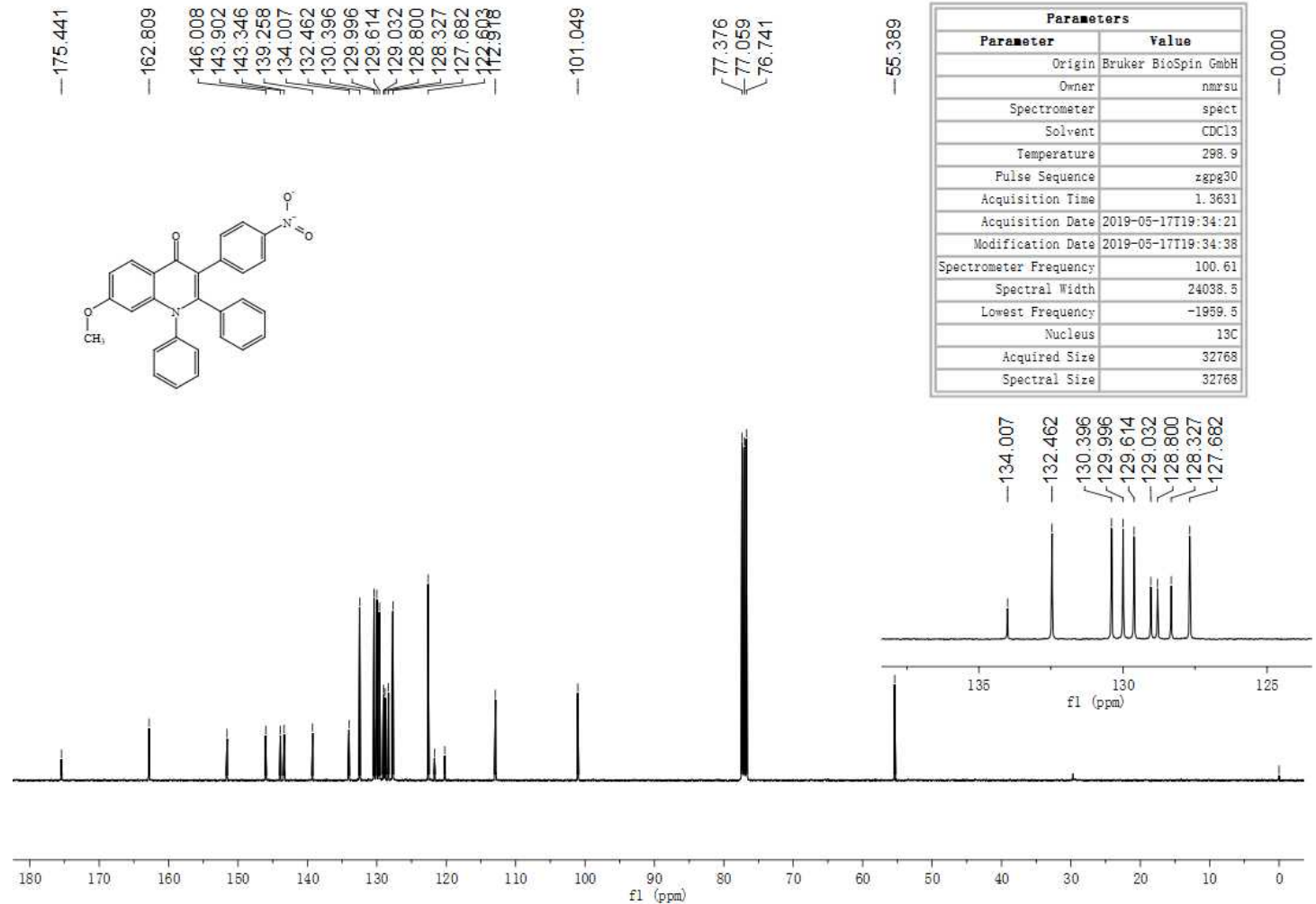
7-Chloro-3-(4-nitrophenyl)-1,2-diphenylquinolin-4(1H)-one (3r)

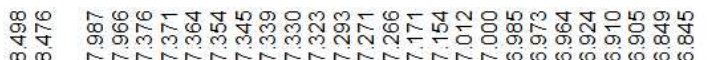
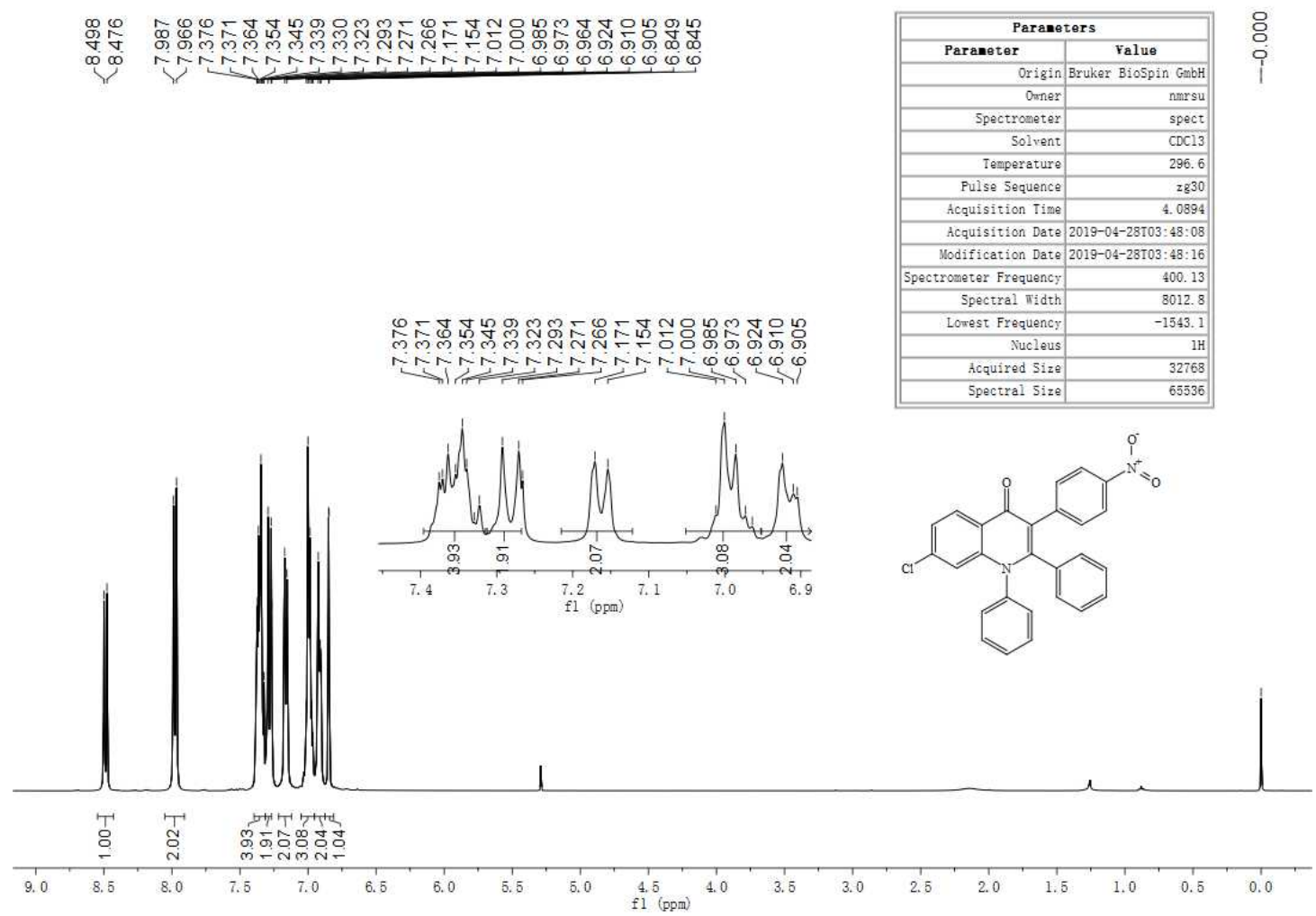

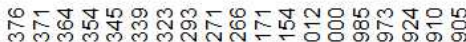

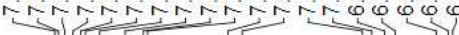
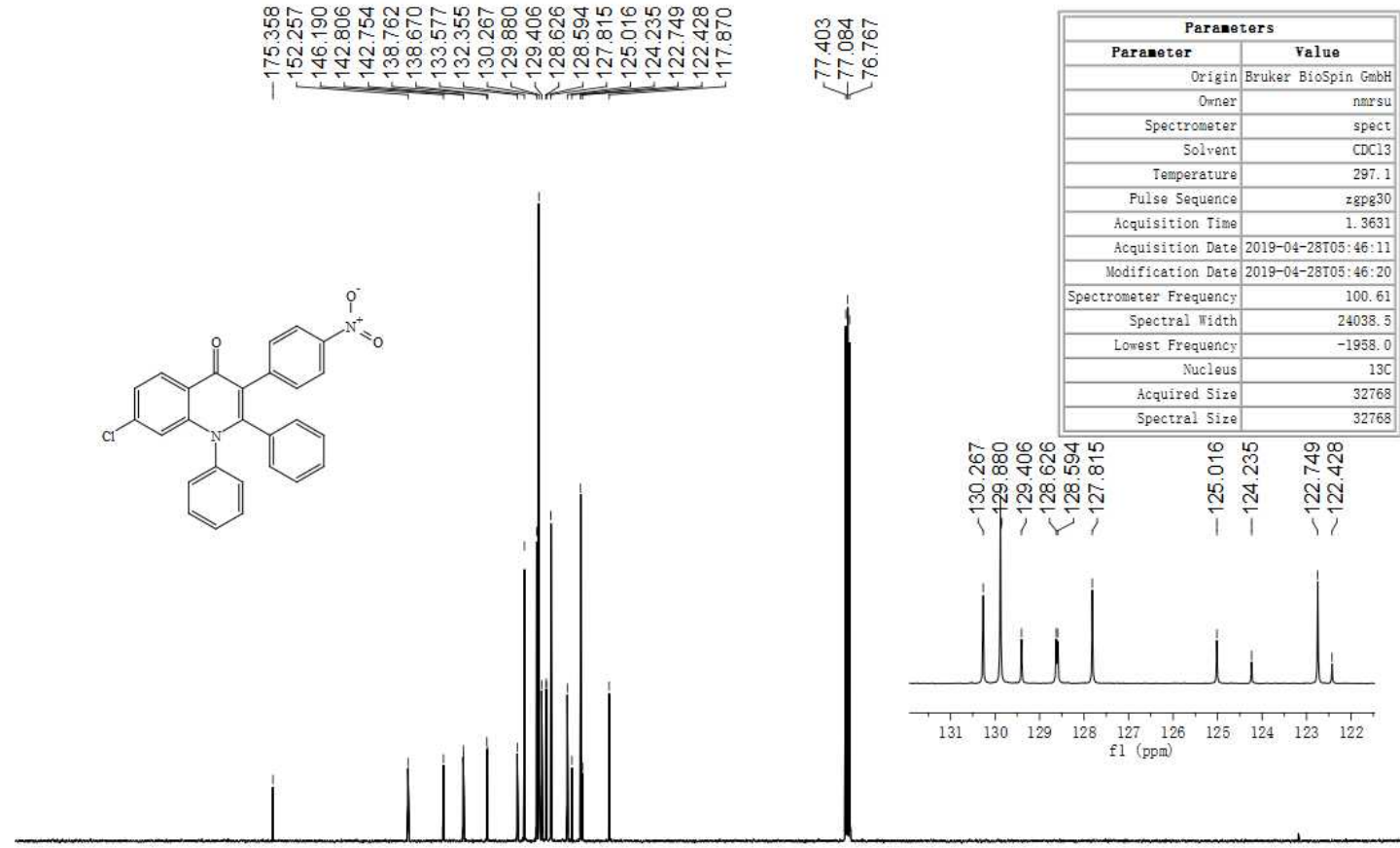

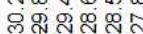

กับ ัำ

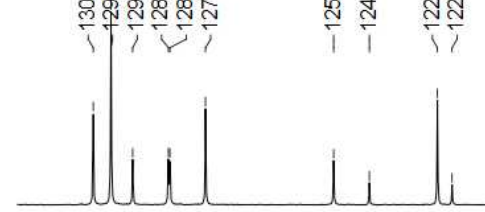

$\begin{array}{llllllllll}131 & 130 & 129 & 128 & \begin{array}{lllll}127 \\ f 1\end{array}(\mathrm{pgm}) & 126 & 124 & 123 & 122\end{array}$

$\begin{array}{lllllllllllll}210 & 200 & 190 & 180 & 170 & 160 & 150 & 140 & 130 & 120 & 110 & 100 & 9\end{array}$ 
7-Fluoro-3-(4-nitrophenyl)-1,2-diphenylquinolin-4(1H)-one (3s)

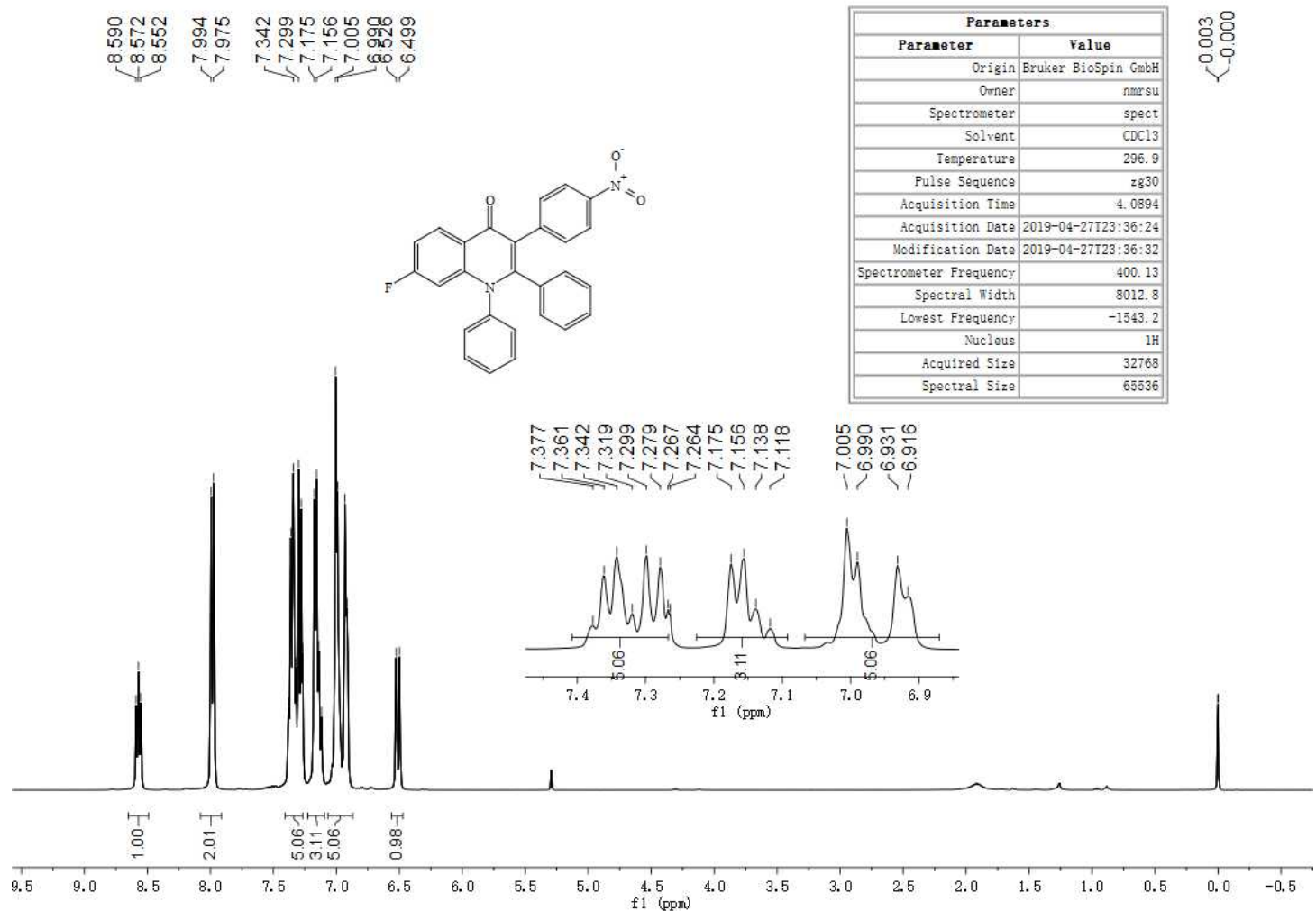

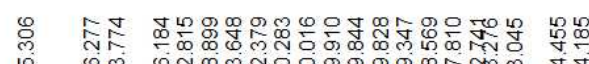

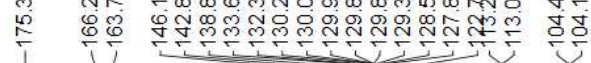
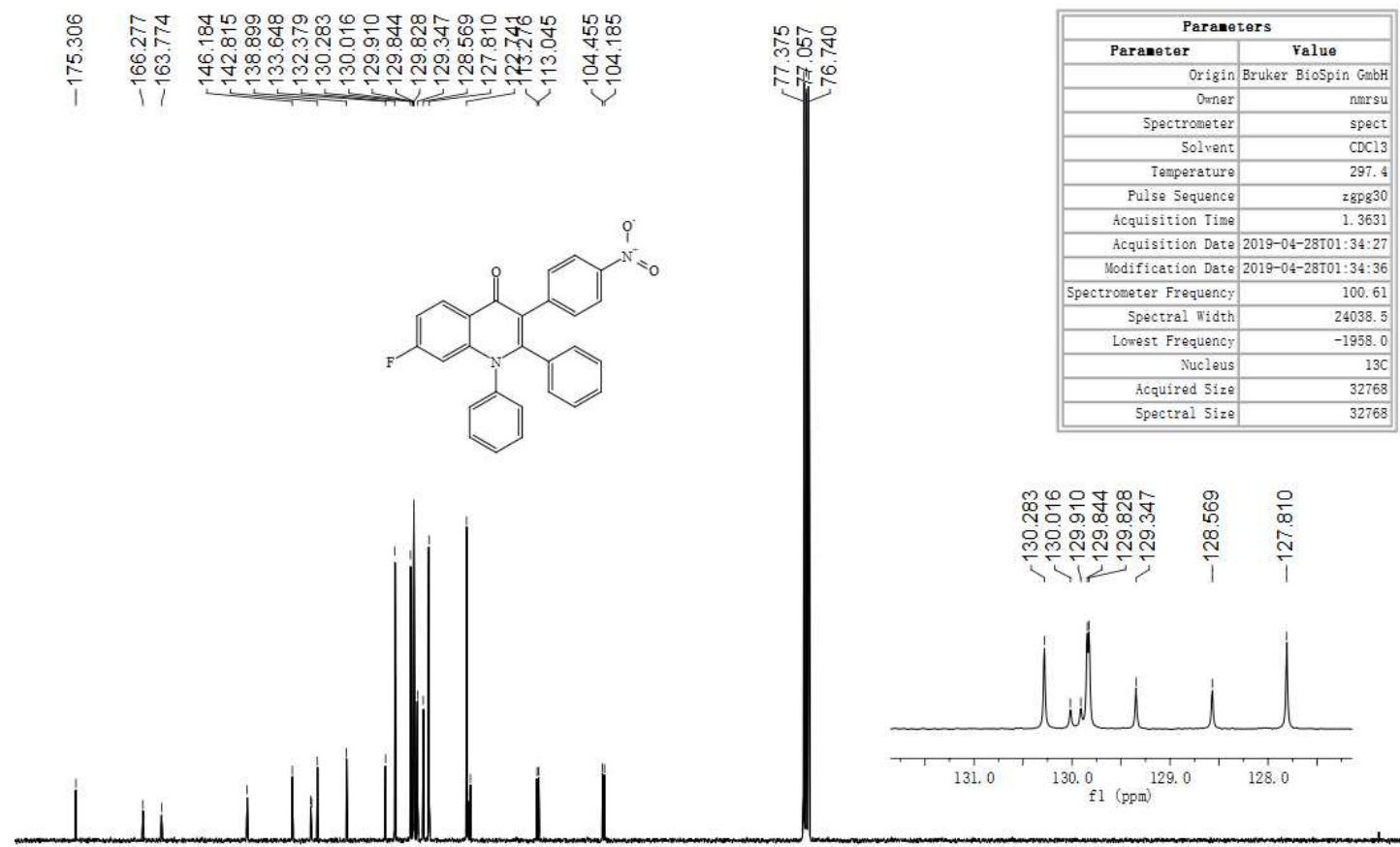

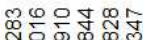

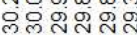

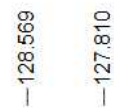

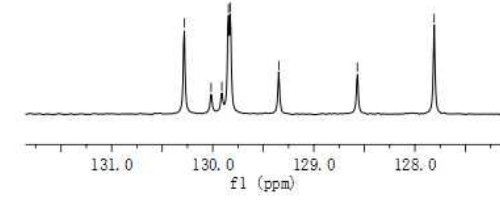




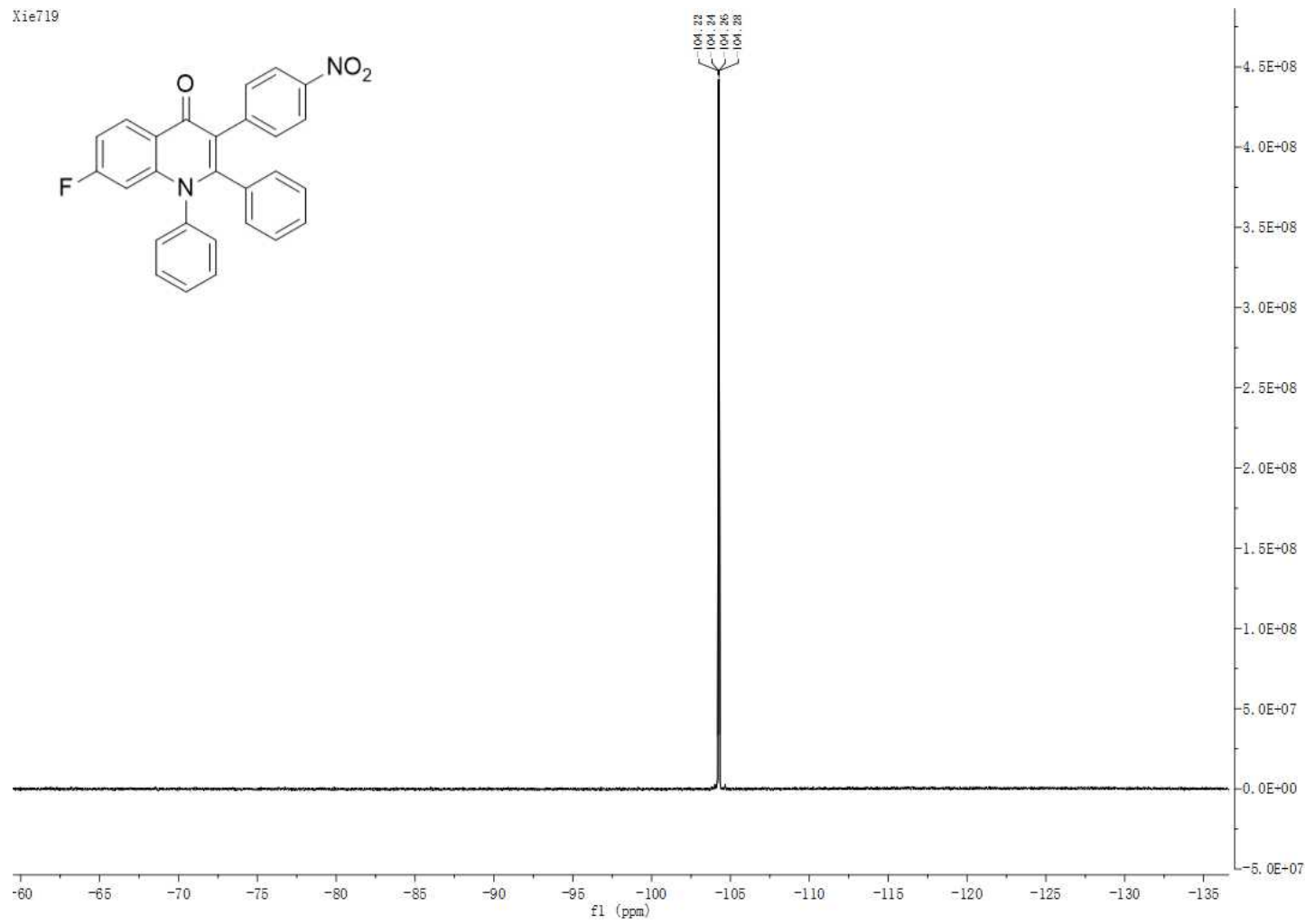

3-(4-Nitrophenyl)-1-phenyl-2-(p-tolyl)quinolin-4(1H)-one (3t)

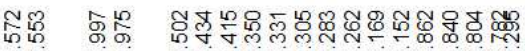

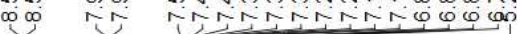

$\stackrel{\substack{0 \\ i}}{i}$

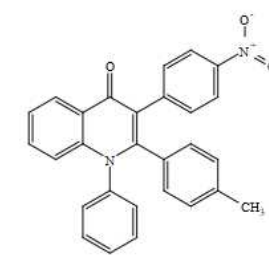

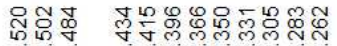

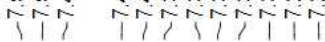
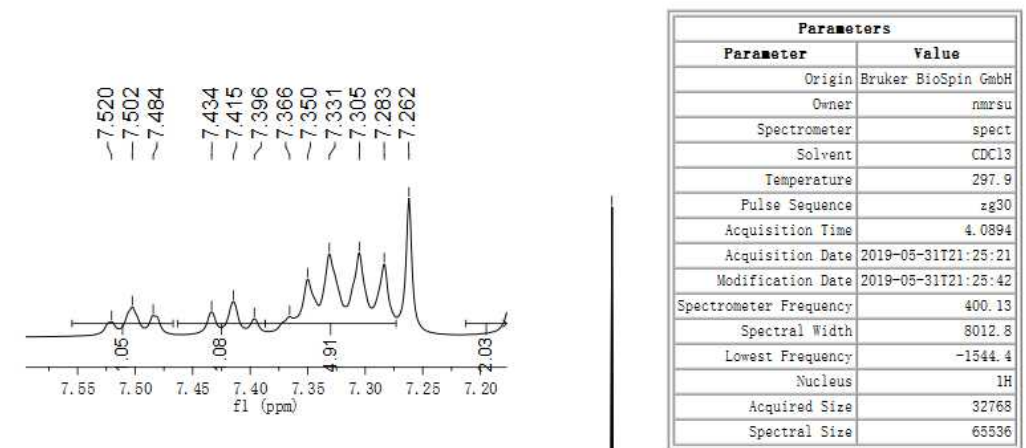

1

65536

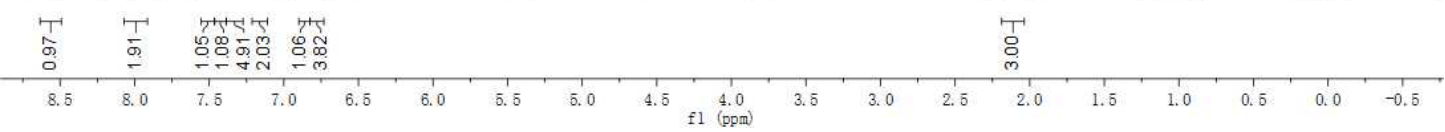



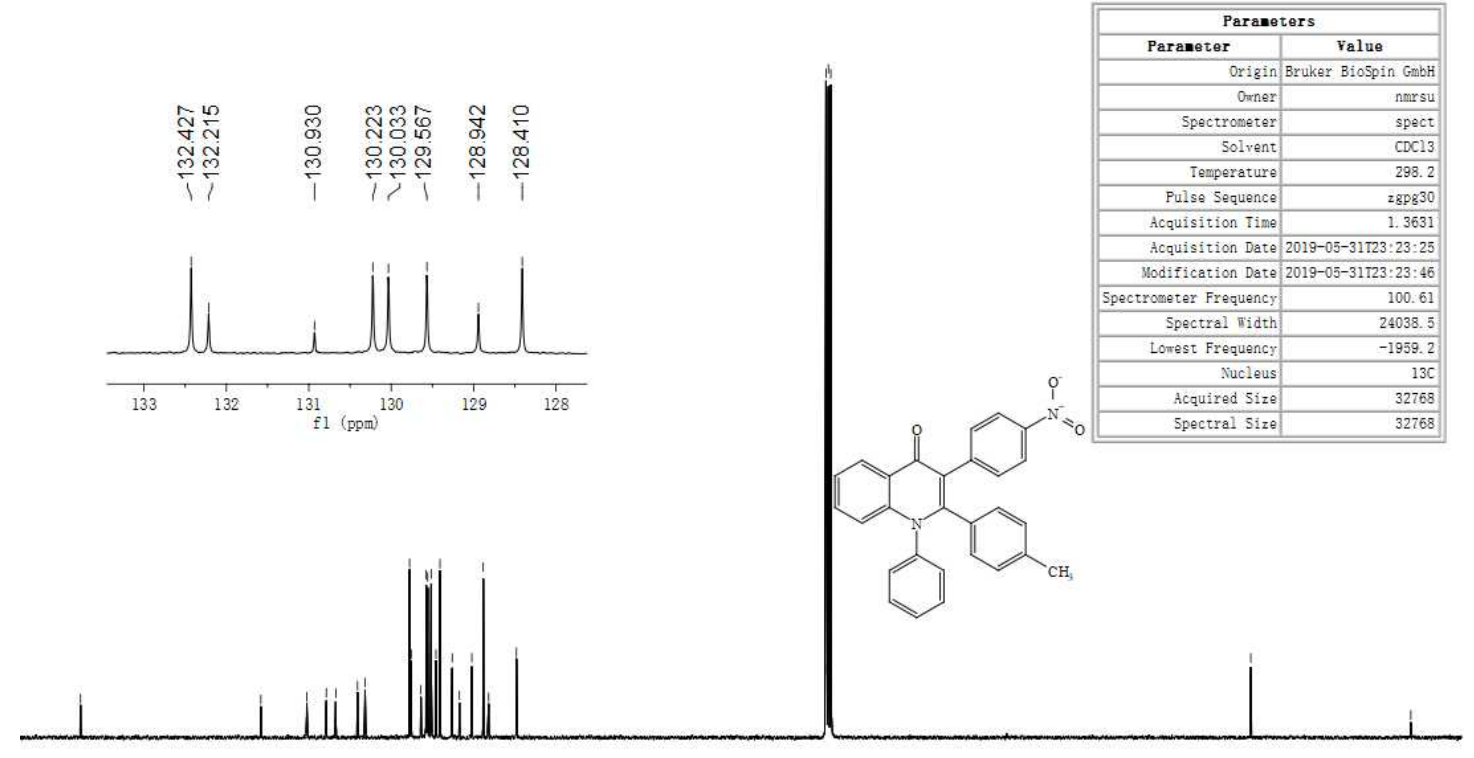

180

$170 \quad 160 \quad 150$

$140 \quad 130$

${ }_{110}^{1}+100$

$9^{1} 80$

2-(4-Methoxyphenyl)-3-(4-nitrophenyl)-1-phenylquinolin-4(1H)-one (3u)

듀유

in

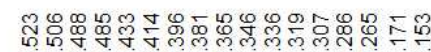

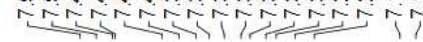
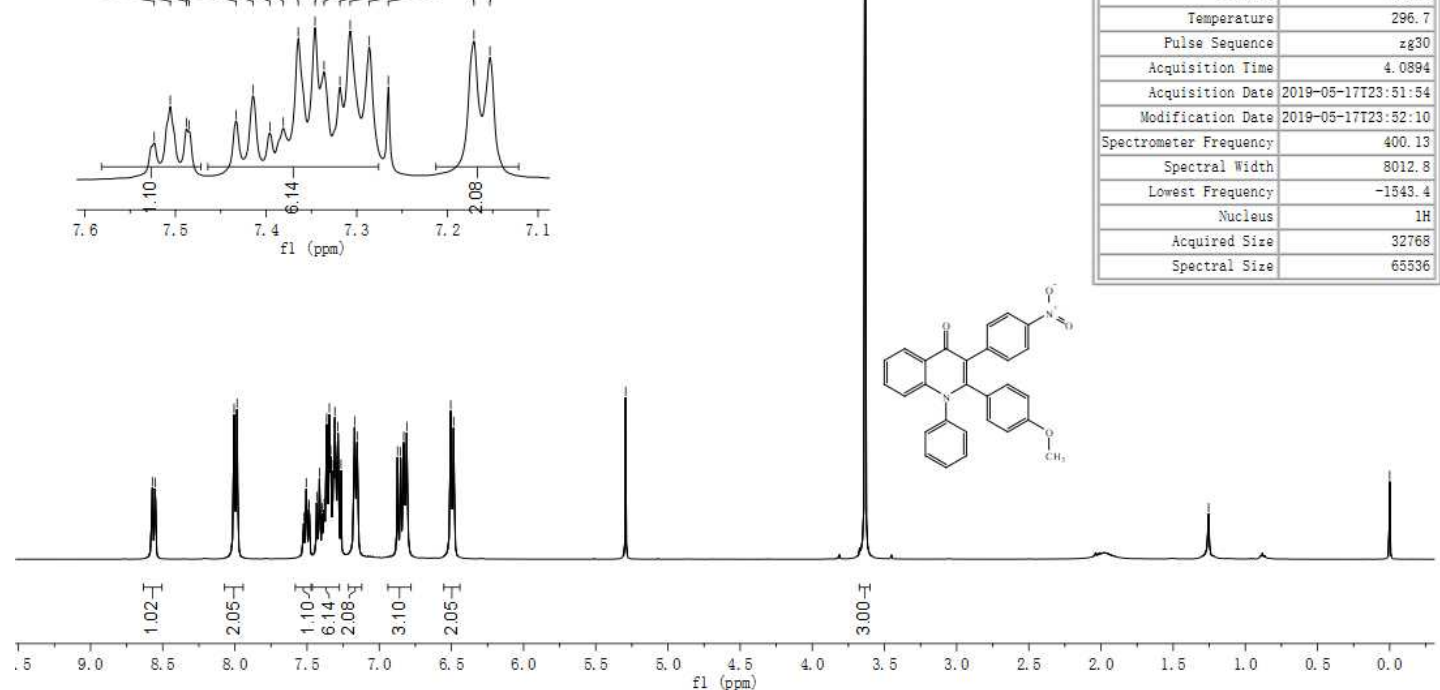


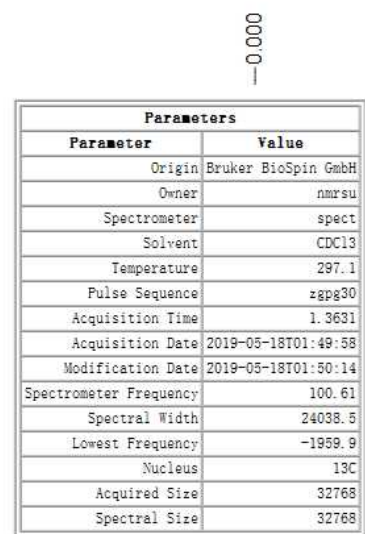

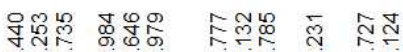
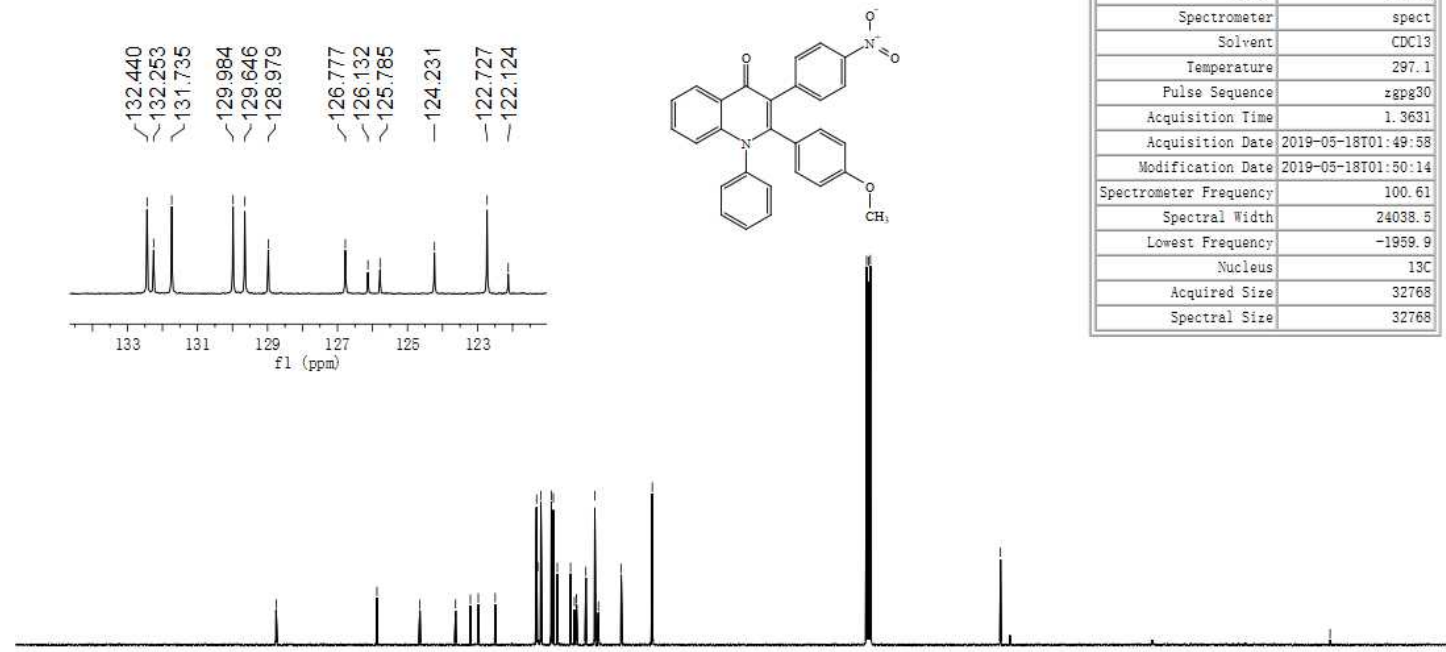

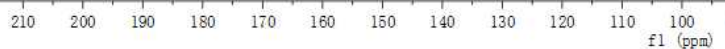

\section{2-(4-Chlorophenyl)-3-(4-nitrophenyl)-1-phenylquinolin-4(1H)-one (3v)}

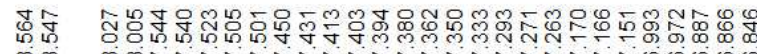

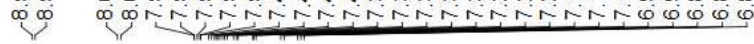

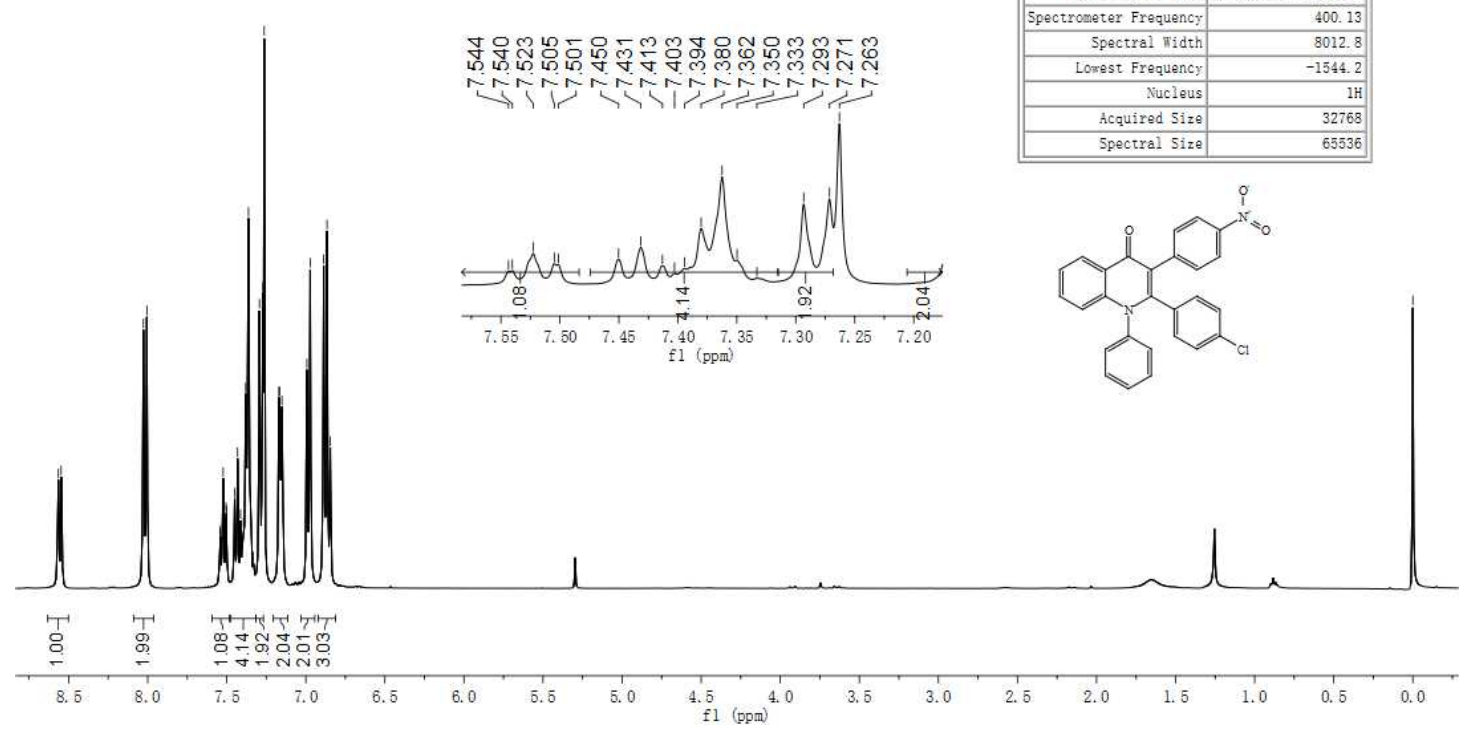




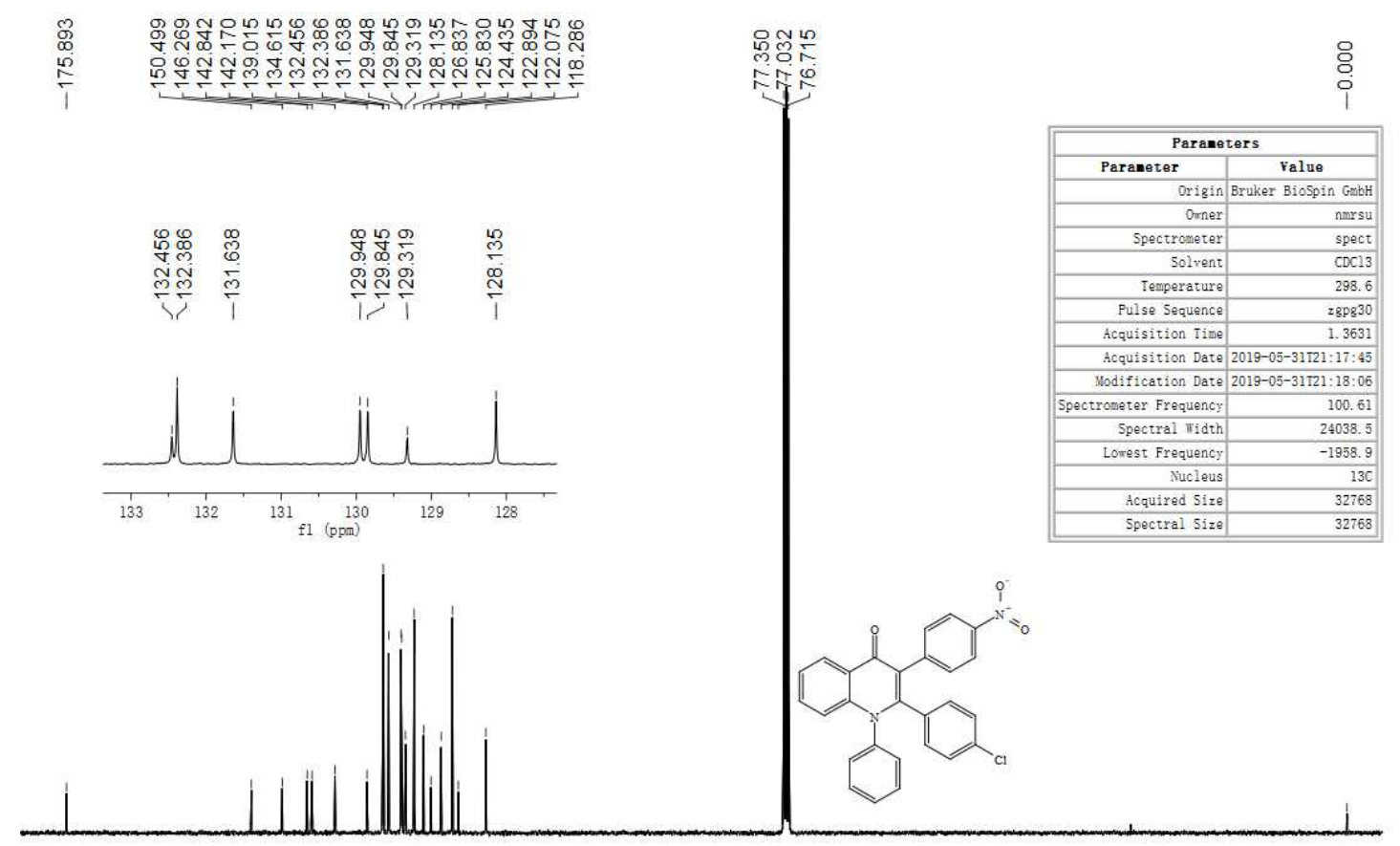

2-(4-Fluorophenyl)-3-(4-nitrophenyl)-1-phenylquinolin-4(1H)-one (3w)

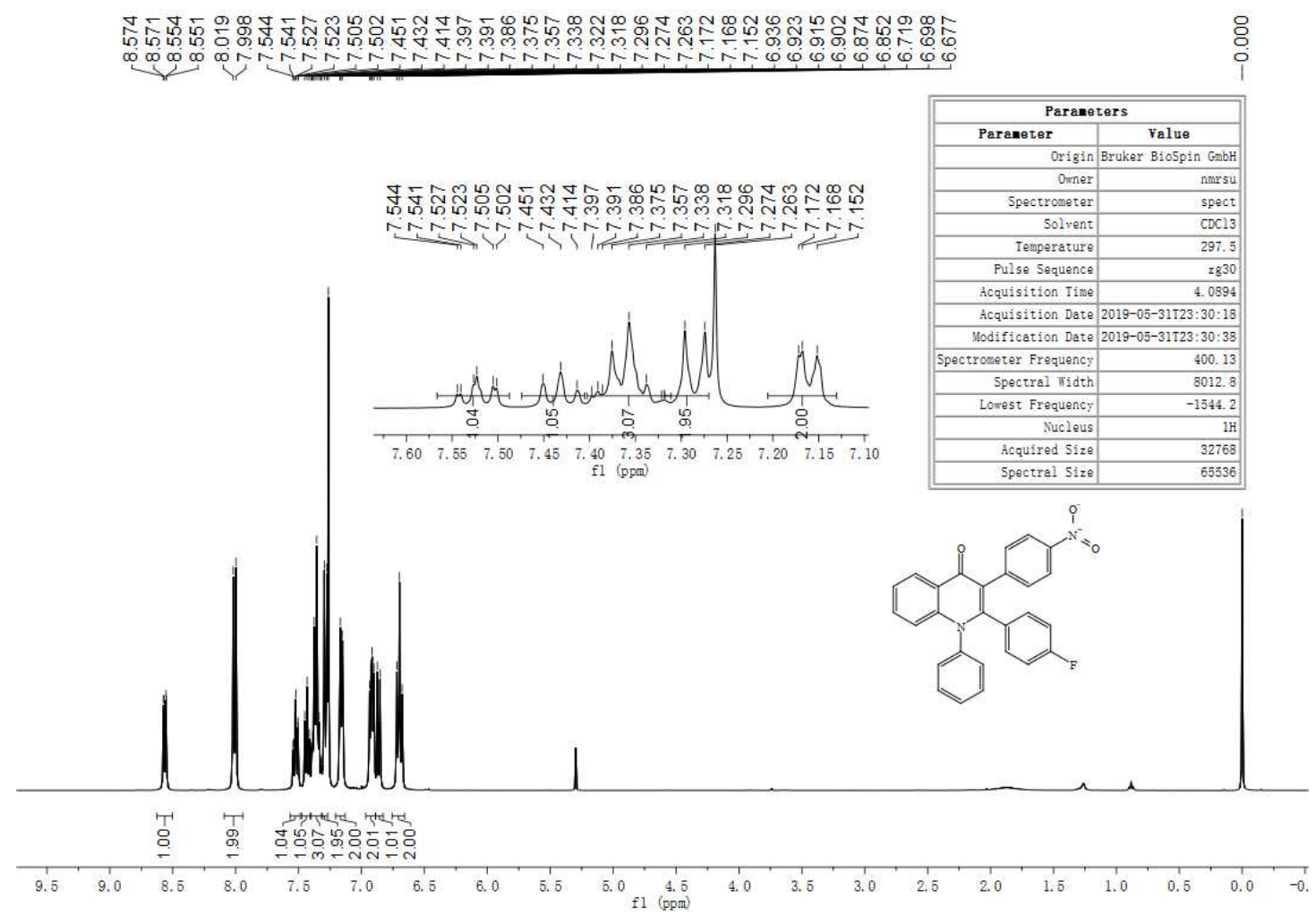




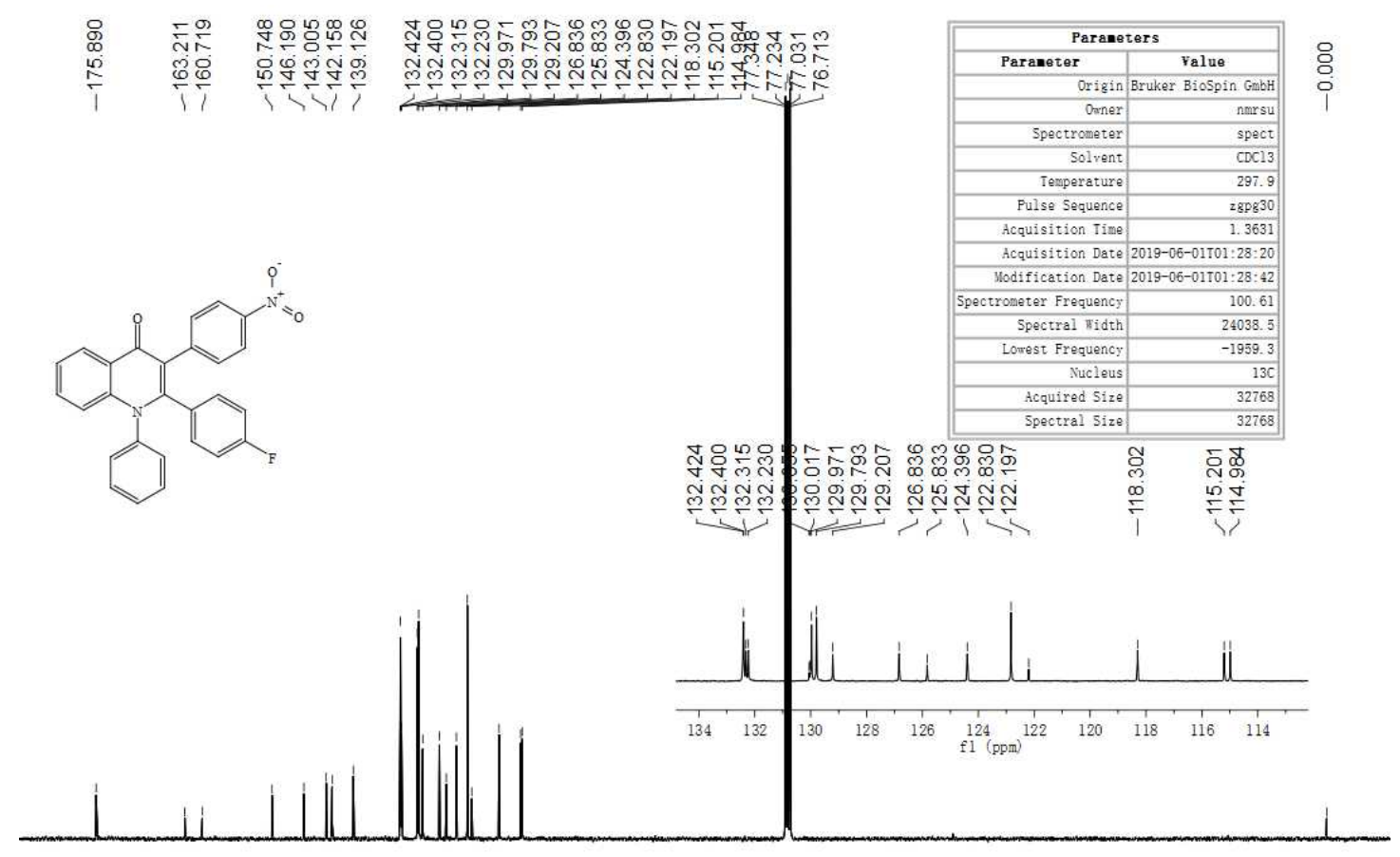

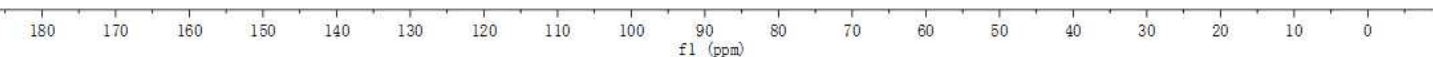




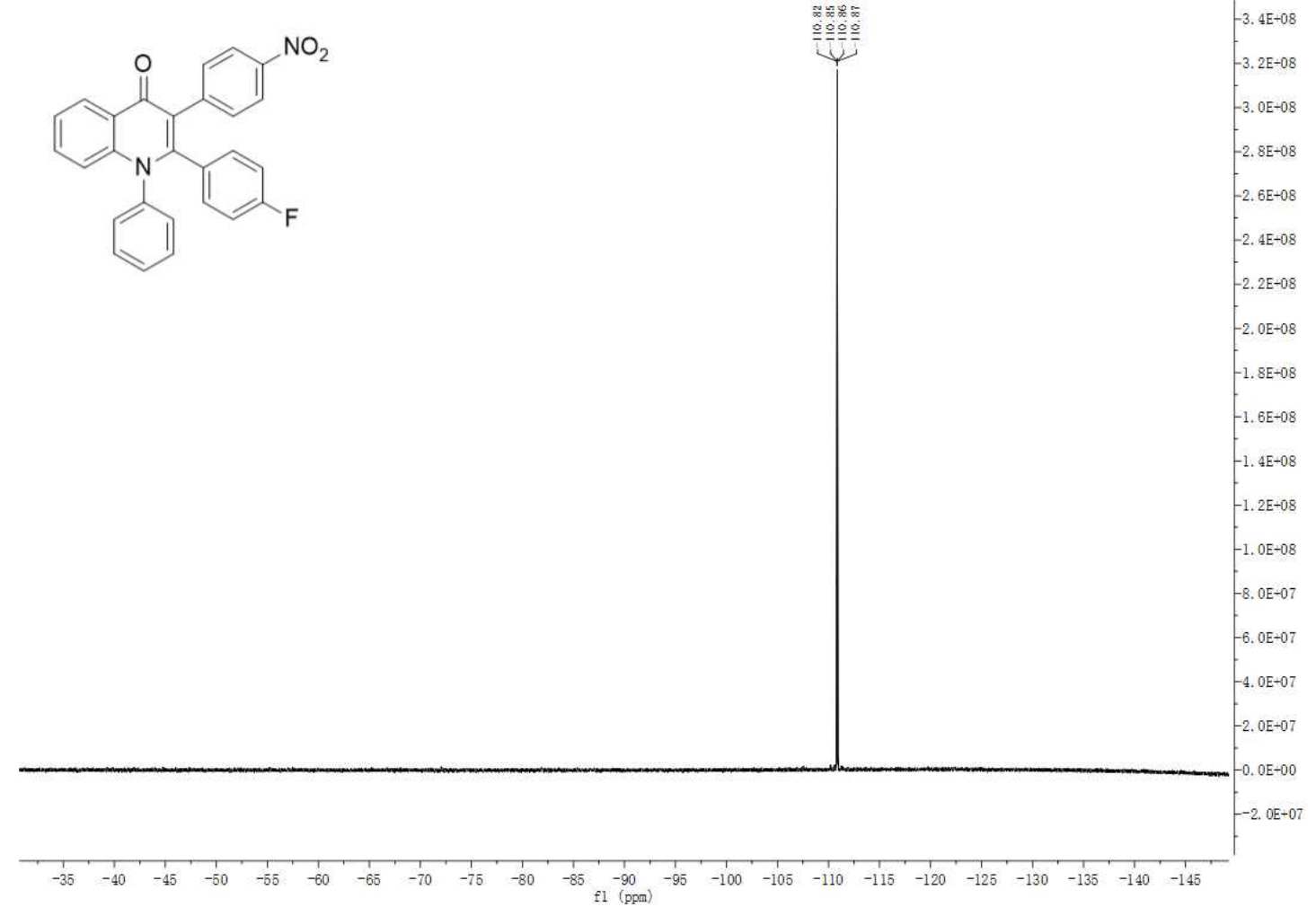

\section{2-(3-Fluorophenyl)-3-(4-nitrophenyl)-1-phenylquinolin-4(1H)-one (3x)}

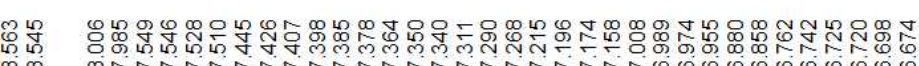

o
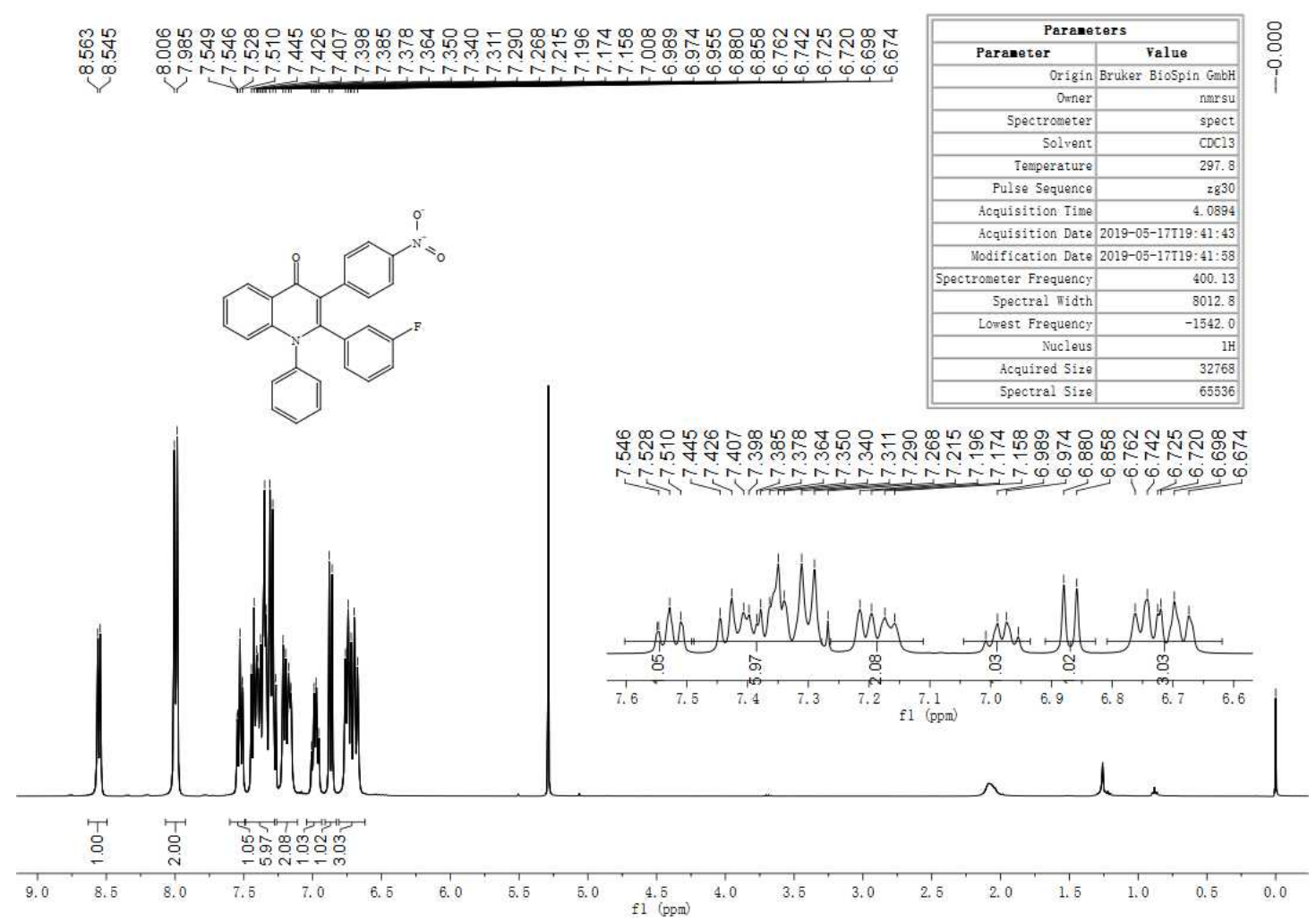


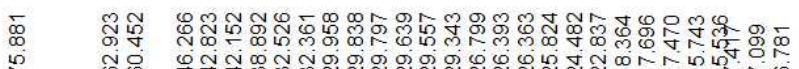

i

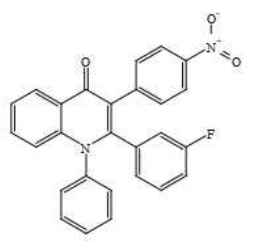

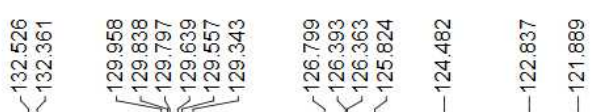

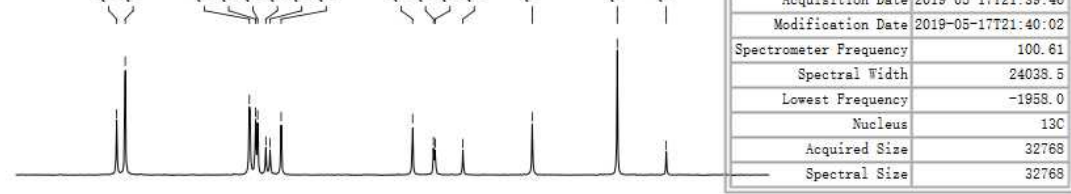

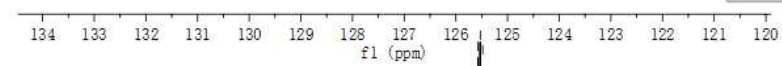

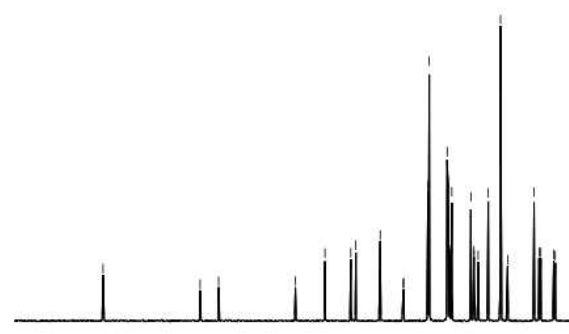

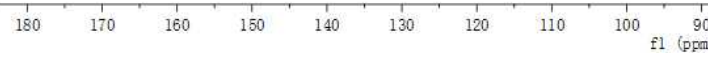

Xie723<smiles>O=c1c(-c2ccc([N+](=O)[O-])cc2)c(-c2cccc(F)c2)n(-c2ccccc2)c2ccccc12</smiles> 


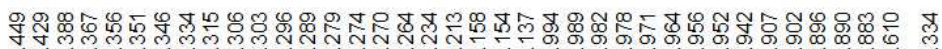
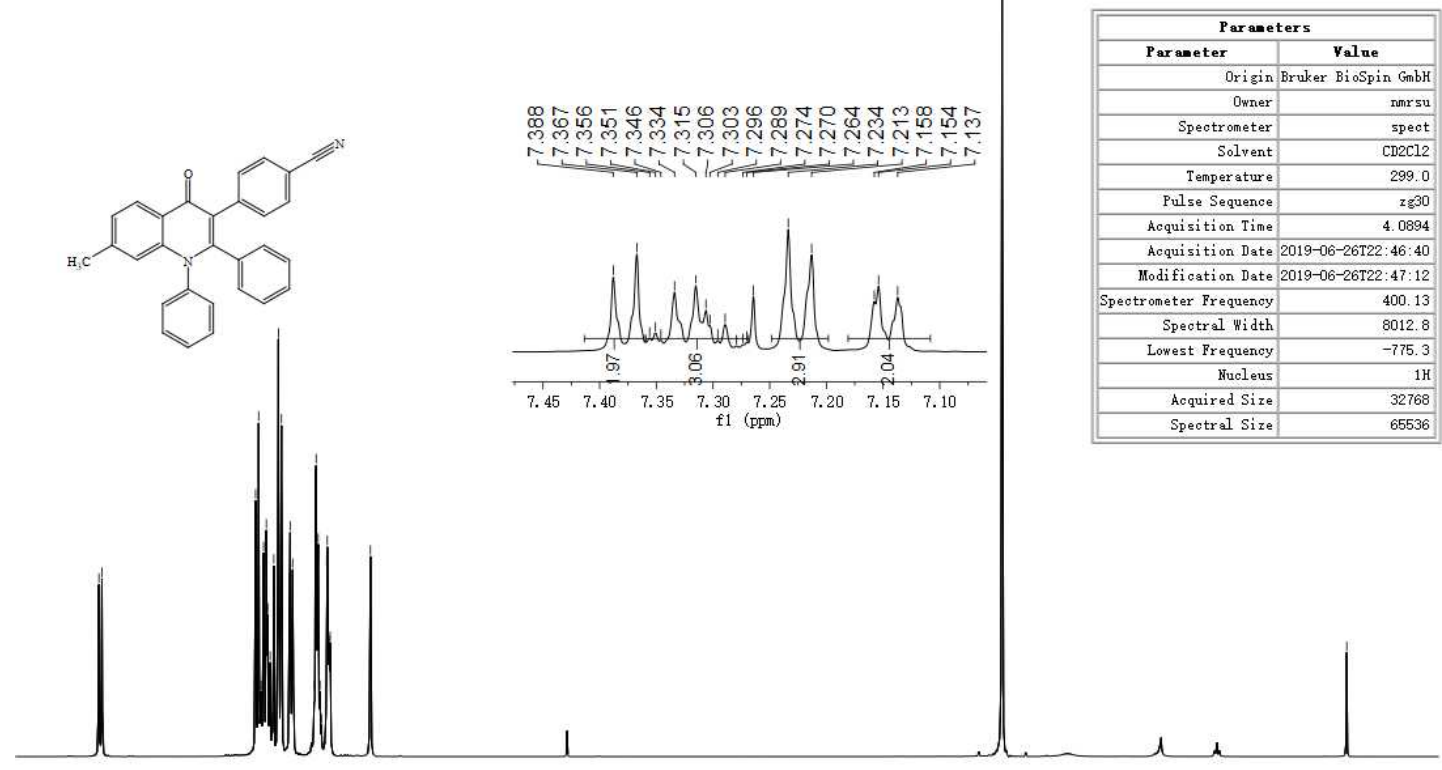

萡
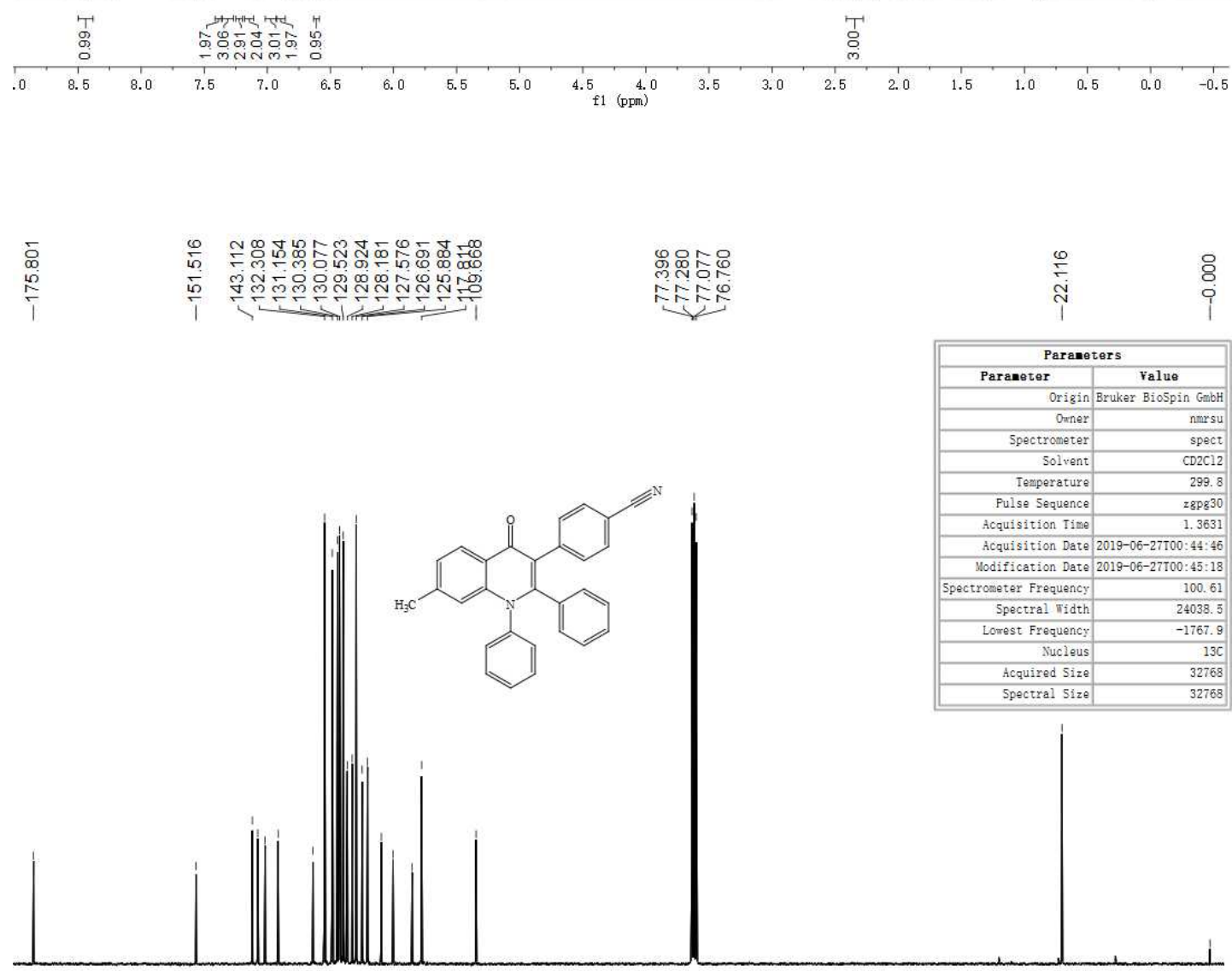

$\begin{array}{lllllllll}170 & 160 & 150 & 140 & 130 & 120 & 110 & 100 & 90 \\ f 1 \text { (ppm) } & 80 & 1\end{array}$ 


\section{4-(7-Fluoro-4-oxo-1,2-diphenyl-1,4-dihydroquinolin-3-yl)benzonitrile (3nb)}

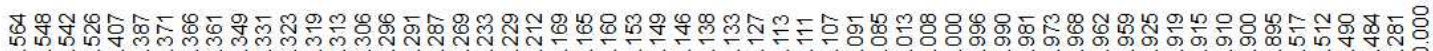

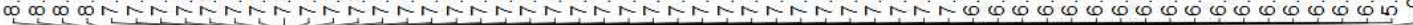<smiles>CC1CCC(C(=O)C(c2ccccc2)C(c2ccccc2)C2CCCCC2)CC1</smiles>

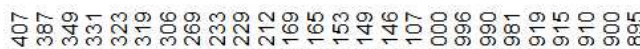

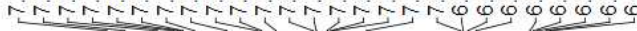
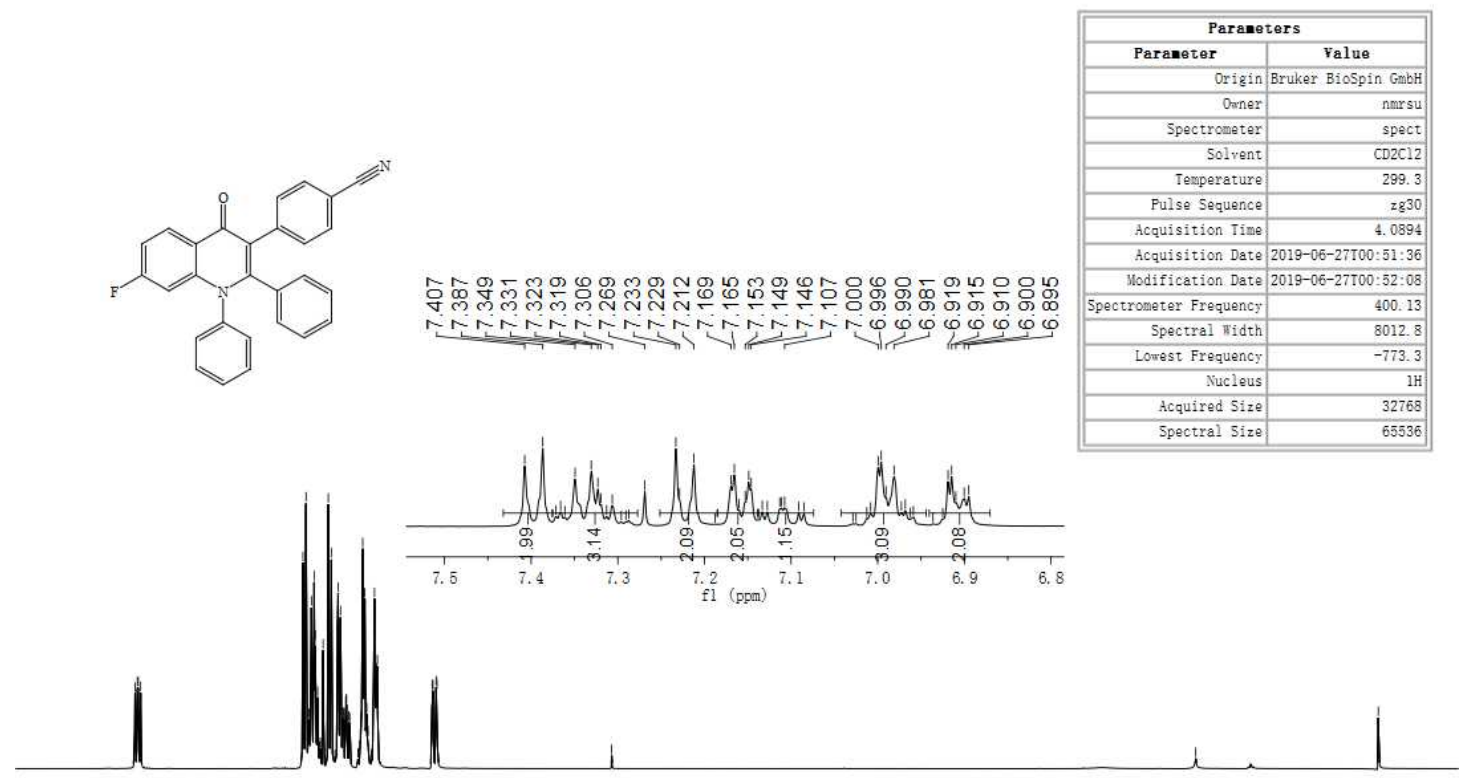

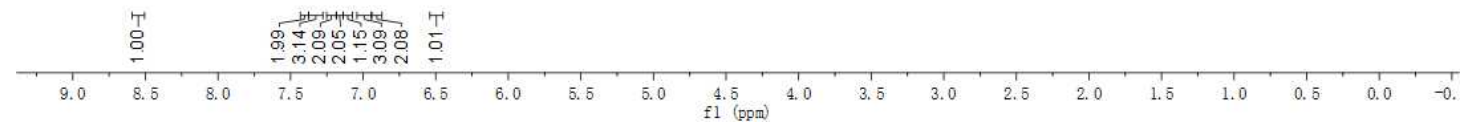
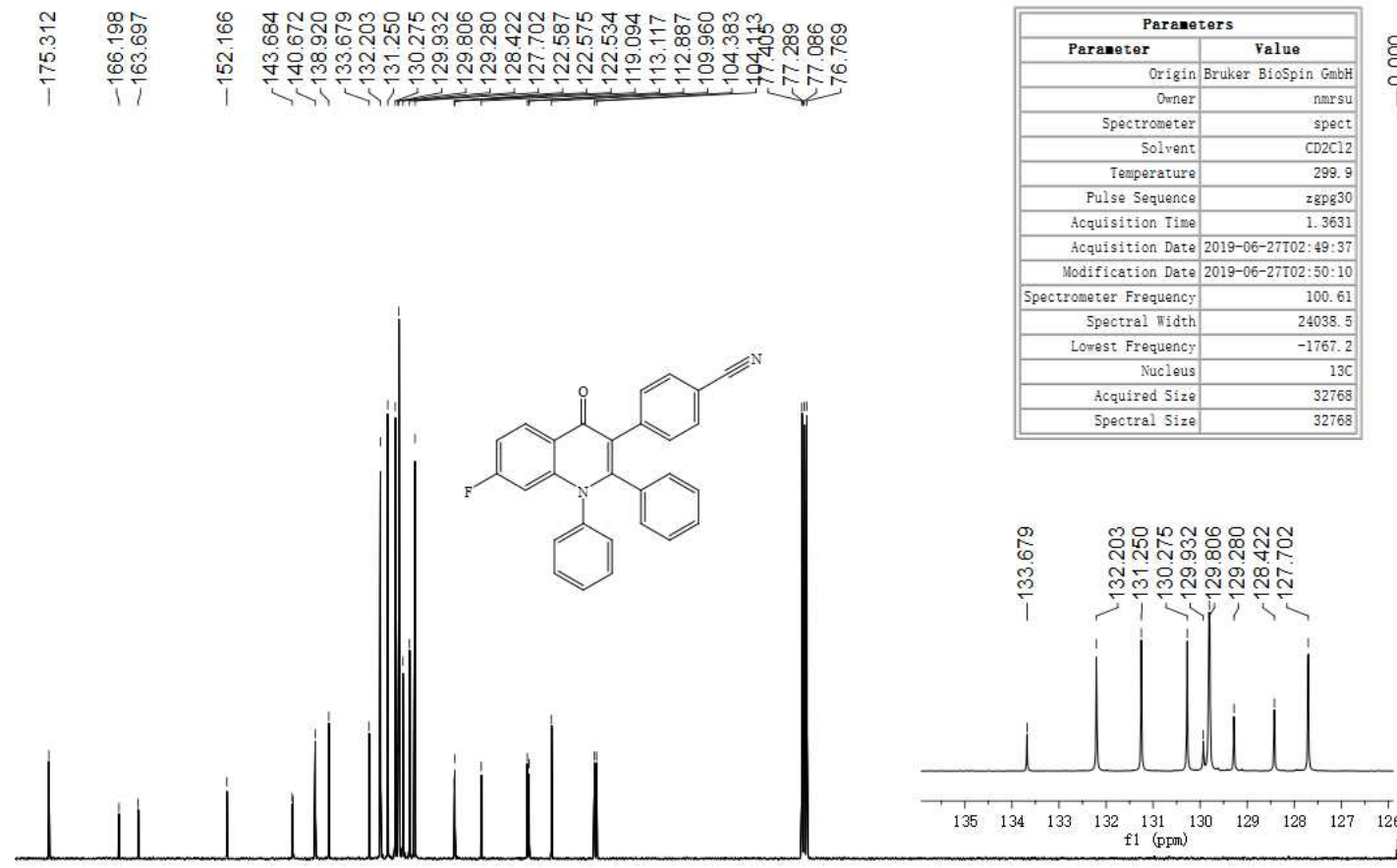

잉
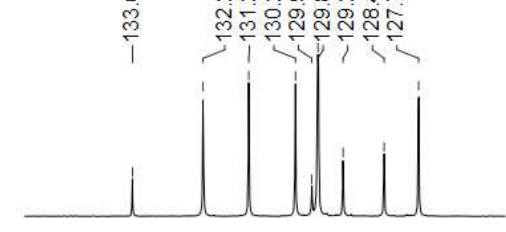

$\begin{array}{lllllllllll}134 & 133 & 132 & 131 & 130 & 129 & 128 & 127 & 126\end{array}$

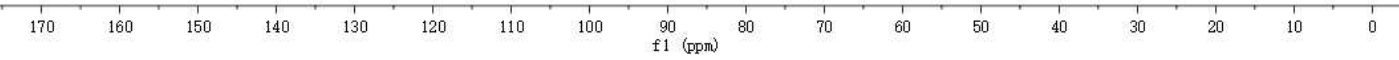




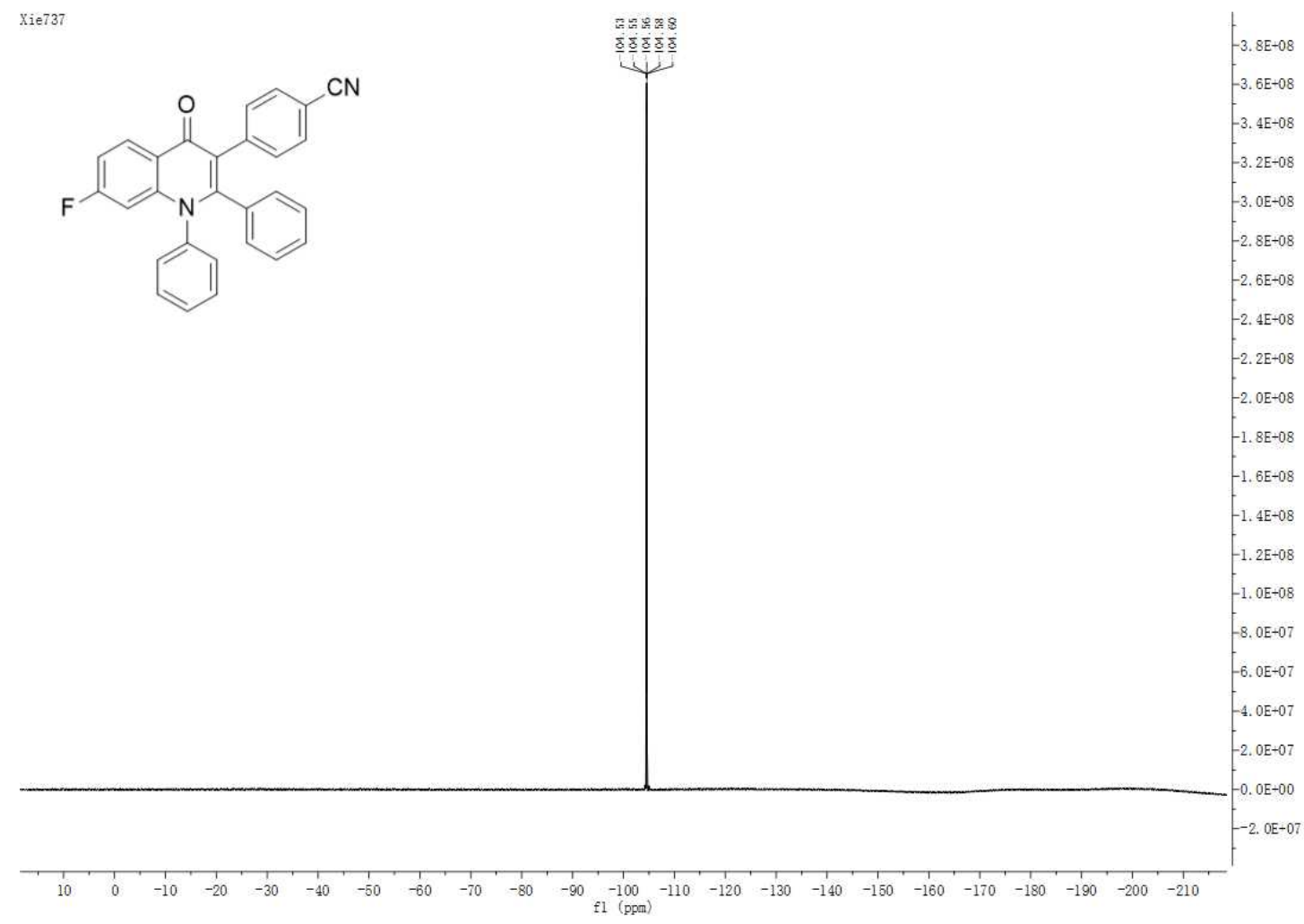

7-Fluoro-3-(2-nitrophenyl)-1,2-diphenylquinolin-4(1H)-one (3oa) 颉品

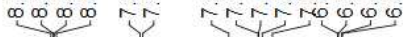

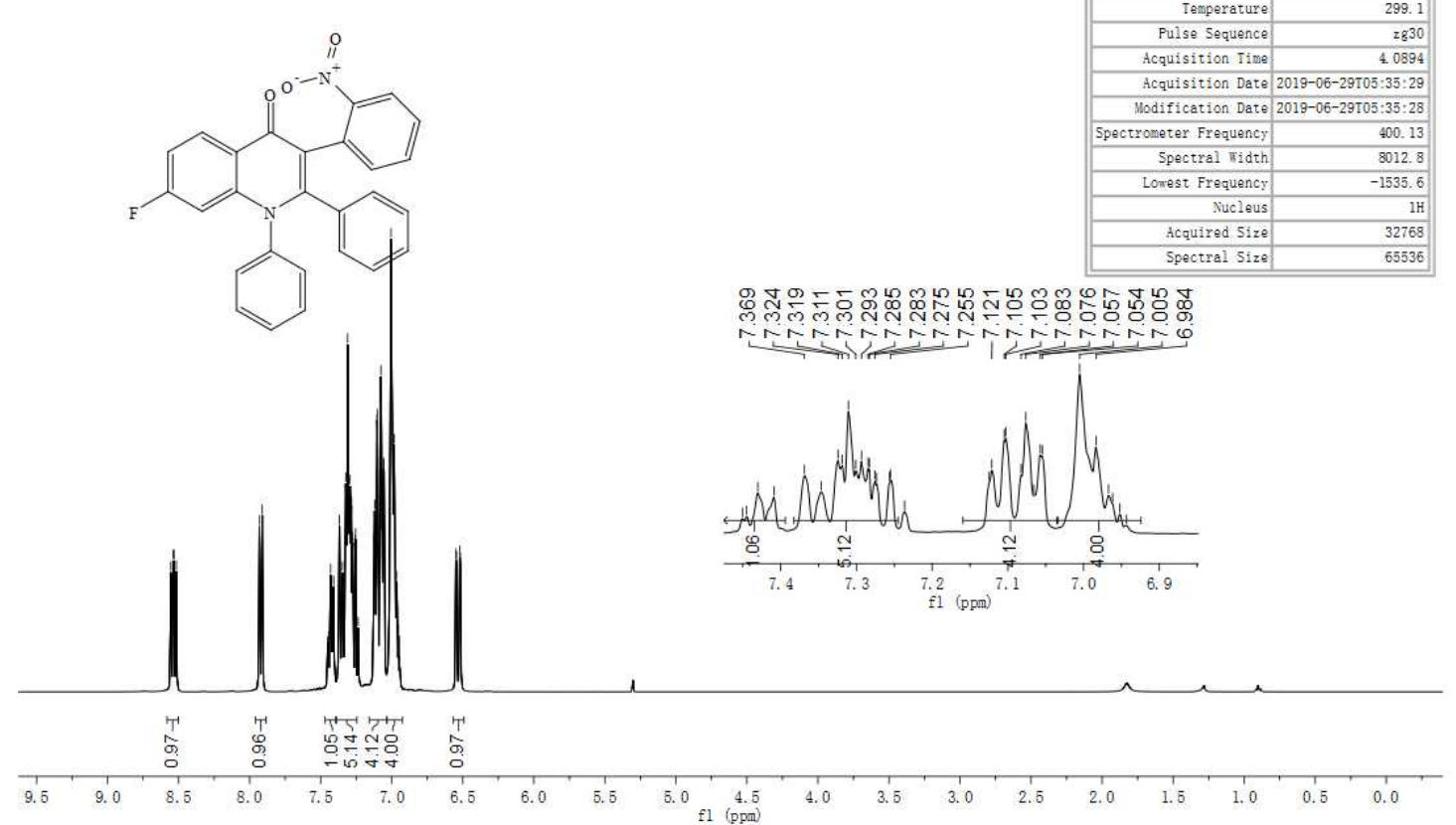




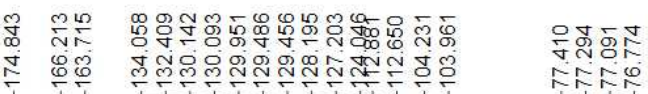

T T 政

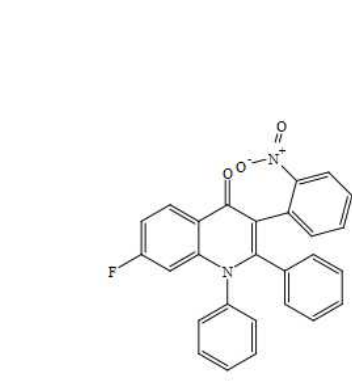

$\begin{array}{llllllllllllll}210 & 200 & 190 & 180 & 170 & 160 & 150 & 140 & 130 & 120 & 110 & 100 \\ \mathrm{f} 1(\mathrm{ppm}) & 90\end{array}$

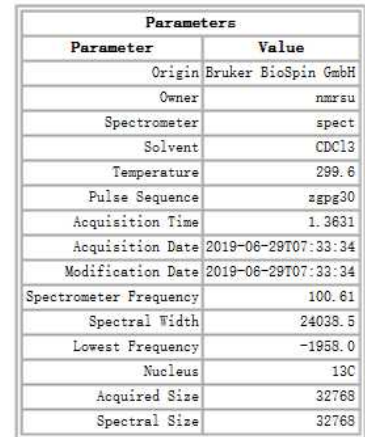

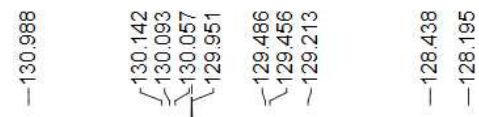

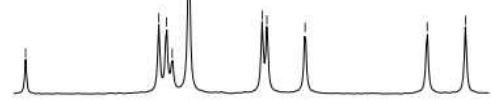

$\begin{array}{rrrrrr}131.0 & 130.5 & 130.0 & 129.5 & 129.0 & 128.5\end{array}$<smiles>O=c1c(-c2ccccc2)c(-c2ccccc2)n(-c2ccccc2)c2cc(F)ccc12</smiles> 
7-Chloro-3-(2-nitrophenyl)-1,2-diphenylquinolin-4(1H)-one (3ob)

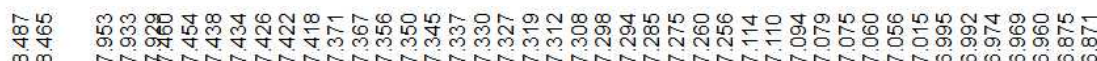

क
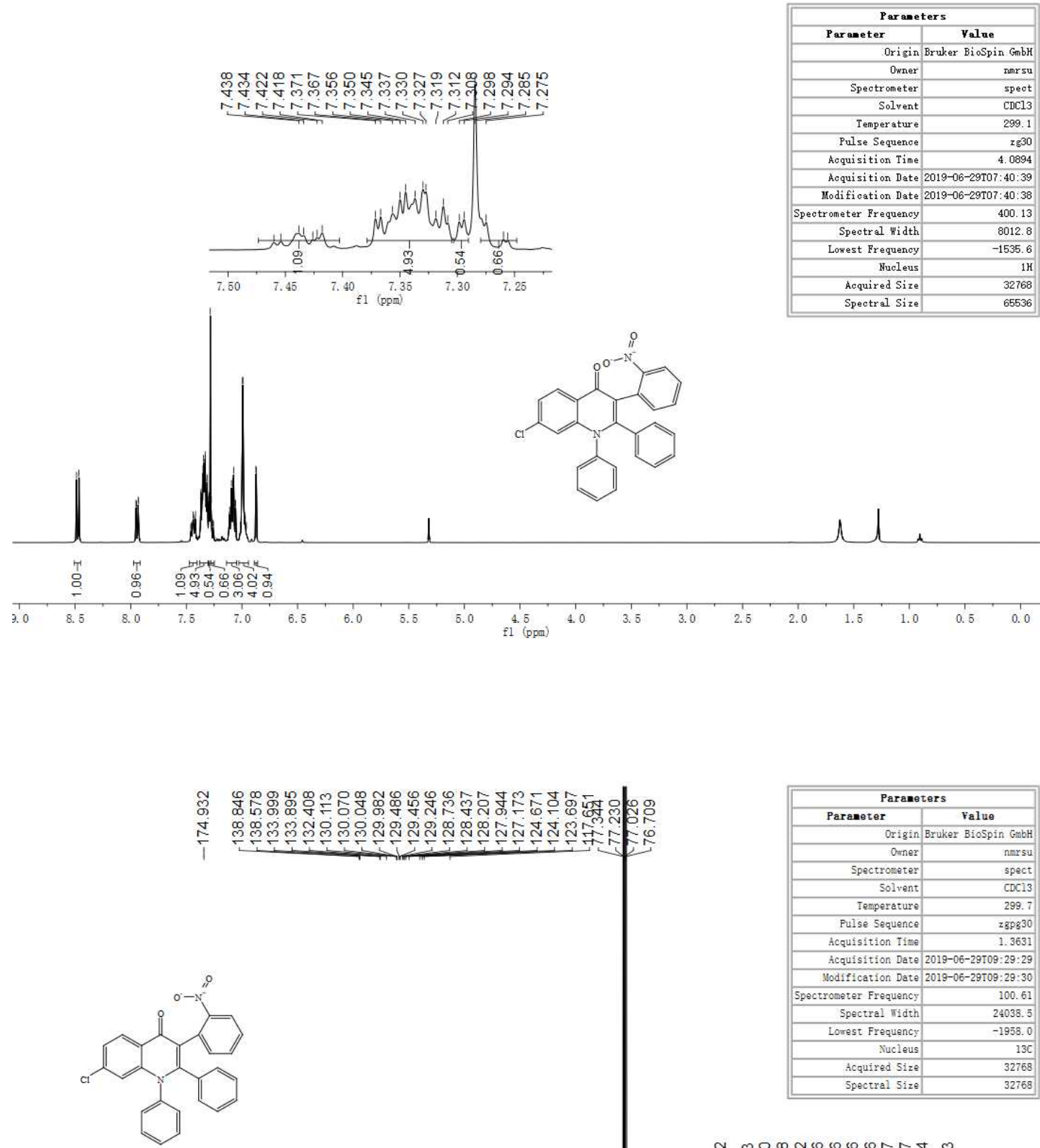

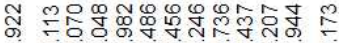

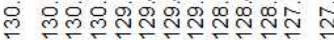

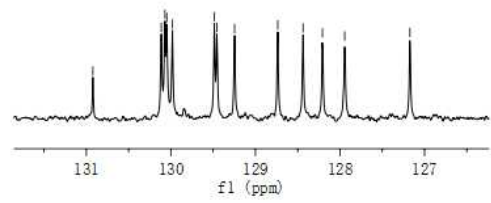

$\begin{array}{llllllllllll}210 & 200 & 190 & 180 & 170 & 160 & 150 & 140 & 130 & 120 & 110 & 100 \\ \mathrm{fl}(\mathrm{ppm}) & \end{array}$ 
2-(4-Fluorophenyl)-3-(2-nitrophenyl)-1-phenylquinolin-4(1H)-one (3oc)

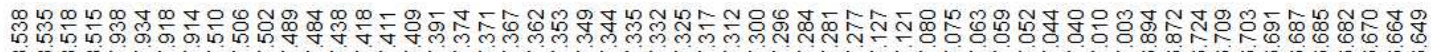

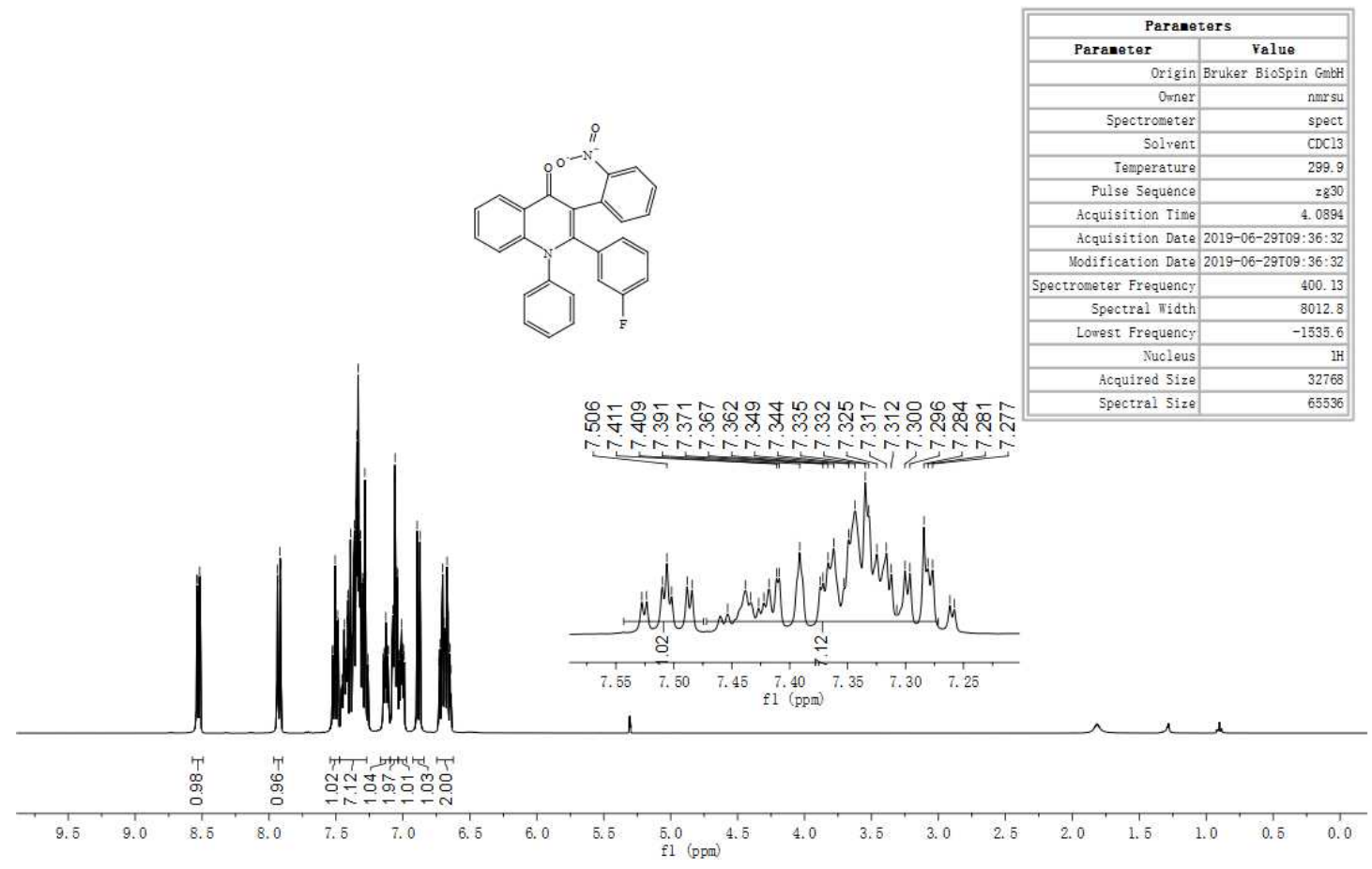

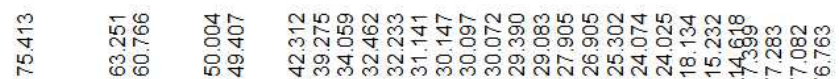

א.
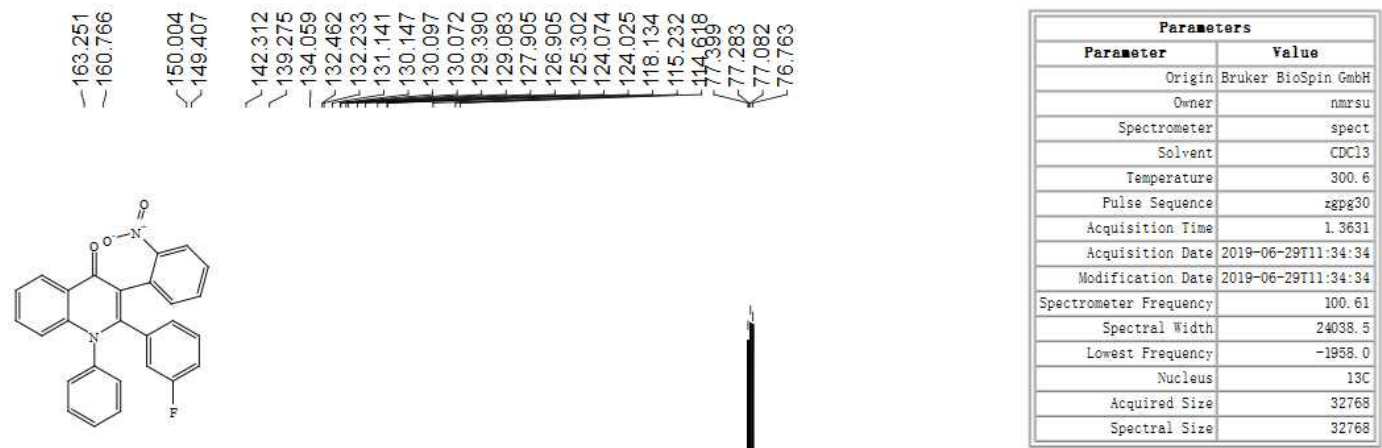

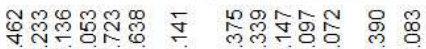
रำ
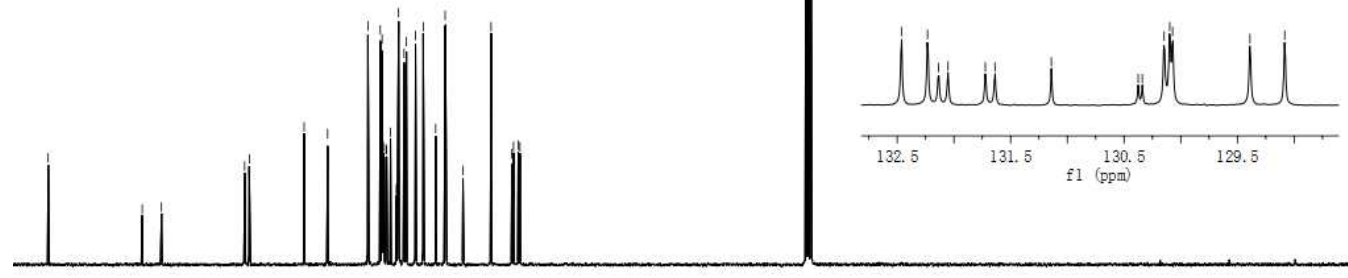

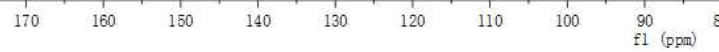




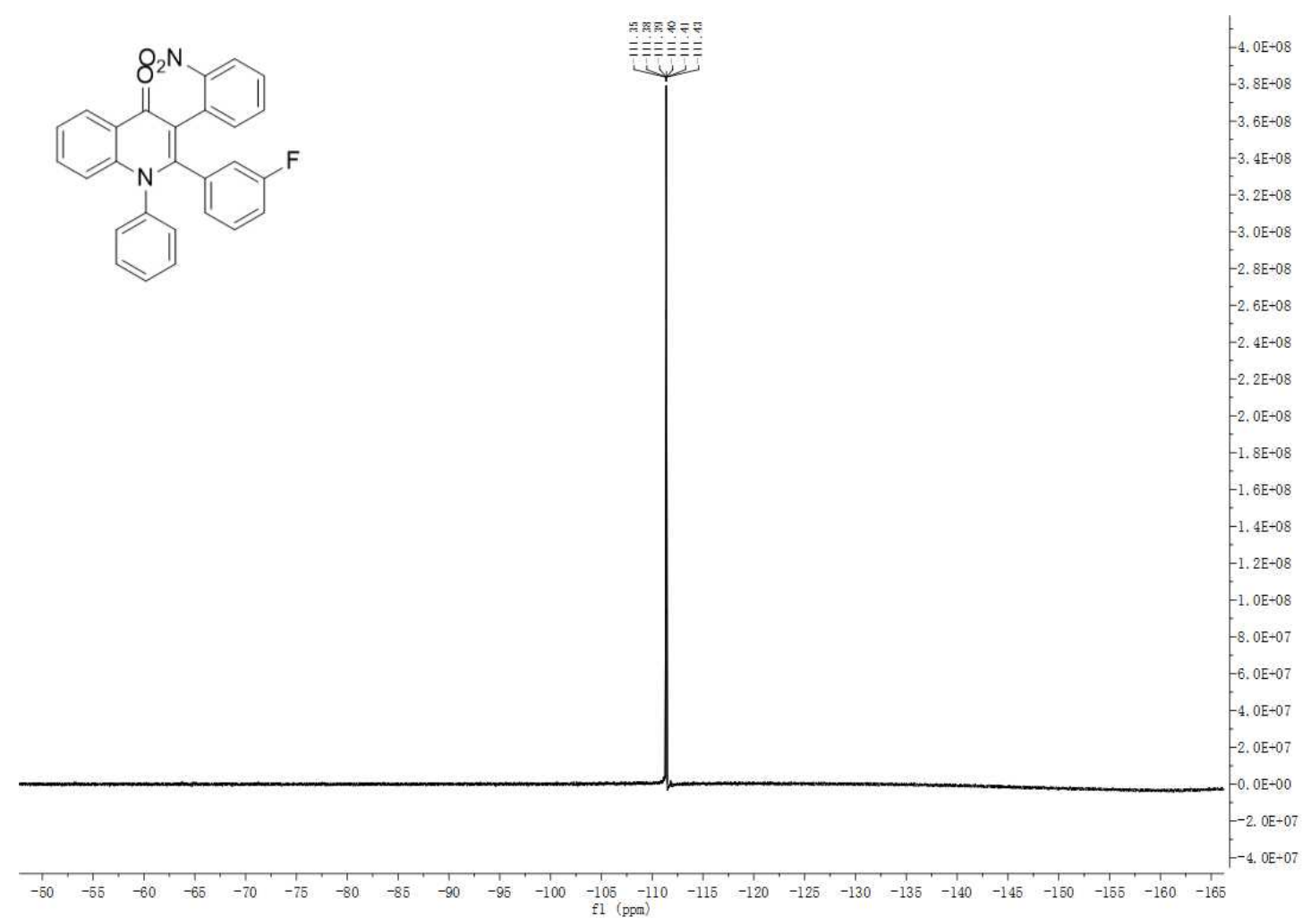

3-(2-Nitrophenyl)-1-phenyl-2-(p-tolyl)quinolin-4(1H)-one (3od)

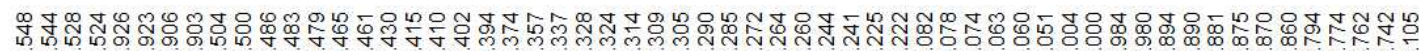

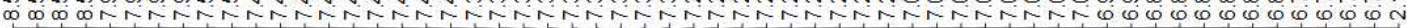
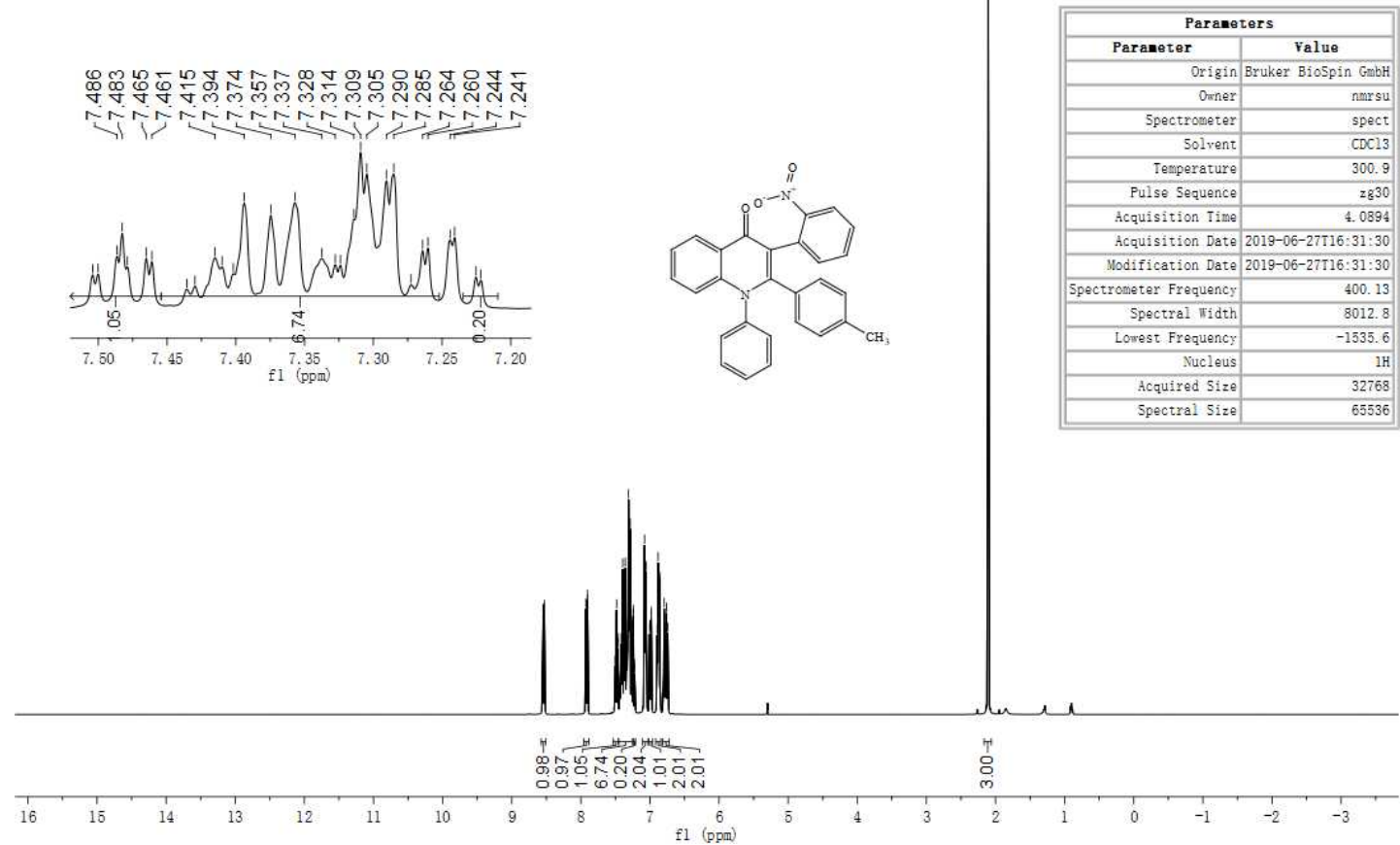


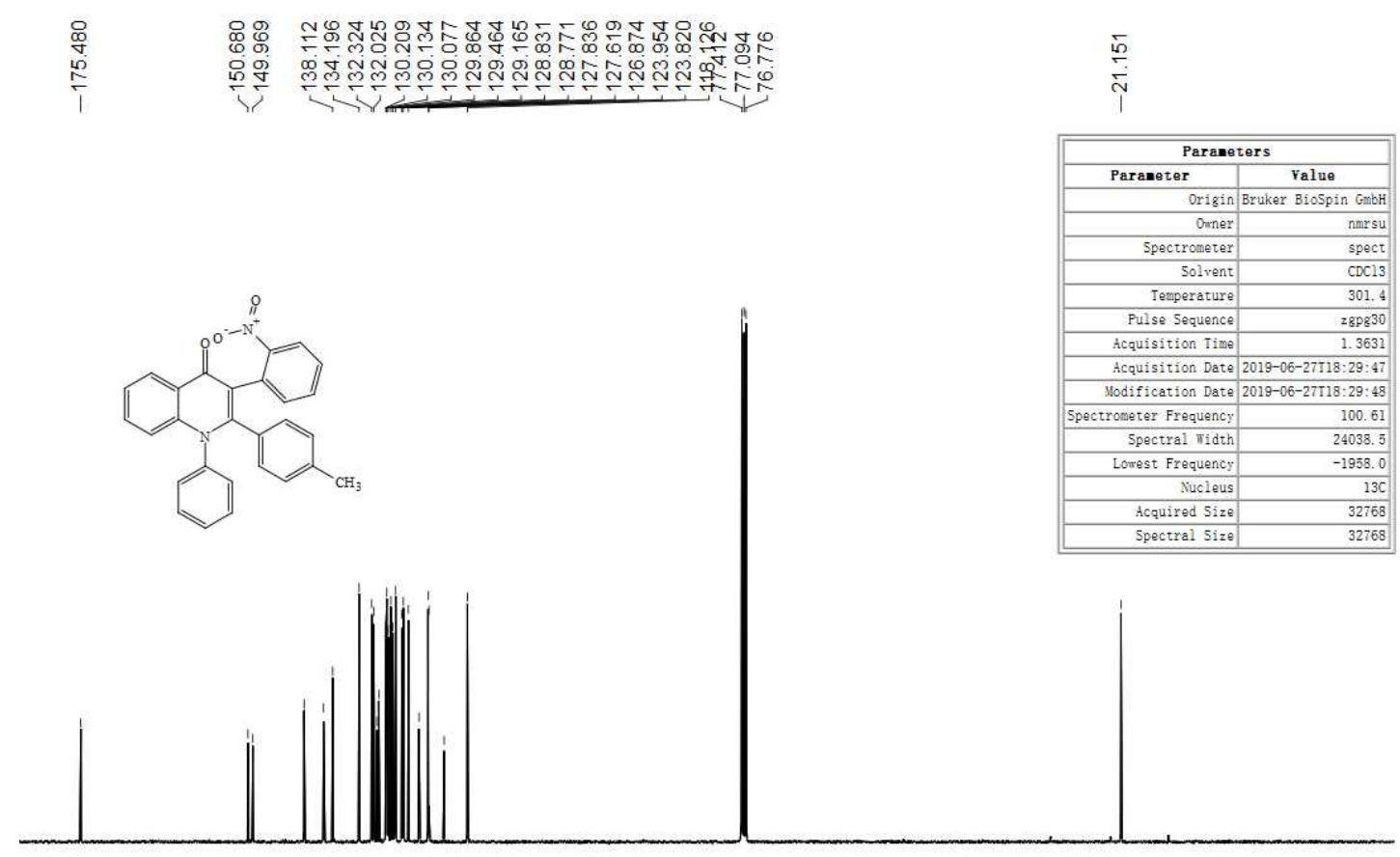

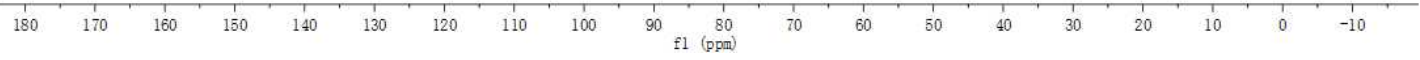

3-(4-Nitrophenyl)-1-(p-tolyl)quinolin-4(1H)-one (3ba/3bb)

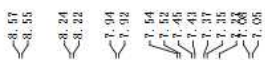

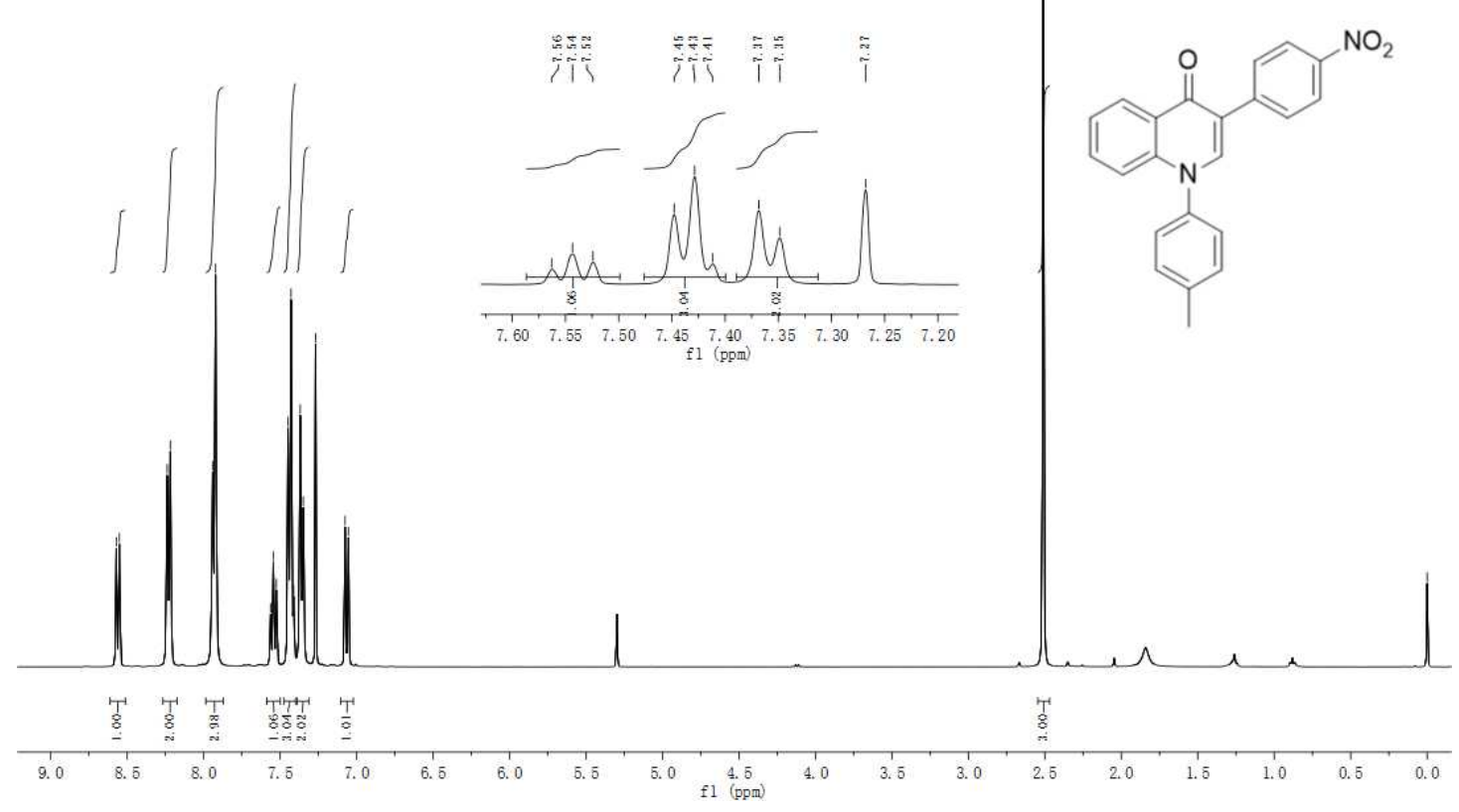



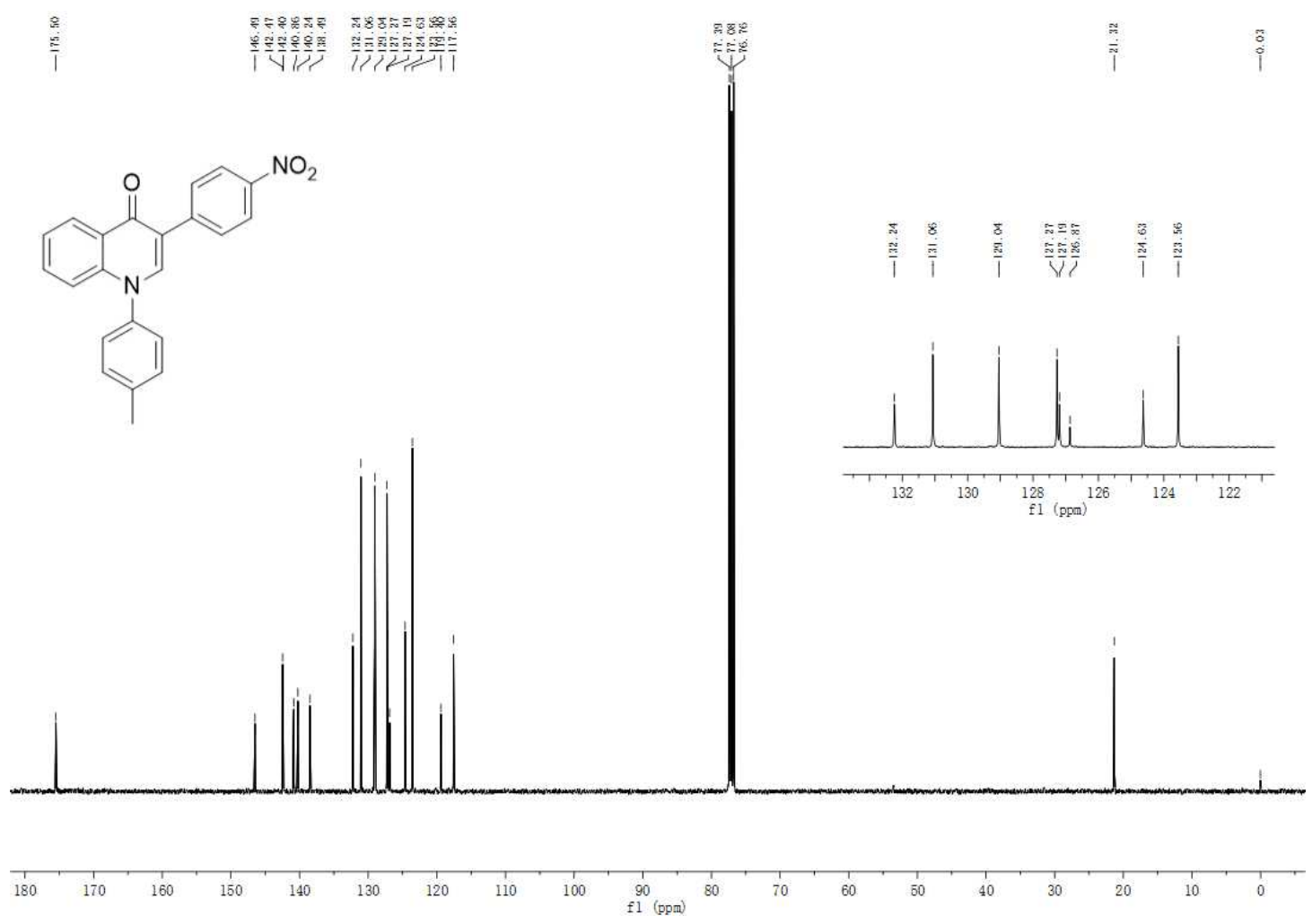

2-methyl-3-(4-nitrophenyl)-1-(p-tolyl)quinolin-4(1H)-one (3bc)

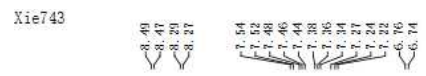

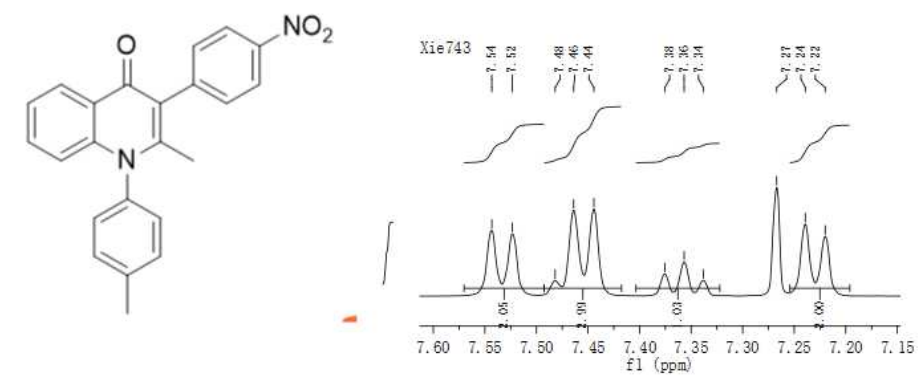

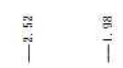

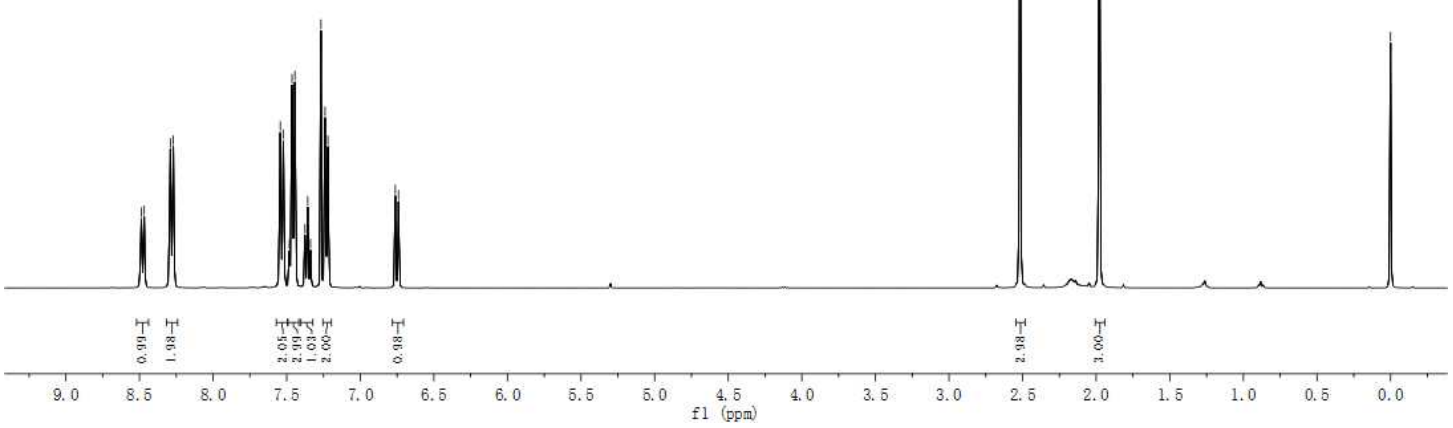




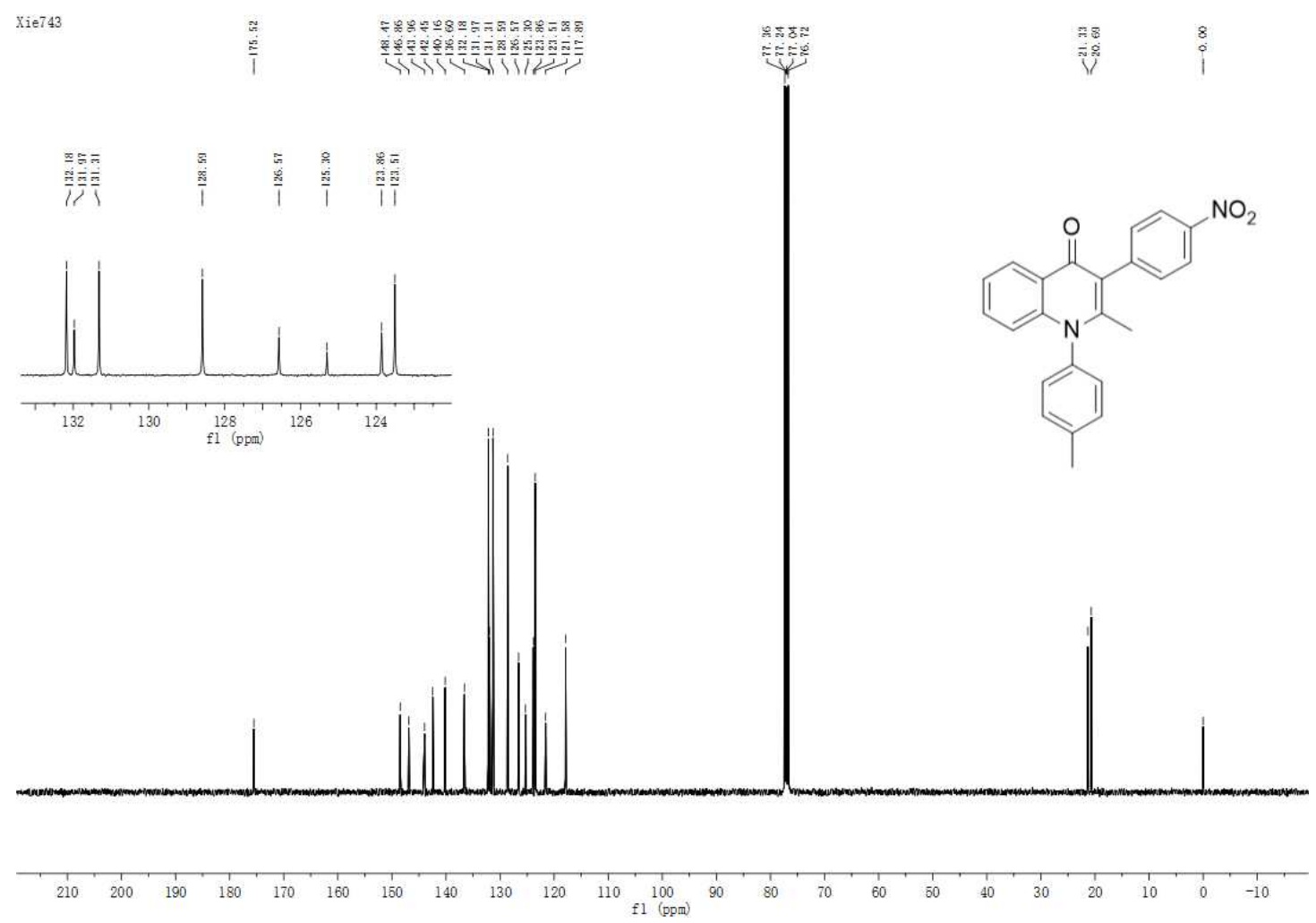

